\title{
Field-theoretical formulations of MOND-like gravity
}

\author{
Jean-Philippe Bruneton and Gilles Esposito-Farèse \\ $\mathcal{G} \mathbb{R} \varepsilon \mathbb{C O}$, Institut d'Astrophysique de Paris, \\ UMR 7095-CNRS, Université Pierre et Marie Curie-Paris6, \\ 98bis boulevard Arago, F-75014 Paris, France
}

(Dated: May 25, 2007)

\begin{abstract}
Modified Newtonian dynamics (MOND) is a possible way to explain the flat galaxy rotation curves without invoking the existence of dark matter. It is however quite difficult to predict such a phenomenology in a consistent field theory, free of instabilities and admitting a well-posed Cauchy problem. We examine critically various proposals of the literature, and underline their successes and failures both from the experimental and the field-theoretical viewpoints. We exhibit new difficulties in both cases, and point out the hidden fine tuning of some models. On the other hand, we show that several published no-go theorems are based on hypotheses which may be unnecessary, so that the space of possible models is a priori larger. We examine a new route to reproduce the MOND physics, in which the field equations are particularly simple outside matter. However, the analysis of the field equations within matter (a crucial point which is often forgotten in the literature) exhibits a deadly problem, namely that they do not remain always hyperbolic. Incidentally, we prove that the same theoretical framework provides a stable and well-posed model able to reproduce the Pioneer anomaly without spoiling any of the precision tests of general relativity. Our conclusion is that all MOND-like models proposed in the literature, including the new ones examined in this paper, present serious difficulties: Not only they are unnaturally fine tuned, but they also fail to reproduce some experimental facts or are unstable or inconsistent as field theories. However, some frameworks, notably the tensor-vector-scalar (TeVeS) one of Bekenstein and Sanders, seem more promising than others, and our discussion underlines in which directions one should try to improve them.
\end{abstract}

PACS numbers: 04.50.+h, 95.30.Sf, 95.35.+d 


\section{INTRODUCTION}

Although general relativity (GR) passes all precision tests with flying colors [1, 2], there remain some puzzling experimental issues, notably the fact that the Universe seems to be filled with about $72 \%$ of dark energy and $24 \%$ of dark matter, only $4 \%$ of its energy content being made of ordinary baryonic matter [3, 4]. Dark energy, a fluid whose negative pressure is close to the opposite of its energy density, would be responsible for the present accelerated expansion of the Universe [], [6, 7, [8]. Dark matter, a pressureless and noninteracting component of matter detected only by its gravitational influence, is suggested by several experimental data, notably the flat rotation curves of clusters and galaxies [9]. Another experimental issue is the anomalous extra acceleration $\delta a \approx 8.5 \times 10^{-10} \mathrm{~m} . \mathrm{s}^{-2}$ towards the Sun that the two Pioneer spacecrafts exhibited between 30 and 70 AU [10] (see also [11]).

Actually, none of these issues contradicts GR nor Newtonian gravity directly. Indeed, dark energy may be understood as the existence of a tiny cosmological constant $\Lambda \approx 3 \times$ $10^{-122} c^{3} /(\hbar G)$, or as a scalar field (called quintessence) slowly rolling down a potential [12, 13, 14]. Many candidates of dark matter particles have also been predicted by different theoretical models (notably the class of neutralinos, either light or more massive, occurring in supersymmetric theories; see e.g. [15]), and numerical simulations of structure formation have obtained great successes while incorporating such a dark matter (see, e.g., [16]). [It remains however to explain why $\Lambda$ is so small but nonzero, and why the dark energy and dark matter densities happen to be today of the same order of magnitude.] The Pioneer anomaly seems more problematic, but the two spacecrafts are identical and were not built to test gravity, therefore one must keep cautious in interpreting their data. A dedicated mission would be necessary to confirm the existence of such an anomalous acceleration.

Nevertheless, these various issues, considered simultaneously, give us a hint that Newton's law might need to be modified at large distances, instead of invoking the existence of several dark fluids. To avoid the dark matter hypothesis, Milgrom [17] proposed in 1983 such a phenomenological modification, which superbly accounts for galaxy rotation curves [18] (although galaxy clusters anyway require some amount of dark matter), and automatically recovers the Tully-Fisher law [19] $v_{\infty}^{4} \propto M$ (where $M$ denotes the baryonic mass of a galaxy, and $v_{\infty}$ the asymptotic circular velocity of visible matter in its outer region). The norm $a$ of a particle's acceleration is assumed to be given by its Newtonian value $a_{N}$ when it is greater than a universal constant $a_{0} \approx 1.2 \times 10^{-10} \mathrm{~m} \cdot \mathrm{s}^{-2}$, but to read $a=\sqrt{a_{N} a_{0}}$ in the small-acceleration regime $a<a_{0}$. In particular, the gravitational acceleration should now read $a=\sqrt{G M a_{0}} / r$ at large distances, instead of the usual $G M / r^{2}$ law.

Various attempts have been made to derive such a modified Newtonian dynamics (MOND) from a consistent relativistic field theory. The main aim of the present paper is to examine them critically, by underlining their generic difficulties and how some models managed to solve them. Three classes of difficulties may actually be distinguished: (i) theoretical ones, namely whether the proposed model derives from an action principle, is stable and admits a well-posed Cauchy problem; (ii) experimental ones, in particular whether solar-system and binary-pulsar tests are passed, and whether the predicted light defection by galaxies or haloes is consistent with weak-lensing observations; and (iii) "esthetical" ones, i.e., whether the proposed model is natural enough to be considered as predictive, or so finetuned that it almost becomes a fit of experimental data. These different classes of difficulties are anyway related, because fine-tuning is often necessary to avoid an experimental problem, and because some tunings sometimes hide serious theoretical inconsistencies. 
We will not address below the problem of dark energy, although it may also be tackled with a modified-gravity viewpoint. For instance the DGP brane model [20, 21] predicts an accelerated expansion of the Universe without invoking a cosmological constant [22, 23], and the k-essence models [24, 25, 26] also reproduce many cosmological features in a somewhat more natural way than quintessence theories. Some of our theoretical discussions below, notably about stability and causality, are nevertheless directly relevant to such models. Indeed, k-essence theories are characterized by a non-linear function of a scalar field's kinetic term, precisely in the same way relativistic aquadraric Lagrangians (RAQUAL) were devised to reproduce the MOND phenomenology in a consistent field theory [27]. Our aim is not to discuss the Pioneer anomaly in depth either. Although the numerical value of these spacecrafts' extra acceleration $\delta a$ is of the same order of magnitude as the MOND parameter $a_{0}$, there indeed exist important differences between their behavior and the MOND dynamics. However, we will come back to the Pioneer anomaly at the very end of the present paper. While analyzing a new class of models a priori devised to reproduce the MOND phenomenology, we will show that it can account for the Pioneer anomaly without spoiling any of the precision tests of general relativity, while being stable and admitting a well-posed Cauchy problem.

Our paper is organized as follows. In Sec. III, we examine the various ways one may try to modify the laws of gravity in a consistent relativistic field theory, and give a critical review of several models proposed in the literature. We notably underline that an action should not depend on the mass of a galaxy, otherwise one is defining a different theory for each galaxy. We also recall why higher-order gravity is generically unstable, contrary to scalar-tensor theories. When the dynamics of the scalar field is defined by an aquadratic kinetic term (RAQUAL or k-essence theories), we discuss the different consistency conditions it must satisfy. We point out that such conditions suffice for local causality to be satisfied, although some modes may propagate faster than light or gravitational waves. We finally recall how Ref. [27] reproduced the MOND gravitational force thanks to a RAQUAL model, but we exhibit a serious fine-tuning problem which does not seem to have been discussed before or at least was not pointed out so clearly.

In Sec. III, we recall that many models fail to reproduce the observed light deflection by "dark matter" haloes. We exhibit a counter-example to an erroneous claim in the literature, although this counter-example does not reproduce the correct MOND phenomenology. We also recall how a "disformal" (i.e., non-conformal) coupling of the scalar field to matter allowed Refs. [28, 29] to predict the right light deflection, but that this class of models has been discarded too quickly, because of the existence of superluminal gravitons. Actually, the consistency and causality of such models is clear when analyzed in the Einstein frame. On the other hand, we show that the consistency of the field equations within matter implies new conditions which must be satisfied by the functions defining the theory. As far as we are aware, these crucial conditions were not derived previously in the literature. We finally discuss how the best present model, called TeVeS [30, 31, 32, 33], solved the problem of light deflection by considering a stratified theory, involving a unit vector field in addition to a metric tensor and one or several scalar fields.

In Sec. IV, we discuss the various difficulties which anyway remain in this TeVeS model, including some which have already been discussed in the literature and that we merely summarize. Reference [34] proved that the TeVeS Hamiltonian is not bounded by below, and therefore that the model is unstable. We also point out other instabilities in related models. Many papers underlined that stratified theories define a preferred frame, and that 
they are a priori inconsistent with solar-system tests of local Lorentz invariance of gravity. However, the vector field is assumed to be dynamical in TeVeS, and it has been argued in [30, 31] that such tests are now passed. Although we do not perform a full analysis ourselves, our discussion suggests that preferred-frame effects are actually expected in TeVeS, and that they may be avoided only at the expense of an unnatural fine-tuning. The reason why disformal models were discarded in favor of the stratified TeVeS theory was mainly to avoid superluminal gravitons. However, the opposite phenomenon occurs in TeVeS: photons (and high-energy matter particles) propagate faster than gravitons. References [35, 36] actually proved that the observation of high-energy cosmic rays imposes tight constraints on such a behavior, because these rays should have lost their energy by Cerenkov radiation of gravitational waves. After recalling this argument, we conclude that the sign of one of the TeVeS parameters should be flipped, implying that gravitons now propagate faster than light, like in the RAQUAL models. We finally discuss briefly binary-pulsar constraints, still without performing a full analysis, but underlining that a large amount of energy should be emitted as dipolar waves of the scalar field. The matter-scalar coupling constant should thus be small enough in TeVeS to pass binary-pulsar tests, and this implies an unnatural fine-tuning of its Lagrangian.

Section $\nabla$ is devoted to new models whose point of view differs significantly from those of the literature. They are extremely simple in vacuum, reducing respectively to general relativity and to Brans-Dicke theory. The MOND phenomenology is obtained via a nonminimal coupling of matter to the gravitational field(s). However, the first model exhibits a subtle instability, similar to the one occurring in higher-order gravity (although no tachyon nor ghost degree of freedom may be identified in vacuum). The second model avoids this instability due to higher derivatives, and reproduces the MOND phenomenology (including light deflection) quite simply, as compared to the literature. However, our analysis of the field equations shows that they do not remain hyperbolic within the dilute gas in outer regions of a galaxy. Therefore, although promising, this model is inconsistent. But the same framework provides a simple model to reproduce consistently the Pioneer anomaly, without spoiling the precision tests of general relativity.

Finally, we present our conclusions in Sec. VI. We notably mention other experimental constraints that any complete MOND-like theory of gravity should also satisfy, besides those which are discussed in the present paper.

\section{LOOKING FOR MOND-LIKE FIELD THEORIES}

\section{A. General relativity}

Einstein's general relativity is based on two independent hypotheses, which are most conveniently described by decomposing its action as $S=S_{\text {gravity }}+S_{\text {matter }}$. First, it assumes that all matter fields, including gauge bosons, are minimally coupled to a single metric tensor, that we will denote $\tilde{g}_{\mu \nu}$ throughout the present paper. This metric defines the lengths and times measured by laboratory rods and clocks (made of matter), and is thereby called the "physical metric" (the name "Jordan metric" is also often used in the literature). The matter action may thus be written as $S_{\text {matter }}\left[\psi ; \tilde{g}_{\mu \nu}\right]$, where $\psi$ denotes globally all matter fields, and where the angular brackets indicate a functional dependence. This so-called "metric coupling" implies the weak equivalence principle, i.e., the fact that laboratory-size objects fall with the same acceleration in an external gravitational field, which is experimentally 
verified to a few parts in $10^{13}$ [37, 38]. For instance, the action of a point-particle reads $S_{\mathrm{pp}}=-\int m c^{2} \sqrt{-\tilde{g}_{\mu \nu}(x) v^{\mu} v^{\nu}} d t$, and depends both on the spacetime position $x^{\mu}$ of the particle and its velocity $v^{\mu} \equiv d x^{\mu} / d t$. The second building block of GR is the EinsteinHilbert action

$$
S_{\text {gravity }}=\frac{c^{4}}{16 \pi G} \int \frac{d^{4} x}{c} \sqrt{-g_{*}} R^{*},
$$

which defines the dynamics of a spin-2 field $g_{\mu \nu}^{*}$, called the "Einstein metric". We use the sign conventions of [39], notably the mostly-plus signature, and always indicate with a tilde or a star (either upper or lower) which metric is used to construct the corresponding quantity. For instance, $g_{*} \equiv \operatorname{det}\left(g_{\mu \nu}^{*}\right)$ is the determinant of the Einstein metric and $R^{*}$ its scalar curvature. Similarly, we will denote as $\tilde{\nabla}_{\mu}$ and $\nabla_{\mu}^{*}$ the covariant derivatives corresponding respectively to the Jordan and Einstein metrics, and as $\tilde{g}^{\mu \nu}$ and $g_{*}^{\mu \nu}$ the inverses of these two metrics. This rather heavy notation will allow us to be always sure of what we are talking about in the following. Einstein's second hypothesis is that both metrics coincide: $\tilde{g}_{\mu \nu}=g_{\mu \nu}^{*}$.

Milgrom analyzed in [40, 41] the consequences of modifying the matter action, that he called "modified inertia". He focused on a point particle in an external gravitational field, and assumed that its action could depend also on the acceleration $\mathbf{a} \equiv d \mathbf{v} / d t$ and its higher time-derivatives. However, he proved that to obtain both the Newtonian and the MOND limits and satisfy Galileo invariance, the action must depend on all time-derivatives $d^{n} \mathbf{v} / d t^{n}$ to any order, i.e., that the action is necessarily nonlocal. This does not necessarily violate causality (see the counter-example in [42]), and is actually a good feature for the stability of the theory (see Sec. IIC below), but the actual computation of the predictions is quite involved. We refer the reader to the detailed paper [40] for more information about this interesting viewpoint, but we focus below on metric field theories, i.e., such that matter is minimally coupled to $\tilde{g}_{\mu \nu}$ as in GR, with only first derivatives of the matter fields entering the action.

Another way to modify GR is to assume that this physical metric does not propagate as a pure spin-2 field, i.e., that its dynamics is no longer described by the Einstein-Hilbert action (2.1), and that $\tilde{g}_{\mu \nu}$ is actually a combination of various fields. Since the weak equivalence principle is very well tested experimentally, most MOND-like models in the literature focused on such a "modified gravity" viewpoint, and we will examine them in the present paper. The fact that they involve extra fields, besides the usual graviton (fluctuation of $g_{\mu \nu}^{*}$ ) and the various matter fields entering the Standard Model of particle physics, makes the distinction with dark matter models rather subtle. The crucial difference is that, in the dark-matter paradigm, the amount of dark matter is imposed by initial conditions, and its clustering generates gravitational wells in which baryonic matter fall to form galaxies and large-scale structures. On the other hand, in the modified-gravity viewpoint, baryonic matter generates itself an effective dark-matter halo $M_{\text {dark }} \propto \sqrt{M_{\text {baryon }}}$. Such a halo may just be an artifact of the way we interpret the gravitational field of baryonic matter alone at large distances. But it may also be a real dark-matter halo, made of the extra gravitational fields, and generating itself a Newtonian potential. In such a case, the difference with standard darkmatter models would be that its mass $M_{\text {dark }}$ is imposed as above by the baryonic one, and modified gravity could thus be considered as a constrained class of dark-matter models. Most of the theories that we will mention in the following predict that the energy density of the extra gravitational degrees of freedom is negligible with respect to baryonic matter: $\left|T_{\mu \nu}^{\text {extra fields }}\right| \ll\left|T_{\mu \nu}^{\text {matter }}\right|$. Therefore, the effective dark-matter haloes will be most of the time 
artifacts of our interpretation of observations. However, we will also consider in Sec. IIIB a model of the "constrained dark matter" type $\left(\left|T_{\mu \nu}^{\text {extra fields }}\right| \gg\left|T_{\mu \nu}^{\text {matter }}\right|\right)$, as a counter-example to some claims in the literature about light deflection. ${ }^{1}$

As suggested by Milgrom, one may also consider models modifying both inertia and gravity. Paradoxically, as we will see in Sec. VA below, there also exists a non-trivial (nonGR) possibility in which neither of them is modified in the above sense. Matter will be universally coupled to a second-rank symmetric tensor $\tilde{g}_{\mu \nu}$, i.e., still described by an action $S_{\text {matter }}\left[\psi ; \tilde{g}_{\mu \nu}\right]$, and the dynamics of gravity will be described by the pure Einstein-Hilbert action (2.1) in vacuum, but the two metrics $\tilde{g}_{\mu \nu}$ and $g_{\mu \nu}^{*}$ will nevertheless differ within matter.

\section{B. Various ideas in the literature}

Although interesting from a phenomenological point of view, some models proposed in the literature write field equations which do not derive from an action, and cannot be obtained within a consistent field theory. For instance, actually not to reproduce a MONDlike behavior but anyway as a model of dark matter, Ref. [45] proposes to couple differently dark matter and baryonic matter to gravity (an idea explored in the cosmological context by various authors, notably [46, 47, 48, 49, 50, 51, 52, 53]). The authors underline themselves that it cannot be considered as a fundamental theory (and they actually need some negative energy to obtain a repulsive force), but it is also instructive to stress that it cannot derive from an action. Indeed, the scalar field entering this model is assumed not to be generated by baryons, which implies that the scalar-baryon vertex vanishes. On the other hand, the assumed equation of motion for this baryonic matter does depend on the scalar field, thereby implying that the scalar-baryon vertex does not vanish. In conclusion, although the behavior of such a model might be mimicked as an effective theory of a more fundamental one, it cannot be described itself as a field theory, and notably does not satisfy conservation laws. In the present paper, our discussion will be restricted to consistent field theories.

Some models in the literature do write actions, but they depend on the (baryonic or luminous) mass $M$ of the galaxy [54, 55]. For instance Ref. [55] proposes a gravitational action in which the kinetic term is some power of the Ricci scalar $R^{1-n / 2}$. Since this implies, in general, an asymptotic velocity of the form $v^{2} \propto n c^{2}+\mathcal{O}(G M / r)$, Ref. [55] then proposes to replace $n$ by $\sqrt{G M a_{0} / c^{4}}$ in order to recover the Tully-Fisher law. However, without an additional prescription (which must be non-local in nature) specifying which mass $M$ enters the action, this theory is ill defined. We will come back to such $f(R)$ theories of gravity in Sec. IIC and in Sec. IID, It should be also underlined that a gravitational force $\propto 1 / r$ is not difficult to obtain by choosing an appropriate scalar field potential, for instance, as we will illustrate in Sec. IID below. However, the second crucial feature of MOND, which arises from the Tully-Fisher law, is also the factor $\sqrt{G M a_{0}} \propto \sqrt{M}$ multiplying this $1 / r$. Some consistent field theories actually obtain a flattening of rotation curves but do not predict the right amplitude of the asymptotic velocity [56, 57].

In [58, 59], Einstein and Straus studied an extension of general relativity in which the metric tensor is nonsymmetric, $g_{\mu \nu} \neq g_{\nu \mu}$. In modern language, this corresponds to considering

\footnotetext{
${ }^{1}$ Let us also mention that the recent reinterpretation of MOND proposed in Refs. [43, 44] is also of the constrained dark matter type: The MOND force $\propto 1 / r$ is caused by a background of gravitational dipoles contributing directly to the Newtonian potential.
} 
an antisymmetric tensor field $B_{[\mu \nu]}$ as a partner to the usual graviton $g_{(\mu \nu)}$. Moffat analyzed the phenomenology of this class of models in many papers (starting with [60]), and it was shown to define a consistent and stable field theory provided $B_{[\mu \nu]}$ is massive [61]. More recently, Moffat showed that it may reproduce the flat rotation curves of clusters and galaxies [62, 63, 64, 65, 66], probably even better that the original MOND proposal. However, several assumptions are needed to obtain such a prediction, and it cannot yet be considered as a predictive field theory, in our opinion. Indeed, the author points out himself that a constant entering his action must take three different values at the solar system, galaxy, and cluster scales. It must therefore be considered as a running (renormalized) coupling constant instead of a pure number imposed in the action, and a complete theory should be able to predict the three needed values. A related difficulty is the fact that the force generated by the antisymmetric tensor field $B_{[\mu \nu]}$ is repulsive, whereas galaxy rotation curves need a gravitational attraction greater that the Newtonian one. Here again, the author invokes renormalization of the constants to obtain the right behavior, but does not provide a full derivation. But the most instructive difficulty of Ref. [63] is that the extra force is predicted to be $\propto k M^{2} / r$ instead of the needed $\propto \sqrt{M} / r$ behavior. The author first assumed in [63] that an unknown mechanism could tune the proportionality constant to be $k \propto M^{-3 / 2}$, so that the right MOND phenomenology would be recovered. More recently [65], he managed to derive this relation by assuming a particular form of a potential. However, this potential does depend explicitly on the mass $M$ of the galaxy, and the predictivity of the model is thus lost. In conclusion, although the framework of nonsymmetric gravity seems promising, it is not yet formulated as a closed consistent field theory, and it should only be considered at present as a phenomenological fit of observational data.

In the following, we will focus on field theories whose actions do not depend on the mass or scale of the considered objects. They may involve fine-tuned numbers, but they will be fixed once for all. This is necessary to define a predictive model, but we will underline that it does not suffice to prove its consistency. In particular, the stability needs to be analyzed carefully.

\section{Higher-order gravity}

One-loop divergences of quantized GR [67] are well known to generate terms proportional to $^{2} R^{2}, R_{\mu \nu}^{2}$ and $R_{\mu \nu \rho \sigma}^{2}$. It is thus natural to consider extensions of GR already involving such higher powers of the curvature tensor in the classical action. At quadratic order, it is always possible to write them as

$$
S_{\text {gravity }}=\frac{c^{4}}{16 \pi G} \int \frac{d^{4} x}{c} \sqrt{-g}\left[R+\alpha C_{\mu \nu \rho \sigma}^{2}+\beta R^{2}+\gamma \mathrm{GB}\right]
$$

where $C_{\mu \nu \rho \sigma}$ denotes the Weyl (fully traceless) tensor, GB $\equiv R_{\mu \nu \rho \sigma}^{2}-4 R_{\mu \nu}^{2}+R^{2}$ is the Gauss-Bonnet topological invariant, and $\alpha, \beta, \gamma$ are constants having the dimension of a length squared (i.e., $(\hbar / m c)^{2}$ if $m$ denotes the corresponding mass scale). The topological invariant $\mathrm{GB}$ does not contribute to the local field equations and may thus be discarded. In his famous thesis [68], Stelle proved that such an action gives a renormalizable quantum

\footnotetext{
${ }^{2}$ To simplify, we do not write the tildes which should decorate all quantities in this subsection, indicating that $g_{\mu \nu}=\tilde{g}_{\mu \nu}$ will be later assumed to be minimally coupled to matter.
} 
theory, to all orders, provided both $\alpha$ and $\beta$ are nonzero. However, he also underlined that this cannot be the ultimate answer to quantum gravity because such a theory contains a ghost, i.e., a degree of freedom whose kinetic energy is negative. To understand this intuitively, it suffices to decompose the schematic propagator in irreducible fractions:

$$
\frac{1}{p^{2}+\alpha p^{4}}=\frac{1}{p^{2}}-\frac{1}{p^{2}+1 / \alpha}
$$

where the first term, $1 / p^{2}$, corresponds to the propagator of the usual massless graviton, whereas the second term corresponds to a massive degree of freedom such that $m^{2}=1 / \alpha$. The negative sign of this second term indicates that it carries negative energy. Note that one cannot change its sign by playing with that of $\alpha$ : If $\alpha<0$, this extra degree of freedom is even a tachyon (negative mass squared), but it anyway remains a ghost (negative kinetic energy). The problem with such a ghost degree of freedom is that the theory is violently unstable. Indeed, the vacuum can disintegrate in an arbitrary amount of positive-energy usual gravitons whose energy is balanced by negative-energy ghosts. Even at the classical level, although such an instability is difficult to exhibit explicitly on a toy model, one expects anyway the perturbations of a given background to generically diverge, by creating growing gravitational waves containing both positive-energy (massless) modes and negative-energy (massive) ones.

The actual calculation, taking into account all contracted indices in the propagators, confirms the above schematic reasoning for the $\alpha C_{\mu \nu \rho \sigma}^{2}$ term in action (2.2), and shows that the extra massive mode has also a spin 2, like the usual massless graviton. On the other hand, the $\beta R^{2}$ term generates in fact a positive-energy massive scalar degree of freedom. We will recall a well-known and simple derivation in Sec. IID below. The intuitive explanation is that the scalar mode corresponds in GR to the negative gravitational binding energy (which is not an actual degree of freedom because it is constrained by the field equations), so that the negative sign entering the right-hand side of Eq. (2.3) in fact multiplies an already negative term. The resulting extra degree of freedom thereby appears as a positive-energy scalar field [69].

More general actions $f\left(R, R_{\mu \nu}, R_{\mu \nu \rho \sigma}\right)$ have been considered several times in the literature, notably in [70] and [71]. The conclusion is that they generically contain the massive spin-2 ghost exhibited above, which ruins the stability of the theory. The only allowed models, within this class, are functions of the scalar curvature alone, $f(R)$, that we will study in Sec. IID below.

The fact that such functions of the curvature generically involve a ghost tells us that some particular models may avoid it. For instance, Ref. [72] considers functions whose quadratic term reduces to the Gauss-Bonnet topological invariant $\mathrm{GB} \equiv R_{\mu \nu \rho \sigma}^{2}-4 R_{\mu \nu}^{2}+R^{2}$. Reference [73. even focuses on a Lagrangian of the form $R+f(\mathrm{~GB})$, whose quadratic term is again the Gauss-Bonnet invariant. Around a flat Minkowski background, the only quadratic kinetic term $\mathcal{O}\left(g_{\mu \nu}-\eta_{\mu \nu}\right)^{2}$ is thus the one coming from the standard Einstein-Hilbert term $R$, so that no propagator can be defined for any ghost degree of freedom. However, Ref. [70] underlined that flat spacetime is generically not a solution of such higher-order gravity theories. Around a curved background, the second-order expansion of higher-order terms like $\left(R_{\mu \nu \rho \sigma}^{2}\right)^{n}$ thereby generates a nonzero kinetic term for the spin-2 ghost (see Fig. 6] in Sec. VA below for a diagrammatic illustration).

One may still try to devise higher-order gravity models such that their second-order expansion around any background never generates a negative-energy kinetic term. However, 
deadly instabilities still exist in such theories, even if no ghost degree of freedom can be identified in a perturbative way. Indeed, as recalled notably in [69], their Hamiltonian is generically non-bounded by below, because it is linear in at least one of the canonical momenta. As in [69], let us illustrate this on the simplest example of a Lagrangian depending on a variable $q$ and its first two time derivatives, say $\mathcal{L}(q, \dot{q}, \ddot{q})$. If $\ddot{q}$ can be eliminated from $\mathcal{L}$ by partial integration, we are in the standard case of a theory depending only on first derivatives, and we know that some models do give a Hamiltonian which is bounded by below. This is the case when $\ddot{q}$ appears only linearly in $\mathcal{L}$, even multiplied by a function of $q$ and $\dot{q}$ since $\int d t q^{n} \dot{q}^{m} \ddot{q}=-n /(m+1) \int d t q^{n-1} \dot{q}^{m+2}+$ boundary terms. Let us thus consider only the case of a "non-degenerate" Lagrangian, i.e., such that the definition $p_{2} \equiv \partial \mathcal{L} / \partial \ddot{q}$ can be inverted to express $\ddot{q}$ as a function of $q, \dot{q}$ and $p_{2}$ :

$$
\ddot{q}=f\left(q, \dot{q}, p_{2}\right) .
$$

In such a case, Ostrogradski showed in 1850 [74] that the initial data must be specified by two pairs of conjugate momenta, that he defined as

$$
\begin{array}{ll}
q_{1} \equiv q, & p_{1} \equiv \frac{\partial \mathcal{L}}{\partial \dot{q}}-\frac{d}{d t}\left(\frac{\partial \mathcal{L}}{\partial \ddot{q}}\right), \\
q_{2} \equiv \dot{q}, & p_{2} \equiv \frac{\partial \mathcal{L}}{\partial \ddot{q}},
\end{array}
$$

and he proved that the following definition of the Hamiltonian

$$
\mathcal{H} \equiv p_{1} \dot{q}_{1}+p_{2} \dot{q}_{2}-\mathcal{L}(q, \dot{q}, \ddot{q})
$$

does generate time translations. Indeed, $\dot{q}_{i}=\partial \mathcal{H} / \partial p_{i}$ and $\dot{p}_{i}=-\partial \mathcal{H} / \partial q_{i}$ reproduce the Euler-Lagrange equations of motion deriving from the original Lagrangian $\mathcal{L}$. However, this Hamiltonian must be expressed in terms of the momenta $q_{i}$ and $p_{i}$ defined in Eqs. (2.5) above. Recalling that Eq. (2.4) allows us to write $\ddot{q}=f\left(q_{1}, q_{2}, p_{2}\right)$, one gets

$$
\mathcal{H}=p_{1} q_{2}+p_{2} f\left(q_{1}, q_{2}, p_{2}\right)-\mathcal{L}\left(q_{1}, q_{2}, f\left(q_{1}, q_{2}, p_{2}\right)\right)
$$

The crucial problem of this expression is that it is linear in $p_{1}$, and therefore non-bounded by below [69]. In other words, the theory is necessarily unstable, even if one does not identify any explicit contribution $-p_{i}^{2}$ defining a ghost degree of freedom.

This result can be straightforwardly extended to (non-degenerate) models depending on even higher time-derivatives of $q$, say up to $d^{n} q / d t^{n}$. In that case, Ref. [74] shows that the Hamiltonian is linear in $n-1$ of the momenta $p_{i}$, and thereby non-bounded by below. On the other hand, Ostrogradski's construction of the Hamiltonian cannot be used if $\mathcal{L}$ depends on an infinite number of time derivatives, i.e., if it defines a nonlocal theory. In such a case, the theory may actually be stable although its expansion looks pathological [75]. This is notably the case in the effective low-energy models defined by string theory, which do involve quadratic curvature terms like Eq. (2.2) above, but also any higher derivative of the curvature tensor (whose phenomenological effects occur at the same order as the quadratic terms). Nonlocal models of MOND have been studied in the literature [42], and they can be proved to satisfy anyway causality, but they remain difficult to study from a phenomenological point of view. In the following, we will focus on local field theories. 


\section{Scalar-tensor theories}

As mentioned in the previous section, gravity models whose Lagrangians are given by functions of the scalar curvature, $f(R)$, do not exhibit any ghost degree of freedom, and avoid the generic "Ostrogradskian" instability of higher-derivative theories [69]. Let us recall here the simplest way to prove it (see [70, 71, 76, 77, 78, 79, 80, 81, 82], and note that the following derivation assumes that the scalar curvature $R$ is a function of the metric $g_{\mu \nu}$ and its derivatives alone; the derivation and the result differ in the first-order Palatini formalism, where the scalar field does not acquire any kinetic term [83, 84]). We start from an action

$$
S_{\text {gravity }}=\int d^{4} x \sqrt{-\tilde{g}} f(\tilde{R}),
$$

where the global factor $c^{3} / 16 \pi G$ is temporarily set to 1 , to simplify. We now introduce a Lagrange parameter $\phi$ to rewrite this action as

$$
S_{\text {gravity }}=\int d^{4} x \sqrt{-\tilde{g}}\left\{f(\phi)+(\tilde{R}-\phi) f^{\prime}(\phi)\right\} .
$$

The field equation for $\phi$ reads $(\tilde{R}-\phi) f^{\prime \prime}(\phi)=0$, and implies $\phi=\tilde{R}$ within each spacetime domain where $f^{\prime \prime}(\phi) \neq 0$. [If there exist hypersurfaces or more general domains where $f^{\prime \prime}(\phi)=0$, then either $f$ is constant and there is no gravitational degree of freedom in such domains, or $f(\tilde{R}) \propto \tilde{R}-2 \Lambda$ and the theory reduces to GR plus a possible cosmological constant. In both cases, the scalar degree of freedom that we will exhibit below cannot propagate within such domains. A consistent field theory, without any discontinuity of its degrees of freedom, can thus be defined only within the domains, possibly infinite, where $f^{\prime \prime}(\tilde{R}) \neq 0$.] The field equations for the metric $\tilde{g}_{\mu \nu}$ may now be derived from action (2.9), and one finds that they reduce to those deriving from action (2.8) when $\phi$ is replaced by $\tilde{R}$. This can also be seen by the a priori illicit use of the field equation $\phi=\tilde{R}$ directly within action (2.9), which reduces trivially to (2.8). In other words, the theory defined by action (2.8) is equivalent to the scalar-tensor one

$$
S_{\text {gravity }}=\int d^{4} x \sqrt{-\tilde{g}}\left\{f^{\prime}(\phi) \tilde{R}-0\left(\partial_{\mu} \phi\right)^{2}-\left[\phi f^{\prime}(\phi)-f(\phi)\right]\right\}
$$

within each spacetime domain where $f^{\prime \prime}(\tilde{R})$ never vanishes. If one redefines the scalar field as $\Phi \equiv f^{\prime}(\phi)$, this action takes the form of the famous (Jordan-Fierz)-Brans-Dicke theory [85, 86, 87] with no explicit kinetic term for $\Phi$, i.e., with a vanishing $\omega_{\mathrm{BD}}$ parameter, and with a potential defined by the last term within square brackets. It should be stressed that solar-system experiments [88] impose the bound $\omega_{\mathrm{BD}}>4000$ when the scalar-field potential vanishes. Therefore, if matter is assumed to be coupled to $\tilde{g}_{\mu \nu}$, the resulting scalar degree of freedom needs to be massive enough to have a negligible effect in the solarsystem gravitational physics. The shape of the initial function $f(\tilde{R})$ is thus constrained by experiment.

Although action (2.10) does not involve any explicit kinetic term for $\phi$ (or the Brans-Dicke scalar $\Phi)$, this scalar field does propagate. Indeed, the first contribution $f^{\prime}(\phi) \tilde{R}$ contains terms of the form $\phi \partial^{2} \tilde{g}$, i.e., cross terms $-\partial \phi \partial \tilde{g}$ after partial integration. A redefinition of the fields then allows us to diagonalize their kinetic terms. This is achieved with the new 
variables

$$
\begin{aligned}
g_{\mu \nu}^{*} & \equiv f^{\prime}(\phi) \tilde{g}_{\mu \nu}, \\
\varphi & \equiv \frac{\sqrt{3}}{2} \ln f^{\prime}(\phi), \\
V(\varphi) & \equiv \frac{\phi f^{\prime}(\phi)-f(\phi)}{4 f^{\prime 2}(\phi)},
\end{aligned}
$$

and the full action, Eq. (2.10) plus the matter part $S_{\text {matter }}\left[\psi ; \tilde{g}_{\mu \nu}\right]$, now reads

$$
S=\frac{c^{4}}{4 \pi G} \int \frac{d^{4} x}{c} \sqrt{-g_{*}}\left\{\frac{R^{*}}{4}-\frac{1}{2} g_{*}^{\mu \nu} \partial_{\mu} \varphi \partial_{\nu} \varphi-V(\varphi)\right\}+S_{\text {matter }}\left[\psi ; \tilde{g}_{\mu \nu}=A^{2}(\varphi) g_{\mu \nu}^{*}\right]
$$

where we have put back the global factor $c^{3} / 16 \pi G$ multiplying the gravitational action, and where $A(\varphi)=e^{\varphi / \sqrt{3}}$. This is a particular case of scalar-tensor theory [89], where the precise matter-scalar coupling function $A(\varphi)=e^{\varphi / \sqrt{3}}$ comes from our initial hypothesis of a $f(\tilde{R})$ theory. More general models may involve an arbitrary nonvanishing function $A(\varphi)$ relating the Einstein metric $g_{\mu \nu}^{*}$ (whose fluctuations define the spin-2 degree of freedom) to the physical one $\tilde{g}_{\mu \nu}=A^{2}(\varphi) g_{\mu \nu}^{*}$. Action (2.12) clearly shows that the spin-0 degree of freedom $\varphi$ does propagate and carries positive kinetic energy. Of course, this does not suffice to guarantee the stability of the theory: The potential $V(\varphi)$, Eq. (2.11c), also needs to be bounded by below, and this imposes constraints on the initial function $f(\tilde{R})$.

Although such tensor-mono-scalar models are perfectly well-defined field theories, they do not reproduce the MOND phenomenology, at least not in the most natural cases. Indeed, when the potential $V(\varphi)$ has a negligible influence, the scalar-field contribution to the gravitational interaction is proportional to the Newtonian one, at lowest order, i.e., of the form $\varphi \propto G M / r c^{2}$ where $M$ is the baryonic mass of the body generating the field. Therefore, it does not give the MOND potential $\sqrt{G M a_{0}} \ln r$ we are looking for. It has been shown in [90, 91, 92] that Gaussian matter-scalar coupling functions $A^{2}(\varphi)=e^{\beta_{0} \varphi^{2}}$, with $\beta_{0}<0$, generate nonperturbative effects such that $\varphi$ is no longer strictly proportional to $M$, but still almost so for two different mass ranges. Therefore, the factor $\sqrt{M}$ we are looking for cannot be obtained that way either, besides the fact that the radial dependence of the scalar field is still $\propto 1 / r$ instead of being logarithmic. When the potential $V(\varphi)$ has a minimum, its second derivative defines the mass $m$ of the scalar degree of freedom, and its contribution to the gravitational interaction is of the Yukawa type, $\varphi \propto G M e^{-m r} / r c^{2}$, still not of the MONDian form, notably because its global factor is $M$ instead of $\sqrt{M}$. [Note that the Yukawa force is proportional to $\partial_{r} \varphi \propto G M e^{-m r}\left(1 / r^{2}+m / r\right)$, and therefore includes a MOND-like $1 / r$ contribution. However, it dominates the main $1 / r^{2}$ term only for $m r \gg 1$, i.e., precisely when the exponential $e^{-m r}$ makes the whole force quickly tend towards zero.] A "quintessence"-like potential, whose minimum occurs at $\varphi \rightarrow \infty$, seems better because it allows us to build a logarithmic gravitational potential. Indeed, if $V(\varphi)=-2 a^{2} e^{-b \varphi}$, where $a$ and $b$ are two constants, then $\varphi=(2 / b) \ln (a b r)$ is a solution of the vacuum field equation $\Delta \varphi=V^{\prime}(\varphi)$. However, not only this potential is unbounded by below, thereby spoiling the stability of the model, but $\varphi$ is also multiplied by a constant $2 / b$ (independent of the matter source) instead of being proportional to $\sqrt{M}$.

A generalization of $f(R)$ theories has been considered in Refs. [82, 93], where the Lagrangian density is given by some function of the scalar curvature and its iterated 
d'Alembertian, $f\left(R, \square R, \ldots, \square^{n} R\right)$. The theory can be shown to be generically equivalent to a tensor-multi-scalar theory [89] involving $n+1$ scalar fields. The signs of their respective kinetic terms depends on the numerical constants entering the function $f\left(R, \square R, \ldots, \square^{n} R\right)$, and there is thus no guarantee that they all carry positive energy in the general case. However, well chosen functions $f$ do define stable tensor-multi-scalar theories.

Although it seems difficult to reproduce MOND dynamics with tensor-mono-scalar models, general tensor-multi-scalar theories might actually succeed. Indeed a particular tensorbi-scalar model, called "phase coupling gravity" (PCG), has been shown to reproduce the Tully-Fisher law in some appropriate regime (there exists a critical mass above which MOND phenomenology arises) [94, 95]. However this model is marginally ruled out experimentally [30], and moreover needs a potential which is unbounded by below, thereby spoiling the stability of the model. This kind of theory has then been promoted to a "tensor-vector-biscalar" model [33], that we shall examine in more detail in Sec. IVA.

Among stable scalar-tensor theories, let us finally mention those involving a coupling of the scalar field to the Gauss-Bonnet topological invariant, i.e., involving a term of the form $f(\varphi) \times$ GB in the gravitational action. [Note that the function of $\varphi$ is a priori free, but that the Gauss-Bonnet term must appear linearly.] Contrary to the higher-order theories discussed in Sec. ПC above, such models do not contain ghost degrees of freedom. They have been studied in various contexts (see e.g. [96, 97, 98, 99, 100]), but not with the aim of reproducing the MOND phenomenology. Our own investigations showed that it is a priori

promising, because the Riemann tensor $\tilde{R}_{\mu \nu \rho \sigma}$ generated by a body of baryonic mass $M$ does not vanish outside it, and therefore gives us a local access to $M$. However, a second information is also needed to separate $M$ from the radial dependence, and we did not find any simple way to generate a potential $\sqrt{G M a_{0}} \ln r$. [It is actually possible if one defines a nonstandard kinetic term for the scalar field, as in Sec. IIE below, but the scalar-GaussBonnet coupling is not necessary in such a case.] A related but much simpler idea is explored in Sec. $\nabla$ below.

\section{E. K-essence or RAQUAL models}

The above scalar-tensor models may involve three functions of the scalar field, namely the potential $V(\varphi)$, the matter-scalar coupling function $A(\varphi)$, and the possible scalar-GaussBonnet coupling $f(\varphi) \times$ GB. Their generalization to $n$ scalar fields $\varphi^{a}$ [89] may also involve a $n \times n$ symmetric matrix $\gamma_{a b}\left(\varphi^{c}\right)$ depending on them and defining their kinetic term $g_{*}^{\mu \nu} \gamma_{a b}\left(\varphi^{c}\right) \partial_{\mu} \varphi^{a} \partial_{\nu} \varphi^{b}$. Their phenomenology is however similar to the single scalar case, at least if all of them carry positive energy and their potential is bounded by below, to ensure the stability of the theory [89]. On the other hand, a significantly different physics arises if one considers more general kinetic terms of the form $f(s, \varphi)$, where $s \equiv g_{*}^{\mu \nu} \partial_{\mu} \varphi \partial_{\nu} \varphi$ is the standard one. [Beware that the literature often uses the notation $X \equiv s / 2$, which actually does not change the following discussion.] Such models, without any matter-scalar coupling, have been studied in the cosmological context under the name of "k-inflation" [24] or "k-essence" [25, 26], the letter k meaning that their dynamics is kinetic dominated, as opposed to quintessence models in which the potential $V(\varphi)$ plays a crucial role. When a matter-scalar coupling $A(\varphi)$ is also assumed, such models have been studied to reproduce the MOND dynamics under the name of "Relativistic AQUAdratic Lagrangians" (RAQUAL) 
[27]. Their action is thus of the form

$$
S=\frac{c^{4}}{4 \pi G} \int \frac{d^{4} x}{c} \sqrt{-g_{*}}\left\{\frac{R^{*}}{4}-\frac{1}{2} f(s, \varphi)-V(\varphi)\right\}+S_{\text {matter }}\left[\psi ; \tilde{g}_{\mu \nu}=A^{2}(\varphi) g_{\mu \nu}^{*}\right],
$$

with $s \equiv g_{*}^{\mu \nu} \partial_{\mu} \varphi \partial_{\nu} \varphi$, and they can be considered as natural generalizations of the simplest $f(\tilde{R})$ model, Eq. (2.12). Note that the potential $V(\varphi)$ may be reabsorbed within the general function $f(s, \varphi)$, and that it is therefore unnecessary. However, since one often considers functions $f(s)$ of the kinetic term alone, it remains convenient to keep an explicit potential $V(\varphi)$. Reference [27] proved that an appropriate nonlinear function $f(s, \varphi)$ allows us to reproduce the MOND gravitational potential $\sqrt{G M a_{0}} \ln r$, but that some difficulties remain, as discussed below and in Secs. IIF and III.

Several conditions must be imposed on this function $f(s, \varphi)$ to guarantee the consistency of the field theory. For any real value of $s \in[-\infty,+\infty]$, one must have

(a) $f^{\prime}(s, \varphi)>0$,

(b) $2 s f^{\prime \prime}(s, \varphi)+f^{\prime}(s, \varphi)>0$,

where a prime denotes derivation with respect to $s$, i.e., $f^{\prime}=\partial f(s, \varphi) / \partial s$. The condition $f^{\prime}(s) \geq 0$, implied by (a), is necessary for the Hamiltonian to be bounded by below. Indeed, up to a global factor $c^{4} / 8 \pi G$ that we do not write to simplify the discussion, the contribution of the scalar field to this Hamiltonian reads $H=2\left(\partial_{0} \varphi\right)^{2} f^{\prime}(s)+f(s)$ in a locally inertial frame. If there existed a value $s_{-}$such that $f^{\prime}\left(s_{-}\right)<0$, then $H$ could be made arbitrarily large and negative by choosing diverging values of $\left(\partial_{0} \varphi\right)^{2}$ and $\left(\partial_{i} \varphi\right)^{2}$ such that $-\left(\partial_{0} \varphi\right)^{2}+$ $\left(\partial_{i} \varphi\right)^{2}=s_{-}$. On the other hand, conditions (a) and (b) are necessary and sufficient for the field equation to be always hyperbolic, so that the Cauchy problem is well posed for the scalar field. Indeed, it reads

$$
G^{\mu \nu} \nabla_{\mu}^{*} \nabla_{\nu}^{*} \varphi=\frac{1}{2} \frac{\partial f}{\partial \varphi}-s \frac{\partial f^{\prime}}{\partial \varphi}+\frac{\partial V}{\partial \varphi}-\frac{4 \pi G}{c^{3} \sqrt{-g_{*}}} \frac{\delta S_{\text {matter }}}{\delta \varphi},
$$

where $G^{\mu \nu} \equiv f^{\prime} g_{*}^{\mu \nu}+2 f^{\prime \prime} \nabla_{*}^{\mu} \varphi \nabla_{*}^{\nu} \varphi$ plays the role of an effective metric in which $\varphi$ propagates. The lowest eigenvalue of $G^{\mu \nu}$ is negative (i.e., defining a consistent time) only if condition (b) is satisfied, and it is easy to check $^{3}$ that the three others are then positive (defining spatial dimensions) if (a) is also satisfied. ${ }^{4}$ As a quick check of condition (b), one may for instance consider the particular case of a homogeneous scalar field $\left(\partial_{i} \varphi=0\right)$ in a locally inertial frame $\left(g_{\mu \nu}^{*}=\eta_{\mu \nu}\right)$ : The fact that we need $G^{00}=-f^{\prime}+2 f^{\prime \prime} \times(-s)<0$ then immediately yields inequality (b). Finally, conditions (a) and (b) together suffice for the

3 The simplest calculation consist in diagonalizing the matrix $G^{\mu \rho} g_{\rho \nu}^{*}$, instead of the tensor $G^{\mu \nu}$, and impose that its four eigenvalues are positive. This also ensures that there exists a coordinate system in which the time directions defined by $G_{\mu \nu}$ and $g_{\mu \nu}^{*}$ coincide.

4 One could also consider the degenerate case where $f^{\prime}(s)$ vanishes on some hypersurfaces. On them, the effective metric can then be put in the form $\operatorname{diag}(-1,0,0,0)$ in some appropriate basis, and thus define a consistent time, if and only if $f^{\prime \prime}(s)<0$. Strictly speaking, the scalar field equation is not hyperbolic in that case but it has nevertheless a well-posed Cauchy problem. Using condition (b), we can conclude that $f^{\prime}(s)$ may vanish only at negative values of $s$. 
Hamiltonian to be bounded by below, provided the function $f(s, \varphi)$ is analytic and $f(s=$ $0, \varphi)$ is itself bounded by below for any $\varphi$. Indeed, still in a locally inertial frame, we know that $s=-\left(\partial_{0} \varphi\right)^{2}+\left(\partial_{i} \varphi\right)^{2} \geq-\left(\partial_{0} \varphi\right)^{2}$. Together with condition (a), we can thus conclude that the Hamiltonian $H=2\left(\partial_{0} \varphi\right)^{2} f^{\prime}+f$ is greater than $-2 s f^{\prime}+f$, which is known to be a decreasing function of $s$ because of condition (b). Therefore, for any $s \leq 0$, the Hamiltonian is necessarily greater than $f(s=0, \varphi)$. On the other hand, for $s \geq 0$, we also know that $H \geq f \geq f(s=0, \varphi)$, first because $\left(\partial_{0} \varphi\right)^{2} \geq 0$ and then because condition (a) implies that $f$ is an increasing function of $s$. Although we used the hyperbolicity condition (b) to derive that the Hamiltonian is bounded by below, note that condition (b) is not implied by this boundedness, on the contrary. Nevertheless, it reappears when considering the propagation velocity of perturbations around a background [24], $c_{s}^{2}=f^{\prime} /\left(2 s f^{\prime \prime}+f^{\prime}\right)$. Such perturbations are unstable if $c_{s}^{2}<0$, and one may thus think that condition (b) should be implied by the boundedness by below of the Hamiltonian. However, the energy of the perturbations is only part of the total Hamiltonian, which also includes the energies of the background and its interactions with the perturbations. A total Hamiltonian bounded by below therefore does not suffice to guarantee the stability of perturbations; the hyperbolicity of the field equations, condition (b), is also crucial.

In Ref. [101] (see also [102]), a third condition was underlined for such k-essence models, besides (a) and (b) above:

(c) $f^{\prime \prime}(s) \leq 0$.

This extra condition is necessary for the causal cone of the scalar field to remain inside the light cone defined by the spacetime metric $g_{\mu \nu}^{*}$, i.e., to avoid superluminal propagation. The simplest way to derive this inequality is to consider a null vector $k^{\mu}$ with respect to the metric $G_{\mu \nu}=\left(1 / f^{\prime}\right)\left[g_{\mu \nu}^{*}-2 f^{\prime \prime} \partial_{\mu} \varphi \partial_{\nu} \varphi /\left(2 s f^{\prime \prime}+f^{\prime}\right)\right]$, defined as the inverse of the effective metric $G^{\mu \nu}$ entering Eq. (2.14) above. [We assume here that $f^{\prime}$ never vanishes.] The equation $G_{\mu \nu} k^{\mu} k^{\nu}=0$ then gives $g_{\mu \nu}^{*} k^{\mu} k^{\nu}=2 f^{\prime \prime}\left(k^{\mu} \partial_{\mu} \varphi\right)^{2} /\left(2 s f^{\prime \prime}+f^{\prime}\right)$, and obviously implies $g_{\mu \nu}^{*} k^{\mu} k^{\nu} \leq 0$ if conditions (b) and (c) are satisfied. The causal cone of the scalar field is thus timelike or null with respect to the Einstein metric $g_{\mu \nu}^{*}$. Such a condition (c) would be extremely constraining, since it would rule out notably any monomial $f(s)=s^{n}$, which would violate either (a) or (c) depending on the parity of $n$. However, let us underline that (c) is actually not required for the consistency of the model. Indeed, even if $\varphi$ propagates superluminally, this does not ruin the causality of the model. Thanks to condition (b), the causal cone of $\varphi$ never opens totally, i.e., always admit a class of Cauchy surfaces which can be defined in its exterior. In the general situation where different fields have different causal cones, it suffices that their union still admits a nonvanishing exterior where one may consistently define initial data for all of them simultaneously; see Fig. 1. This becomes quite clear by shifting our viewpoint. Let us assume that the union of all causal cones may be embedded in the interior of a wider cone which never totally opens, and let us define space and time with respect to this exterior cone. Since all fields propagate within this cone, there is no more causal problem than in special relativity where particle worldlines lie within the standard lightcone. Causal pathologies are still possible, like in GR itself which admits solutions containing closed timelike curves (CTC), like the Gödel universe or the interior of the Kerr solution. Since the global topology of the Universe is not imposed by our local field equations, it is necessary to assume that it does not involve any CTC to ensure causality. In our example of different fields having different causal cones, one thus just needs to assume that spacetime does not admit any CTC with respect to the wider 


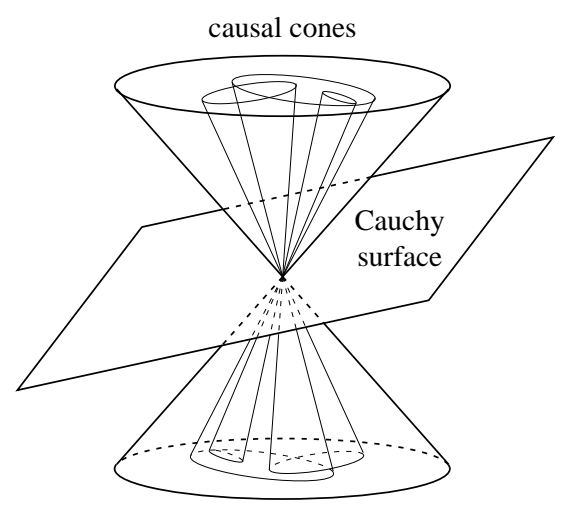

FIG. 1: In a theory where different fields have different causal cones, it suffices that their union be embedded in a wider cone for local causality to be satisfied. Initial data for all the fields simultaneously may be specified on a surface exterior to the wider cone, i.e., spacelike with respect to each cone. If the topology of spacetime is such that there does not exist any CTC with respect to the wider cone, then causality is preserved, although some fields may propagate faster than light (i.e., faster than electromagnetic waves, a mere particular case of matter field).

cone surrounding all of them. Causal paradoxes due to superluminal propagation, like the nice one exhibited in Sec. III. 2 of [102], are thus more a matter of global assumptions than a problem with local field equations. ${ }^{5}$ We refer to Ref. [103] for a more detailed discussion of this subtle issue (see also Secs. IIIC below). The very recent article [104] claims that microscopically Lorentz-invariant particles cannot give rise to superluminal signals, but this conclusion does not take into account their possible self-interactions via their kinetic term, like $f(s)$ in our present case of k-essence models (2.13). As shown above, superluminal signals do occur when $f^{\prime \prime}(s)>0$, although the theory is microscopically Lorentz-invariant - and causal when the hyperbolicity conditions (a) and (b) are satisfied. The causal cone of the scalar field $\varphi$ is either interior or exterior to the light cone defined by $g_{\mu \nu}^{*}$ (depending on the local value of $s$ ), but it always remains a cone thanks to these conditions. At each spacetime point, any surface exterior to the wider of these two cones may be used as a Cauchy surface to impose initial data. Of course, a surface lying between the two cones behaves as a spacelike one for the thinner cone but as a timelike one for the thicker. Some causal paradoxes discussed in the literature (including in [101]) are actually based on the improper use of such intermediate surfaces. They are clearly not consistent Cauchy surfaces for the fastest field (i.e., the wider cone), so that data are necessarily constrained on them. The conclusion of such paradoxes, namely that initial data are constrained, is thus actually hidden in the use of these intermediate surfaces.

\footnotetext{
${ }^{5}$ In Ref. [102], a CTC is exhibited by considering a background involving two bubbles of a scalar field with a fast relative motion. If one assumes that all clocks can be globally synchronized by using only electromagnetic waves, then such a CTC does exist. However, although this hypothesis sounds extremely natural, there also exist spacetimes in which there are no CTCs for any field, and in such a case, light within a given bubble of scalar field does not define the same synchronization as within the other bubble. The CTC exhibited in [102] becomes then an artifact of gluing independent coordinate systems at the edges of the bubbles.
} 


\section{F. MOND as a RAQUAL model}

As mentioned above, Ref. [27] proved that RAQUAL models (2.13) may reproduce the MOND gravitational potential. Indeed, if $f(s)$ is assumed not to depend explicitly on $\varphi$, if the potential $V(\varphi)$ vanishes, and if one chooses an exponential (Brans-Dicke-like) matterscalar coupling function $A(\varphi)=\exp (\alpha \varphi)$, Eq. (2.14) reduces to

$$
\nabla_{\mu}^{*}\left[f^{\prime}(s) \nabla_{*}^{\mu} \varphi\right]=-\frac{4 \pi G}{c^{4}} \alpha T^{*},
$$

where $T^{*} \equiv g_{\mu \nu}^{*} T_{*}^{\mu \nu}$ denotes the trace of the matter energy-momentum tensor $T_{*}^{\mu \nu} \equiv$ $\left(2 c / \sqrt{-g_{*}}\right) \delta S_{\text {matter }} / \delta g_{\mu \nu}^{*}$. In order to recover the right Newtonian limit for large accelerations $a>a_{0}$, one may follow Ref. [27] and impose ${ }^{6} f^{\prime}(s) \rightarrow 1$ for large positive values of $s$. In such a case, the scalar field reads $\varphi \approx-\alpha G M / r c^{2}$ near a body of mass $M$, and test particles feel an extra potential $\alpha \varphi c^{2} \approx-\alpha^{2} G M / r$ adding up to the standard gravitational potential $-G M / r$ mediated by the spin-2 interaction (i.e., by the Einstein metric $g_{\mu \nu}^{*}$ ). The total potential $-G\left(1+\alpha^{2}\right) M / r$ is thus of the Newtonian type, with a renormalized effective gravitational constant $G_{\text {eff }}=G\left(1+\alpha^{2}\right)$ (see for instance [89] and line (a) of Fig. [5] in Sec. III below). Precision tests in the solar system are however sensitive to post-Newtonian corrections, and they prove that the scalar contribution must be negligible. Indeed, the parametrized post-Newtonian (PPN) parameter $\gamma^{\mathrm{PPN}}$ assumes the value $\gamma^{\mathrm{PPN}}=1-2 \alpha^{2} /\left(1+\alpha^{2}\right)$ in the present (conformally-coupled) scalar-tensor framework [see Refs. 11, 89] and Eq. (33.4) below], and the impressive experimental bound $\left|\gamma^{\mathrm{PPN}}-1\right|<2 \times 10^{-5}$ obtained in [88] from the observation of the Cassini spacecraft implies therefore

$$
\alpha^{2}<10^{-5}
$$

Instead of imposing $f^{\prime}(s) \rightarrow 1$ for large values of $s$, one may thus also recover the right Newtonian limit by choosing a decreasing function $f^{\prime}(s)$, such that the scalar contribution is even smaller. Condition (b) should however still be satisfied, thereby constraining the slope of $f^{\prime}(s)$.

But the crucial feature of Eq. (2.15) is that it also allows us to reproduce the MOND potential for small accelerations $a<a_{0}$. Indeed, if $f^{\prime}(s) \approx \ell_{0} \sqrt{s}$ for small and positive values of $s$, where $\ell_{0}$ is a constant having the dimension of a length, one gets $\left(\partial_{r} \varphi\right)^{2} \approx$ $\alpha G M / \ell_{0} c^{2} r^{2}$ near a spherical body of mass $M$. Test particles therefore feel, in addition to the usual Newtonian potential $-G M / r$, an extra potential $\alpha \varphi c^{2} \approx \sqrt{\alpha^{3} G M c^{2} / \ell_{0}} \ln r$ which reduces to the MOND one $\sqrt{G M a_{0}} \ln r$ for $\ell_{0}=\alpha^{3} c^{2} / a_{0}$. A simple way to connect this MOND limit to the above Newtonian one is to choose for instance $f^{\prime}(s)=\sqrt{\bar{s}} / \sqrt{1+\bar{s}}$, where $\bar{s} \equiv \alpha^{6} c^{4} s / a_{0}^{2}$ is dimensionless. The shape of this function is illustrated in Fig. 2, By integrating this expression, one can thus conclude that the precise function $f(s)=$ $\left(a_{0}^{2} / \alpha^{6} c^{4}\right)\left[\sqrt{\bar{s}(1+\bar{s})}-\sinh ^{-1}(\sqrt{\bar{s}})\right]$, or any other one having the same asymptotic behaviors for large and small positive values of $s$, allows us to reproduce the MOND dynamics.

However, the authors of Ref. [27] noticed that the scalar field propagates superluminally in the MOND (small positive $s$ ) regime, since $f^{\prime \prime}(s) \approx \frac{1}{2} \ell_{0} / \sqrt{s}>0$ contradicts condition

\footnotetext{
${ }^{6}$ Note that imposing $f^{\prime}(s) \rightarrow$ const. is strictly equivalent, since the arbitrary constant can be absorbed in a redefinition of the matter-scalar coupling constant $\alpha$. In the following, the experimental constraints on $\alpha$ that we quote correspond to the choice $f^{\prime}(s) \rightarrow 1$ in the Newtonian regime.
} 


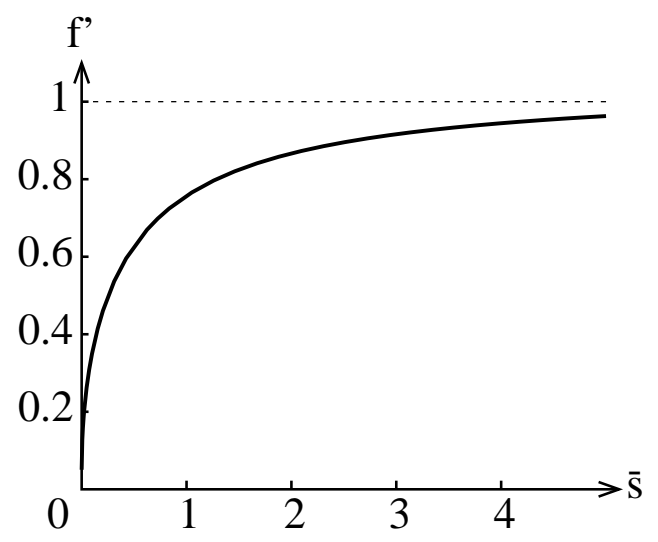

FIG. 2: A simple function $f^{\prime}(s)$ reproducing the MOND dynamics for small $s$ (i.e., large distances) and the Newtonian one for large $s$ (i.e., small distances).

(c) above. This is the reason why they discarded this model, although we underlined above that such a superluminal propagation actually does not threaten causality, provided the hyperbolicity conditions (a) and (b) are satisfied. In fact, the only experimental constraints we have on the existence of several causal cones is in favor of superluminal fields! As we will discuss in Sec. IVC, if light (and thus matter) travelled faster than some field to which it couples, then it would emit Cerenkov radiation of this field. High-energy cosmic rays would thus be significantly suppressed.

It thus seems that the above RAQUAL model reproducing the MOND dynamics is $a$ priori consistent. However, it presents several other difficulties. The main one has been immediately recognized and addressed in the literature: Because of the specific form of the matter-scalar coupling assumed in action (2.13), this model does not reproduce the observed light deflection by "dark matter". We will devote Sec. III below to this crucial problem. Let us here mention the other problems that we noticed, and which do not seem to have been discussed in the literature.

First of all, the above function $f(s)$ is clearly not defined for negative values of $s$. One may try to replace $s$ by its absolute value $|s|$, and also multiply globally $f(s)$ by the sign of $s$ in order to still satisfy conditions (a) and (b). However, this would not cure the serious problem occurring at $s=0$. Indeed, since $f(s) \rightarrow\left(2 \alpha^{3} c^{2} / 3 a_{0}\right) s \sqrt{|s|}$ for small values of $s$, the strict inequality (b) is not satisfied at $s=0$. In other words, the scalar field equation (2.14) or (2.15) is no longer hyperbolic on the hypersurfaces where $s$ vanishes, and the scalar degree of freedom cannot cross them. Since $s \approx\left(\partial_{i} \varphi\right)^{2} \geq 0$ when considering the local physics of clustered matter, but $s \approx-\dot{\varphi}^{2} \leq 0$ when considering the cosmological evolution of the Universe, there always exist such singular surfaces around clusters. Therefore, this model cannot describe consistently both cosmology and galaxies, unless independent solutions are glued by hand on the singular hypersurfaces $s=0$. However, a simple cure to this discontinuity would be to consider, for small values of $s$, a function

$$
f^{\prime}(s)=\varepsilon+\sqrt{|\bar{s}|}
$$

where $\varepsilon$ is a small dimensionless positive number. Then both conditions (a) and (b) would obviously be satisfied even at $s=0$. In other words, the RAQUAL model (2.13) with 


$$
\begin{aligned}
& V(\varphi)=0, A(\varphi)=\exp (\alpha \varphi) \text { and } \\
& \qquad f(s)=\varepsilon s+\frac{a_{0}^{2} \operatorname{sgn}(s)}{\alpha^{6} c^{4}}\left[\sqrt{|\bar{s}|(1+|\bar{s}|)}-\sinh ^{-1}(\sqrt{|\bar{s}|})\right],
\end{aligned}
$$

where $\bar{s} \equiv \alpha^{6} c^{4} s / a_{0}^{2}$, does reproduce the MOND dynamics and is free of mathematical inconsistencies. Of course, there is no reason why this specific function, for negative values of $s$, should reproduce the right cosmological behavior. One may obviously look for other functions of $s<0$ connecting smoothly to (2.18) for small values of $|s|$, provided conditions (a) and (b) above remain always satisfied. Therefore, Eq. (2.18) should just be considered as an example of a mathematically consistent RAQUAL model.

A second difficulty is that the above model is rather fine tuned, since it needs the introduction of a small dimensionless constant $\varepsilon$ besides Milgrom's MOND acceleration constant $a_{0} \approx 1.2 \times 10^{-10} \mathrm{~m} \cdot \mathrm{s}^{-2}$. Indeed, the presence of $\varepsilon$ in Eqs. (2.17), (2.18) notably implies that Newtonian gravity is recovered at very large distances, the MOND regime manifesting only at intermediate ranges [103]. When $s \rightarrow 0$, the derivative $f^{\prime}(s)$ entering Eq. (2.15) tends to $\varepsilon$, so that the scalar field reads $\varphi \approx-(\alpha / \varepsilon) G M / r c^{2}$ faraway from a body of mass $M$. In this regime, the total gravitational potential felt by a test particle reads $-G\left(1+\alpha^{2} / \varepsilon\right) M / r$, and is therefore of the Newtonian form with a renormalized gravitational constant $G_{\infty}=G\left(1+\alpha^{2} / \varepsilon\right)$, where the subscript refers to the fact that the above form of the potential holds exactly when $r \rightarrow \infty$ (remind that, if $r \rightarrow 0$, the effective gravitational constant is given by $G_{\text {eff }}=G\left(1+\alpha^{2}\right)$ ). Let us compute the range of distances $r$ for which the MOND force dominates the Newtonian contributions. One needs of course $f^{\prime}(s) \propto \sqrt{s}$ and thus $\varepsilon \ll \sqrt{\bar{s}}$. On the other hand, the MOND force dominates the Newtonian one if $r \gg \sqrt{G M / a_{0}}$. Using $s \approx\left(\partial_{r} \varphi\right)^{2} \approx G M a_{0} / \alpha^{2} c^{4} r^{2}$, we thus find that the MOND force dominates within the following range of distances $\sqrt{G M / a_{0}} \ll r \ll\left(\alpha^{2} / \varepsilon\right) \sqrt{G M / a_{0}}$. Since solar system tests impose $\alpha^{2}<10^{-5}$, Eq. (2.16) , and since rotation curves of galaxies may be flat up to $r \sim 10 \sqrt{G M / a_{0}}[105]$, one needs therefore $\varepsilon \ll 10^{-6}$. This illustrates the fine tuning required to define a consistent RAQUAL model even for $s \rightarrow 0$.

On the other hand, Eq. (2.17) has two great advantages, besides the fact that it cures the singularity at $s=0$. First, the fact that the field assumes a Newtonian form at large distances, $r>\left(\alpha^{2} / \varepsilon\right) \sqrt{G M / a_{0}}$, ensures that a body of mass $M$ does not create a static (scalar) field with infinite energy, contrary to the case where $f^{\prime}(s) \sim \sqrt{s}$ for small $s$ [27]. Secondly, the presence of $\varepsilon$ naturally leads to a theory where the local constant of gravity $G_{\text {eff }}=G\left(1+\alpha^{2}\right)$ may differ from $G_{\infty}$, i.e., the one in regions of spacetime where the scalar field gradient is small. This may have some interesting applications in cosmology. Indeed, provided that $\sqrt{|\bar{s}|} \lesssim \varepsilon$, i.e., when $\dot{\varphi}^{2} \lesssim \varepsilon a_{0}^{2} / \alpha^{6} c^{2}$ in a cosmological context, a homogeneous Universe is described by Friedman equations ${ }^{7}$ but the baryonic matter contributes as $G_{\infty} \rho_{\text {baryons. }}$ The effective density of matter reads therefore $\rho_{\text {baryons }} G_{\infty} / G_{\text {eff }}=\rho_{\text {baryons }}\left(1+\alpha^{2} / \varepsilon\right) /\left(1+\alpha^{2}\right) \approx \rho_{\text {baryons }}\left(1+\alpha^{2} / \varepsilon\right)$. In other words, baryonic matter may contribute effectively as if there were true (exotic) dark matter in that regime, provided that we tune the free parameter $\varepsilon$ so that $\alpha^{2} / \varepsilon \approx 10$, which is consistent with galactic dynamics. Since the cosmology of such models goes beyond the scope of the present paper, we will not investigate further this phenomenology. Let us however stress that there is here a potential threat for MOND-like theories. Indeed, bounds may be found

\footnotetext{
${ }^{7}$ Beware that the density and the pressure of the scalar field must also be taken into account.
} 
on the ratio $G_{\infty} / G_{\text {eff }}$ from the analysis of primordial nucleosynthesis, since the speed of the expansion of the Universe, and therefore the relative proportion of hydrogen and helium in the present Universe, depends on this ratio. Within the framework of the so-called Einsteinaether theories [106, 107], it was shown in Ref.[108] that one must have $\left|G_{\infty} / G_{\text {eff }}-1\right| \lesssim 1 / 8$ to be consistent with the data. Of course, in the above RAQUAL model of MOND, such a constraint only exists if the time derivative of the scalar field is small at the epoch of nucleosynthesis. In that case, the above bound would read $\alpha^{2} / \varepsilon<1 / 8$, and the MOND regime would therefore not exist at all (there is no more room for the flattening of rotation curves, as shown in the above paragraph). A solution would of course to impose a very small value of $\varepsilon$ such that $\dot{\varphi}^{2} \gg \varepsilon a_{0}^{2} / \alpha^{6} c^{2}$ at the nucleosynthesis epoch. However one should study the cosmology of such models to investigate in more detail this effect and make quantitative estimates; we leave this point for future work. ${ }^{8}$

Finally, the third (and the most serious) problem of such RAQUAL models of MOND, besides the one of light deflection discussed in Sec. III below, is that the MOND force $\propto \sqrt{G M a_{0}} / r$ starts manifesting at quite small distances. Indeed, the MOND potential $\propto \sqrt{G M a_{0}} \ln r$ appears at values of field gradient $\bar{s}$ such that $f^{\prime}(\bar{s}) \sim \sqrt{\bar{s}}$. This typically happens at $\bar{s} \lesssim 1$ for natural functions $f^{\prime}$ interpolating between the MOND and the Newtonian regimes, see Fig. 2. Using $\bar{s} \lesssim 1$ we find that the MOND potential appears at distance $r \gtrsim r_{\text {trans }}$ from a body of mass $M$, where

$$
r_{\text {trans }}=\alpha^{2} \sqrt{\frac{G M}{a_{0}}} .
$$

Since, in the present (conformally-coupled) scalar-tensor framework, the matter-scalar coupling constant $\alpha$ is severely constrained by solar-system tests, Eq. (2.16), we conclude that the extra MOND potential starts manifesting at a radius $r_{\text {trans }}=\alpha^{2} \sqrt{G M_{\odot} / a_{0}}<0.1 \mathrm{AU}$ in the solar system, where $M_{\odot}$ denotes the mass of the Sun. In other words, all planets (including Mercury, at 0.4 AU from the Sun) should feel a total gravitational potential $-G M_{\odot} / r+\sqrt{G M_{\odot} a_{0}} \ln r$, the first term coming from the spin-2 field $g_{\mu \nu}^{*}$, and the second one from the scalar field which is already in its MOND regime. This extra potential $\sqrt{G M_{\odot} a_{0}} \ln r$ (or force in $1 / r$ ) leads to deviations from the Newtonian behavior and is tightly constrained by tests of Kepler's third law; see [109] and Sec. VC below. Although this MONDian anomalous force remains numerically small with respect to the Newtonian one within the solar system, it is large with respect to post-Newtonian corrections $\propto 1 / c^{2}$, and its existence is definitely ruled out experimentally. In conclusion, although the RAQUAL model of Ref. [27] or its refinement (2.18) do predict a Newtonian behavior at distances $r \rightarrow 0$ (i.e., $s \rightarrow \infty$ ), planets are far enough to be already in the MOND regime! Note that this surprizing problem comes from the experimental bound (2.16), telling us that the scalar field must be very weakly coupled to matter to reproduce GR within the solar system. If one did not take it into account, by setting $\alpha=1$ for instance, one would easily reproduce Newtonian gravity up to $\sqrt{G M_{\odot} / a_{0}} \approx 7000 \mathrm{AU}$, and the MOND dynamics beyond. A reasonable value $\varepsilon \sim 1 / 10$ would also suffice for the refined model (2.18). However, this theory would be inconsistent by 5 orders of magnitude with post-Newtonian tests in the solar system, because the scalar field would be much too strongly coupled to matter.

\footnotetext{
${ }^{8}$ Note that this phenomenon is very likely to occur also in extended models we consider below (TeVeS-like theories), since they notably involve a k-essence scalar field whose kinetic term also needs to be cured at $s=0 ;$ see Sec. IIC
} 

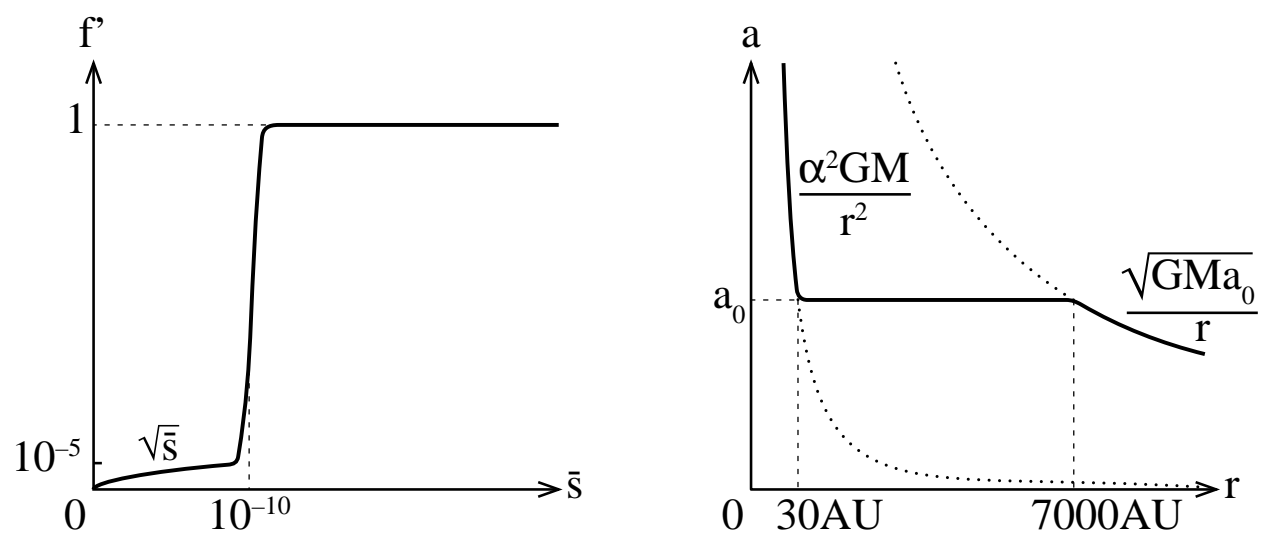

FIG. 3: Fine-tuned function $f^{\prime}(s)$ such that Newtonian and post-Newtonian predictions are not spoiled in the solar system, although the MOND dynamics is predicted at large distances. The right panel displays the quite unnatural contribution of the scalar field to the acceleration of a test mass, as a function of its distance with respect to the Sun.

A possible solution to the above problem would be to fine tune the function $f(s)$ even further. In order to get the MOND regime for $r \gtrsim \sqrt{G M / a_{0}}$, as required by galaxy rotation curves, one would need $f^{\prime}(s) \approx \sqrt{\bar{s}}$ for $\bar{s} \lesssim \alpha^{4}<10^{-10}$. On the other hand, in order to obtain the Newtonian regime within the solar system, say for $r \lesssim r_{\max } \sim 20$ or 30 AU, one would need $f^{\prime}(s) \approx 1$ for $\bar{s} \gtrsim\left(\alpha^{4} G M_{\odot} / a_{0} r_{\max }^{2}\right)^{2} \sim 10^{-10}$ (this second occurrence of $10^{-10}$ is a numerical coincidence). Therefore, there would exist a brutal transition between the MOND and Newtonian regimes around $\bar{s} \sim 10^{-10}$. Not only the introduction of such a small dimensionless number would be quite unnatural, but this model would also predict that the anomalous acceleration caused by the scalar field remains approximately equal to the constant ${ }^{9} a_{0}$ between 30 and $7000 \mathrm{UA}$. As illustrated in Fig. 3, this would be a way to reconcile the MOND acceleration $\sqrt{G M a_{0}} / r$ at large distances with the experimentally small Newtonian contribution $\alpha^{2} G M / r^{2}$ of the scalar field at small distances. Although this is not yet excluded experimentally, it would however suffice to improve by one order of magnitude the post-Newtonian constraint on $\alpha^{2}$ to rule out such a fine-tuned model (the planned astrometric experiment GAIA [110] should reach the $10^{-6}$ level for $\alpha^{2}$, and the proposed LATOR mission [111, 112] should even reach the $10^{-8}$ level). Therefore, one should not consider it seriously.

An a priori better solution to the above problem would be to recall that the Newtonian limit $f^{\prime}(s) \approx 1$ is actually unnecessary. Since the metric $g_{\mu \nu}^{*}$ already generates a Newtonian potential $-G M / r$, it suffices that the scalar field contribution remain negligible (even at the post-Newtonian level) in the solar system. One may try a function $f^{\prime}(s)$ whose shape looks like the one displayed in Fig. 4, for instance

$$
f^{\prime}(s)=\varepsilon+\frac{\sqrt{|\bar{s}|}}{(1+|\bar{s}|)^{1+1 / n}},
$$

\footnotetext{
${ }^{9}$ Note that the MOND constant $a_{0}$ is too small by a factor 7 to account for the anomalous acceleration of the two Pioneer spacecrafts. Actually, we will also see in Sec. $\mathrm{VC}$ below that there exists a crucial difference between the MOND dynamics and the Pioneer anomaly.
} 


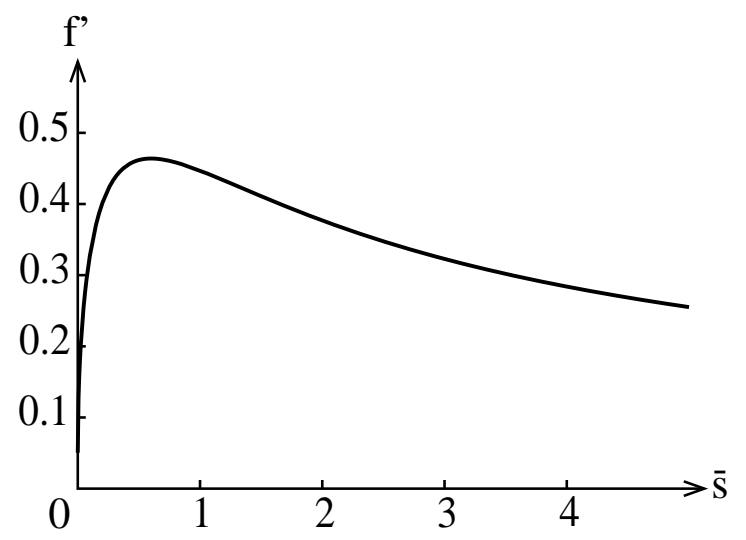

FIG. 4: Typical shape of a function $f^{\prime}(s)$ such that scalar-field deviations from GR are negligible at small distances.

where $n$ is a positive constant and as before $\bar{s} \equiv \alpha^{6} c^{4} s / a_{0}^{2}$. Then the transition occurs again at $\bar{s} \sim 1$, i.e., around $r_{\text {trans }}=\alpha^{2} \sqrt{G M / a_{0}}$, but the difference with the previous model is that $\alpha$ is no longer constrained by post-Newtonian tests. Indeed, in the large- $s$ limit, Eq. (2.18) shows that the force mediated by the scalar field reads $\alpha \partial_{r} \varphi c^{2}=\left(a_{0} / \alpha^{2}\right)\left(r / r_{\text {trans }}\right)^{n}$. Therefore, even if one imposes a strong scalar-matter coupling constant $\alpha=1$, so that the NewtonMOND transition occur at $r_{\text {trans }}=\sqrt{G M / a_{0}}$ as expected, the anomalous acceleration caused by the scalar field will be of order $10^{-4 n} a_{0}$ in the solar system, at $r \sim 1$ AU. A value $n \gtrsim 2$ suffices thus for this anomalous force to be negligible even with respect to post-Newtonian relativistic effects. However, it is easy to check that the function (2.20) does not satisfy condition (b) for large values of $s$, and therefore that the scalar field equation is no longer hyperbolic for small enough distances. This suffices to rule out this class of models.

In conclusion, the above discussion illustrates that RAQUAL models are severely constrained, although they involve a free function $f(s)$ defining the kinetic term of the scalar field. Contrary to some fears in the literature, the possible superluminal propagations do not threaten causality, and the two conditions (a) and (b) are the only ones which must be imposed to guarantee the field theory's consistency. For instance, monomials $f(s)=s^{n}$ are allowed if $n$ is positive and odd [except on the possible hypersurfaces where $s$ vanishes, which would violate the strict inequality (b)]. However, when taking into account simultaneously these two consistency conditions and experimental constraints, it seems difficult to reproduce the MOND dynamics at large distances without spoiling the Newtonian and postNewtonian limits in the solar system. It seems necessary to consider unnatural functions $f(s)$ involving small dimensionless numbers.

In the next section, we will address the problem of light deflection, and recall the solution which has been devised in the literature. This solution will at the same time release the experimental constraint on the matter-scalar coupling constant $\alpha$ obtained from accurate measurement of the post-Newtonian parameter $\gamma^{\mathrm{PPN}}$ within the solar system, and therefore cure the above fine-tuning problem. On the other hand, we will show in Sec. IVD that the analysis of binary-pulsar data also imposes a bound on $\alpha$, so that the problem of the fine-tuning of the free function $f$ will anyway remain. 
(a)

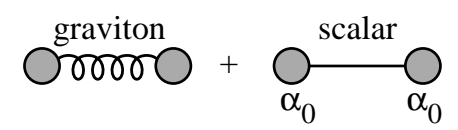

(b)

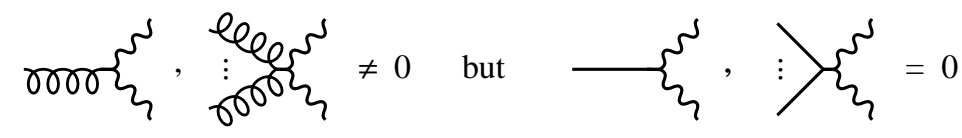

(c)

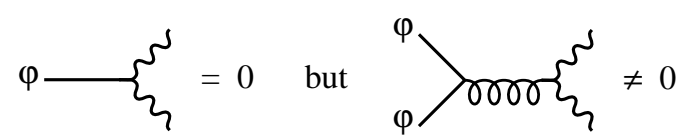

FIG. 5: Feynman diagrams in scalar-tensor theories, where straight, curly and wavy lines represent respectively the scalar field, gravitons and photons. Matter sources are represented by blobs. (a) Diagrammatic interpretation of the effective gravitational constant $G_{\text {eff }}=G\left(1+\alpha_{0}^{2}\right)$, where each vertex connecting matter to one scalar line involves a factor $\alpha_{0}$. (b) Photons are directly coupled to gravitons but not to the scalar field. (c) Photons feel nevertheless the scalar field indirectly, via its influence on gravitons: The energy-momentum tensor of the scalar field generates a curvature of the Einstein metric $g_{\mu \nu}^{*}$ in which electromagnetic waves propagate.

\section{THE PROBLEM OF LIGHT DEFLECTION}

\section{A. Conformally-coupled scalar field}

Both scalar-tensor theories (2.12) and RAQUAL models (2.13) assume a conformal relation $\tilde{g}_{\mu \nu}=A^{2}(\varphi) g_{\mu \nu}^{*}$ between the physical and Einstein metrics. The inverse metrics are thus related by $\tilde{g}^{\mu \nu}=A^{-2}(\varphi) g_{*}^{\mu \nu}$, and their determinants read $\tilde{g}=A^{2 d}(\varphi) g_{*}$ in $d$ spacetime dimensions. Since all matter fields, including gauge bosons, are assumed to be minimally coupled to $\tilde{g}_{\mu \nu}$, this leads to a simple prediction for the behavior of light in such models. Indeed, the action of electromagnetism is conformal invariant in $d=4$ dimensions:

$$
\begin{aligned}
S_{\mathrm{EM}} & =\int \frac{d^{4} x}{c} \frac{\sqrt{-\tilde{g}}}{4} \tilde{g}^{\mu \rho} \tilde{g}^{\nu \sigma} F_{\mu \nu} F_{\rho \sigma} \\
& =\int \frac{d^{4} x}{c} \frac{A^{4}(\varphi) \sqrt{-g_{*}}}{4}\left[A^{-2}(\varphi) g_{*}^{\mu \rho}\right]\left[A^{-2}(\varphi) g_{*}^{\nu \sigma}\right] F_{\mu \nu} F_{\rho \sigma} \\
& =\int \frac{d^{4} x}{c} \frac{\sqrt{-g_{*}}}{4} g_{*}^{\mu \rho} g_{*}^{\nu \sigma} F_{\mu \nu} F_{\rho \sigma} .
\end{aligned}
$$

Therefore, light is only coupled to the spin-2 field $g_{\mu \nu}^{*}$, but does not feel at all the presence of the scalar field $\varphi$. In terms of Feynman diagrams, there exist nonvanishing vertices connecting one or several gravitons to two photon lines, but the similar vertices connecting photons to scalar lines all vanish; this is illustrated in line (b) of Fig. 5, It is then obvious that light behaves strictly as in GR, in a geometry described by the Einstein metric $g_{\mu \nu}^{*}$. In particular, the light deflection angle caused by a spherical body of mass $M$ must be given by the same expression as in GR (at lowest order)

$$
\Delta \theta=\frac{4 G M}{b c^{2}}
$$

where $b$ denotes the impact parameter of the light ray. An even simpler way to prove that light propagates in the Einstein metric $g_{\mu \nu}^{*}$, without feeling the scalar field, is to note that 
its geodesic equation reads $\tilde{g}_{\mu \nu} d x^{\mu} d x^{\nu}=0$ in the eikonal approximation. Dividing by the nonvanishing factor $A^{2}(\varphi)$, this equation implies $g_{\mu \nu}^{*} d x^{\mu} d x^{\nu}=0$, giving thus the standard geodesic equation for null rays in the metric $g_{\mu \nu}^{*}$. [It is interesting to note that this second reasoning would remain valid even if the action of electromagnetism were not conformal invariant, for instance in dimension $d \neq 4$, or even if one multiplied it by an explicit scalarphoton coupling function $B^{2}(\varphi)$, thereby violating the weak equivalence principle. Then it is straightforward to prove that the nonvanishing scalar-photon vertices would only affect the amplitude of electromagnetic waves, in the eikonal approximation, but not their polarization nor their trajectory. One of us (G.E.F.) discussed this result with B. Bertotti several years ago, but it has been recently rediscovered in [113].]

However, the crucial difference with GR is that massive matter does feel the scalar field, via the matter-scalar coupling function $A(\varphi)$. One may expand this function around the background value $\varphi_{0}$ of the scalar field as

$$
\ln A(\varphi)=\text { const. }+\alpha_{0}\left(\varphi-\varphi_{0}\right)+\frac{1}{2} \beta_{0}\left(\varphi-\varphi_{0}\right)^{2}+\cdots,
$$

where $\alpha_{0}, \beta_{0}, \ldots$, are dimensionless constants. In usual scalar-tensor theories (2.12), as well as in the Newtonian regime of RAQUAL models (2.13), the lowest-order contribution of the scalar field to the gravitational potential felt by a test mass reads then $-\alpha_{0}^{2} G M / r$ [1, 89]. This generalizes the results recalled in Sec. IIF above in the particular case of an exponential coupling function $A(\varphi)=e^{\alpha \varphi}$. This spin-0 contribution adds up to the standard Newtonian potential $-G M / r$ caused by the spin-2 interaction (i.e., via the Einstein metric $\left.g_{\mu \nu}^{*}\right)$, so that the total gravitational potential remains of the Newtonian form but with an effective gravitational constant $G_{\text {eff }}=G\left(1+\alpha_{0}^{2}\right)$; see line (a) of Fig. 5. In other words, when one determines the mass of the Sun by analyzing the orbits of the planets, one actually measures $G_{\text {eff }} M$ instead of the bare $G M$. Therefore, if one expresses the light deflection angle (3.2) in terms of the observable quantity $G_{\text {eff }} M$, one gets

$$
\Delta \theta=\frac{4 G_{\mathrm{eff}} M}{\left(1+\alpha_{0}^{2}\right) b c^{2}} \leq \frac{4 G_{\mathrm{eff}} M}{b c^{2}} .
$$

For a given value of the observed constant $G_{\text {eff }} M$, general relativity would have predicted $4 G_{\text {eff }} M / b c^{2}$. In conclusion, the light deflection angle is actually smaller in scalar-tensor theories than in GR, although photons are strictly decoupled from the scalar field! This paradoxical result is simply due to the fact that we need to compare the measured angle $\Delta \theta$ with another observable, $G_{\text {eff }} M$, which is influenced by the scalar field.

This reduction of light deflection in scalar-tensor theories has been immediately recognized as a serious problem for MOND-like field theories [28, 29]. Indeed, if one interprets data within GR, the presence of dark matter suggested by galaxy rotation curves is confirmed by weak lensing observations [114, 115, 116]. Therefore, in a modified-gravity theory avoiding the assumption of dark matter, one should predict simultaneously a larger gravitational potential and a larger light deflection. However, the interpretation of data is slightly more subtle than in the solar system, because there exist at least three different notions of mass which may be defined for a galaxy: (i) its baryonic mass $M_{\mathrm{b}}$, assumed to be proportional to its luminous mass $M_{\mathrm{L}}$; (ii) its total mass (baryonic plus "dark") evaluated from its rotation curve, that we will denote $M_{\text {tot }}^{\text {rot }}$ and (iii) its total mass evaluated from weak lensing, that we will denote $M_{\text {tot }}^{\text {lens }}$. The only conclusion driven from the above result (3.4) is that the deflecting mass is smaller than the gravitational one in scalar-tensor theories:

$$
M_{\mathrm{tot}}^{\mathrm{lens}} \leq M_{\mathrm{tot}}^{\mathrm{rot}}
$$


Note that this conclusion is also valid in the class of RAQUAL models reproducing the MOND dynamics that we discussed in Sec. IIF above. Indeed, the MOND force was caused by the exchange of a scalar particle [cf. line (a) of Fig. 15], i.e., by the conformal factor $A^{2}(\varphi)$ relating the physical metric $\tilde{g}_{\mu \nu}$ to the Einstein one $g_{\mu \nu}^{*}$. Since light only feels the latter, the total mass evaluated from weak lensing is obviously smaller that the one evaluated from rotation curves.

On the other hand, it should be underlined that Eq. (3.4) does not imply $M_{\text {tot }}^{\text {lens }} \leq M_{\mathrm{b}}$, contrary to some erroneous or imprecise claims in the literature (see, e.g., [29]). Indeed, the stress-energy tensor of the scalar field does contribute to the curvature of the Einstein metric $g_{\mu \nu}^{*}$, so that light deflection may be larger than if only baryonic matter was present. This is illustrated in terms of Feynman diagrams in line (c) of Fig. 55: Although the scalarphoton vertex vanishes, light may nevertheless feel the scalar field indirectly via a graviton exchange. The reason why this does not appear in Eqs. (3.2) or (3.4) above is because we derived them at lowest order in powers of $1 / c^{2}$, and in usual scalar-tensor theories or in the Newtonian regime of RAQUAL models. But when considering their MOND regime, one cannot any longer perform a naive post-Newtonian expansion. Indeed, the stress-energy of the scalar field may become non-negligible with respect to that of baryonic matter, and even dominate it, although this scalar field has been generated by its coupling to matter. This mere sentence gives us a hint that such a model may be unstable, but one should note that $M_{\mathrm{tot}}^{\text {lens }} \gg M_{\mathrm{b}}$ is indeed possible in RAQUAL models.

We will provide an explicit example in Sec. IIIB below, although it does not reproduce the right MOND dynamics. Its aim is just to underline that the derivation of the inequality $M_{\text {tot }}^{\text {lens }} \leq M_{\mathrm{b}}$ proposed in Ref. [29] is incorrect. It is instructive to locate where its reasoning fails. It writes the deflection angle in terms of the $T_{r}^{r}$ component of the total stress-energy tensor, and decomposes it in two contributions, due to matter and to the scalar field. It happens that the latter is negative. However, what was identified as the matter contribution to $T_{r}^{r}$ happens not to vanish outside matter. Therefore, the decomposition was flawed, and part of the positive contribution of the scalar field to light deflection was actually attributed to matter. The simplest way to understand that a scalar field may indeed increase light deflection is to consider it as a dark matter fluid, without any coupling to baryonic matter and assuming GR as the correct theory of gravity. If the scalar field is massive enough, it will obviously cluster and contribute to the total gravitational potential deflecting light.

Actually, a violation of inequality (3.5) is a priori also possible when the scalar's stressenergy contributes significantly to the curvature of the Einstein metric $g_{\mu \nu}^{*}$. Indeed, specific models could predict a negligible contribution to the time component $g_{00}^{*}$ but a significant one to the spatial components $g_{i j}^{*}$. In such a case, test particles would feel the standard Newtonian potential $-\frac{1}{2}\left(1+g_{00}^{*}\right) c^{2} \approx-G M / r$ [and the extra scalar force caused by the conformal factor $A^{2}(\varphi)$, which may be tuned to be negligible], but light bending would be directed affected by the scalar-induced corrections entering $g_{i j}^{*}$. Therefore, $M_{\mathrm{tot}}^{\text {lens }}>M_{\mathrm{tot}}^{\mathrm{rot}}$ seems possible. However, such models may be difficult to construct if one imposes the positivity of energy, as in Ref. [29]. We will not attempt to study them here, since there is anyway no experimental evidence for $M_{\text {tot }}^{\text {lens }}>M_{\text {tot }}^{\text {rot }}$. Weak-lensing observations only impose $M_{\mathrm{tot}}^{\text {lens }} \sim M_{\mathrm{tot}}^{\mathrm{rot}}[114,115,116$.

Let us end this section by recalling that the conclusion of Ref. [29] remains correct in a limiting case which is often implicitly assumed in MOND-like field theories [42]: If the stress-energy of the scalar field is negligible with respect to that of baryonic matter, then the curvature of the Einstein metric $g_{\mu \nu}^{*}$ is basically generated by $M_{\mathrm{b}}$ alone. Since light only 
feels $g_{\mu \nu}^{*}$, weak lensing provides a measure of $M_{\mathrm{tot}}^{\text {lens }} \approx M_{\mathrm{b}}$. In other words, light is only deflected by baryonic matter, whereas experiment tells us that the much greater amount of "dark matter" does deflect it too. We will review in Secs. IIIC and IIID how the same authors as Ref. [29] devised a clever way to solve this crucial difficulty of RAQUAL models.

\section{B. A RAQUAL example increasing light deflection}

Let us illustrate that RAQUAL models may predict a much larger light deflection than if there were baryonic matter alone in GR, i.e., that $M_{\mathrm{tot}}^{\text {lens }} \gg M_{\mathrm{b}}$ is possible. We consider the action

$$
S=\frac{c^{4}}{16 \pi G} \int \frac{d^{4} x}{c} \sqrt{-g_{*}}\left\{R^{*}-\ell_{0}^{2(n-1)} s^{n}\right\}+S_{\text {matter }}\left[\psi ; \tilde{g}_{\mu \nu}=A^{2}(\varphi) g_{\mu \nu}^{*}\right],
$$

where as before $s \equiv g_{*}^{\mu \nu} \partial_{\mu} \varphi \partial_{\nu} \varphi, \ell_{0}$ is a length scale (e.g. $\ell_{0} \propto c^{2} / a_{0}$ in MOND-like models), and $n$ is a positive odd integer. As underlined at the end of Sec. III, such a model satisfies the two consistency conditions (a) and (b), except on hypersurfaces where $s$ vanishes. It suffices to add a small standard kinetic term $-\varepsilon s$ (with $\varepsilon>0$ ) to the above action to cure the singularity on such hypersurfaces. However, we will not take into account this refinement because the above model will anyway not reproduce the correct MOND dynamics.

The field equations deriving from action (3.6) read

$$
\begin{aligned}
R_{\mu \nu}^{*}-\frac{1}{2} g_{\mu \nu}^{*} R^{*} & =\frac{8 \pi G}{c^{4}}\left(T_{\mu \nu}^{* \text { matter }}+T_{\mu \nu}^{* \varphi}\right) \\
n \ell_{0}^{2(n-1)} \nabla_{\mu}^{*}\left(s^{n-1} \partial_{*}^{\mu} \varphi\right) & =-\frac{8 \pi G}{c^{4}} \frac{d \ln A(\varphi)}{d \varphi} T_{\text {matter }}^{*}
\end{aligned}
$$

where the stress-energy tensor of the scalar field is given by

$$
\frac{8 \pi G}{c^{4}} T_{\mu \nu}^{* \varphi}=\left(\ell_{0}^{2} s\right)^{n-1}\left(n \partial_{\mu} \varphi \partial_{\nu} \varphi-\frac{1}{2} s g_{\mu \nu}^{*}\right) .
$$

Let us choose for instance an exponential (Brans-Dicke-like) matter-scalar coupling function $A(\varphi)=e^{\alpha \varphi}$, so that $d \ln A(\varphi) / d \varphi=\alpha$ is constant. The solution of Eq. (3.7b), around a spherical body of mass $M$, is then easy to write at lowest order (i.e., neglecting the curvature of spacetime):

$$
\varphi=\varphi_{0}+\frac{2 n-1}{2 n-3}\left(\frac{2 \alpha G M \ell_{0}}{n r^{2} c^{2}}\right)^{1 /(2 n-1)} \frac{r}{\ell_{0}} .
$$

The stress-energy tensor of the scalar field reads thus

$$
\frac{8 \pi G}{c^{4}} T_{\mu \nu}^{* \varphi}=\frac{\alpha G M}{\ell_{0} r^{2} c^{2}}\left(\frac{2 \alpha G M \ell_{0}}{n r^{2} c^{2}}\right)^{1 /(2 n-1)}\left(2 \delta_{\mu}^{r} \delta_{\nu}^{r}-\frac{1}{n} g_{\mu \nu}^{*}\right),
$$

and Einstein's equations (3.7a) can now be solved straightforwardly, still to lowest order around a flat Minkowski background. In particular, one may write $\left(8 \pi G / c^{4}\right)\left(T_{00}^{* \varphi}-\frac{1}{2} g_{00}^{*} T_{\varphi}^{*}\right)=$ $R_{00}^{*} \approx \frac{1}{2} \Delta \ln \left(-g_{00}^{*}\right)$, and this gives immediately

$$
-g_{00}^{*}=1-\frac{2 G M}{r c^{2}}-\frac{(n-1)(2 n-1)^{2}}{n(2 n-3)} \frac{\alpha G M}{\ell_{0} c^{2}}\left(\frac{2 \alpha G M \ell_{0}}{n r^{2} c^{2}}\right)^{1 /(2 n-1)}+\mathcal{O}\left(\frac{1}{c^{4}}\right) .
$$


The $R_{r r}^{*}$ component of Einstein's equations can now be used to solve for $g_{r r}^{*}$, but one must specify the coordinate system to write the solution explicitly. In order to compute easily the light deflection angle below, it will be convenient to use the so-called "Schwarzschild coordinates" (which define an "area radius" $r$ ), such that the line element takes the form

$$
d s_{*}^{2}=-\mathcal{B}(r) c^{2} d t^{2}+\mathcal{A}(r) d r^{2}+r^{2}\left(d \theta^{2}+\sin ^{2} \theta d \phi^{2}\right) .
$$

One then finds straightforwardly

$$
g_{r r}^{*}=\mathcal{A}(r)=1+\frac{2 G M}{r c^{2}}+\frac{\alpha G M}{n \ell_{0} c^{2}}\left(\frac{2 \alpha G M \ell_{0}}{n r^{2} c^{2}}\right)^{1 /(2 n-1)}+\mathcal{O}\left(\frac{1}{c^{4}}\right) .
$$

The physical metric $\tilde{g}_{\mu \nu}=e^{2 \alpha \varphi} g_{\mu \nu}^{*}$ to which matter is coupled can now be deduced from Eqs. (3.9), (3.11) and (3.13). In particular, the gravitational potential felt by test masses reads $V=-\frac{1}{2}\left(1+\tilde{g}_{00}\right) c^{2}$, and its radial derivative $\partial_{r} V$ gives their acceleration

$$
a=\frac{G M}{r^{2}}+\left(\frac{2 \alpha G M \ell_{0}}{n r^{2} c^{2}}\right)^{1 /(2 n-1)}\left[\frac{\alpha c^{2}}{\ell_{0}}+\left(1-\frac{1}{n}\right)\left(1+\frac{2}{2 n-3}\right) \frac{\alpha G M}{\ell_{0} r}\right]
$$

For large enough values of the integer $n$, this model predicts thus a MOND-like acceleration $\propto 1 / r$, together with the standard Newtonian one $G M / r^{2}$ and a constant contribution $\alpha c^{2} / \ell_{0}$. This constant contribution comes from the conformal factor $A^{2}(\varphi)$ entering the physical metric, i.e., from a scalar exchange between the massive source $M$ and the test mass, as in line (a) of Fig. 5 above. The standard Newtonian force comes from the curvature of the Einstein metric $g_{\mu \nu}^{*}$ caused by the matter stress-energy tensor. On the other hand, the $1 / r$ force comes from the curvature of $g_{\mu \nu}^{*}$ caused by the stress-energy tensor of the scalar field, as illustrated in line (c) of Fig. 5. However, let us underline that this model does not reproduce the MOND dynamics, first because the constant contribution happens to dominate over the $1 / r$ force, but above all because the coefficient of this $1 / r$ force is proportional to the baryonic mass $M$ instead of $\sqrt{M}$.

In order to compute the light deflection angle predicted by such a model, let us use its exact integral expression [117]

$$
\Delta \theta=-\pi+2 \int_{r_{0}}^{\infty} \frac{d r}{r^{2}}\left(\frac{\mathcal{A}(r) \mathcal{B}(r)}{\mathcal{B}\left(r_{0}\right) / r_{0}^{2}-\mathcal{B}(r) / r^{2}}\right)^{1 / 2}
$$

valid for any metric expressed in Schwarzschild coordinates (3.12). In this integral (3.15), $r_{0}$ denotes the smallest distance between the light ray and the center of the deflecting body, which may also be replaced by the impact parameter $b=r_{0}+\mathcal{O}\left(1 / c^{2}\right)$ at lowest order. Actually, since the coordinates we chose to write Eq. (3.13) are such that the Einstein metric $g_{\mu \nu}^{*}$ takes the Schwarzschild form, this is no longer the case for the physical metric $\tilde{g}_{\mu \nu}=A^{2}(\varphi) g_{\mu \nu}^{*}$, because the radial dependence of the scalar field (3.9) spoils the exact expressions $g_{\theta \theta}^{*}=r^{2}$ and $g_{\phi \phi}^{*}=r^{2} \sin ^{2} \theta$. However, we saw in Sec. IIIA that light is totally insensitive to any global factor of the metric, so that one may actually compute its deflection directly from the Einstein metric $g_{\mu \nu}^{*}$. The anomalous terms in Eqs. (3.11) and (3.13) are of the form

$$
\mathcal{A}(r)=1+\frac{k_{\mathcal{A}}}{r^{p}}, \quad \mathcal{B}(r)=1+\frac{k_{\mathcal{B}}}{r^{p}},
$$


where $p \equiv 2 /(2 n-1)=\mathcal{O}(1 / n)$ is a small number, and where $k_{\mathcal{A}}=\mathcal{O}(1 / n)$ whereas $k_{\mathcal{B}}=\mathcal{O}(n)$. For any value of these coefficients, integral (3.15) gives straightforwardly

$$
\Delta \theta=\left(\frac{k_{\mathcal{A}}}{p}-k_{\mathcal{B}}\right) \frac{\sqrt{\pi} \Gamma\left(\frac{1+p}{2}\right)}{\Gamma(p / 2)} \frac{1}{b^{p}}+\mathcal{O}\left(k_{\mathcal{A}, \mathcal{B}}^{2}\right),
$$

and in our present case

$$
\begin{aligned}
\Delta \theta & =\Delta \theta_{\mathrm{GR}}+\frac{\pi}{2} \frac{k_{\mathcal{A}}-p k_{\mathcal{B}}}{b^{p}}+\mathcal{O}\left(k_{\mathcal{A}, \mathcal{B}}^{2}\right)+\mathcal{O}\left(p^{2}\right) \\
& =\frac{4 G M}{b c^{2}}+\frac{\pi \alpha G M}{\ell_{0} c^{2}}\left(\frac{2 \alpha G M \ell_{0}}{n b^{2} c^{2}}\right)^{1 /(2 n-1)}\left[1+\mathcal{O}\left(\frac{1}{n}\right)\right]+\mathcal{O}\left(\frac{1}{c^{4}}\right) .
\end{aligned}
$$

It is interesting to note that in Eq. (3.18a), $p k_{\mathcal{B}}=\mathcal{O}\left(n^{0}\right)$ dominates over $k_{\mathcal{A}}=\mathcal{O}\left(n^{-1}\right)$, in spite of its small factor $p$. For large values of $n$, the anomalous contribution $\pi \alpha G M / \ell_{0} c^{2}$ to the deflection angle (3.18b) is precisely half the result GR would have predicted in presence of pressureless dark matter reproducing the $1 / r$ acceleration in (3.14) [namely, $T_{00}^{\text {dark }}=\alpha M c^{2} / 4 \pi \ell_{0} r^{2}$ and $\left.T_{\mu i}^{\text {dark }}=0\right]$. This is due to the specific form of the scalar stressenergy tensor (3.10), whose spatial components $T_{i j}^{* \varphi}$ do not vanish. But besides this subtle factor 2 discrepancy with the standard dark matter prediction, let us also recall that light is insensitive to the conformal factor $A^{2}(\varphi)$, responsible for the dominant anomalous contribution in (3.14), namely the constant one $\alpha c^{2} / \ell_{0}$. In conclusion, using the notation introduced in Sec. IIIA, the present model actually predicts $M_{\text {tot }}^{\text {lens }} \ll M_{\text {tot }}^{\text {rot }}$, which is even worse than the inequality (3.5) derived in standard scalar-tensor theories. On the other hand, for a large enough impact parameter $b$, the light deflection angle (3.18b), $\Delta \theta \approx \pi \alpha G M / \ell_{0} c^{2}=$ const., is much larger than the one predicted by GR in presence of $M$ alone, namely $4 G M / b c^{2} \rightarrow 0$. Therefore, we have exhibited a RAQUAL model predicting $M_{\mathrm{tot}}^{\text {lens }} \gg M_{\mathrm{b}}$.

One might try to refine the model (3.6) by choosing another matter-scalar coupling function, for instance

$$
A(\varphi)=\exp \left(\alpha \varphi^{-2 n}\right)
$$

with the same integer $n$ as in the kinetic term of the scalar field. A first useful consequence of such a choice is that the extra constant acceleration we found in Eq. (3.14) above becomes now negligible. Indeed, it came from the conformal factor $A^{2}(\varphi)$ relating the physical and Einstein metrics, so that this extra acceleration derives now from $\alpha \varphi^{-2 n} c^{2}=\mathcal{O}\left(r^{-2 n}\right)$, and tends quickly towards 0 as $r$ grows. On the other hand, the $1 / r$ acceleration of Eq. (3.14) is still predicted, since it comes from the curvature of the Einstein metric caused by the scalar's stress-energy tensor $\propto 1 / r^{2}$. For large enough impact parameters, such a model predicts thus

$$
M_{\mathrm{tot}}^{\text {lens }} \approx \frac{1}{2} M_{\mathrm{tot}}^{\mathrm{rot}} \gg M_{\mathrm{b}}
$$

the factor $\frac{1}{2}$ coming from the same calculation as Eq. (3.18) above. The second consequence of the choice (3.19) is that Eq. (3.7b) now involves the non-constant coefficient $d \ln A(\varphi) / d \varphi=-2 n \alpha / \varphi^{2 n+1}$. If one naively counts the powers of $\partial_{r} \varphi$ and $\varphi$ entering this Eq. (3.7b), one could then deduce that $\varphi^{4 n} \propto T_{\text {matter }}^{*} \propto M$. Using now Eq. (3.8), this would imply $T_{\mu \nu}^{* \varphi} \propto\left(\partial_{r} \varphi\right)^{2 n} \propto \sqrt{M}$, which looks like the dark matter MOND wishes to mimic. However, this would be the case only if $\varphi \rightarrow 0$ as $r \rightarrow 0$, within body $M$. Then one can prove that $\varphi \propto \sqrt{r}$ for small radii, and $\int d^{3} x T_{\mu \nu}^{* \varphi}$ would diverge within the body. Therefore, 
such a solution is unphysical. On the other hand, if $\varphi \rightarrow \varphi_{0} \neq 0$ within the body, then one gets back an exterior solution of the form (3.9), where $\alpha$ is replaced here by $-2 n \alpha / \varphi_{0}^{2 n+1}$, so that the anomalous force is a priori again proportional to $M$ instead of the looked-for $\sqrt{M}$. There could still remain a possibility to recover the MOND force with such a RAQUAL model, if one could prove that $\varphi \rightarrow \varphi_{0} \propto M^{1 / 4 n}$ within the body. This would be the case for instance if $\varphi \rightarrow 0$ at a given radius, say at the surface of the body. However, this would correspond to a diverging matter-scalar coupling strength at this radius (singular and obviously forbidden by solar-system tests), and we anyway do not see how to impose such a relation, nor the precise value of $\varphi_{0}$. In standard scalar-tensor theories, the scalar field tends towards a constant $\varphi_{0}$ when $r \rightarrow \infty$, this constant being imposed by the cosmological evolution of the Universe, and being constrained by post-Newtonian tests of gravity in the solar system. Here, the scalar field $\varphi=\varphi_{0}+$ const. $r^{1-2 /(2 n-1)}$ diverges at infinity, as soon as a single particle exists in the Universe. This pathological behavior is linked with the fact that the solution of Eq. (3.7b) cannot be written unambiguously, the integration constant $\varphi_{0}$ remaining undetermined. In the model (3.9), where $A(\varphi)=e^{\alpha \varphi}$, this constant did not enter any physical observable, but it does when the matter-scalar coupling function is no longer a mere exponential, as in our present attempt (3.19). Therefore, although this matter-scalar coupling function looks promising to reproduce the MOND dynamics from a simple RAQUAL model, it fails to define a fully predictive theory. Its only solid prediction is that light bending would be twice smaller than the prediction of GR in presence of dark matter, cf. Eq. (3.20). As far as we know, this is still allowed by weak-lensing observations [114, 115, 116].

In conclusion, we have exhibited RAQUAL models predicting a much larger light deflection than GR in presence of baryonic matter alone, but this framework does not seem to reproduce the MOND dynamics consistently. Note that the class of RAQUAL theories examined in the present subsection IIIB are actually models of dark matter. Extra Newtonian force and light deflection are caused by the stress-energy tensor of the scalar field, which plays thus the role of dark matter. The only difference with standard dark matter models is that its distribution is strictly imposed by the one of baryonic matter, whereas they are usually only related via the dynamical evolution of large-scale structures.

\section{Disformal coupling}

A nice trick exists to increase light deflection within scalar-tensor theories or RAQUAL models. It is similar to Ni's "stratified theory of gravity" [1, 118], and was introduced by Bekenstein in [28]; see also [29]. Instead of assuming that matter is coupled to a physical metric $\tilde{g}_{\mu \nu}=A^{2}(\varphi) g_{\mu \nu}^{*}$, conformally related to the Einstein metric $g_{\mu \nu}^{*}$, one may consider a "disformal" relation of the form

$$
\tilde{g}_{\mu \nu}=A^{2}(\varphi, s) g_{\mu \nu}^{*}+B(\varphi, s) \partial_{\mu} \varphi \partial_{\nu} \varphi
$$

where as before $s \equiv g_{*}^{\mu \nu} \partial_{\mu} \varphi \partial_{\nu} \varphi$. The crucial difference is that the $\partial_{\mu} \varphi \partial_{\nu} \varphi$ contribution now privileges a particular direction, namely the radial one in spherically symmetrical systems. This extra contribution may remain negligible in the equations of motion of test particles, but now light can be directly sensitive to the presence of the scalar field, contrary to the case of conformal coupling that we discussed in Sec. IIIA.

Let us emphasize that because of the disformal coupling above, light (or matter) can travel faster or slower than gravitons depending on the sign of $B$. In a similar way as in 
Sec. IIE, this does not imply a violation of causality, provided that the matter metric is still of Lorentzian signature. The matter metric Eq. (3.21) is causal if

$$
A^{2}(\varphi, s)>0 \text { and } A^{2}(\varphi, s)+s B(\varphi, s)>0
$$

for all $\varphi$ and $s$. Notice that if $A$ does not depend on $s, B$ must depend on $s$ for the latter inequality to hold. Beware that these kind of models used in the literature as realizations of varying speed of light scenarios [119, 120] do not respect this last inequality. Indeed, whereas $A$ was taken to be $1, B$ was chosen constant $B=-L^{2}$ with $L$ being some fixed length scale. Therefore matter field equations are elliptic at local scales $\left(s>1 / L^{2}\right)$, and do not have a well-posed Cauchy problem.

By analogy with the Schwarzschild metric, whose positive contribution in the radial component $g_{r r}=1+2 G M / r c^{2}+\mathcal{O}\left(1 / c^{4}\right)$ contributes positively to light deflection (together with the standard Newtonian potential involved in $\left.g_{00}\right)$, one understands intuitively that positive values of $B(s, \varphi)$ should increase light deflection. To prove it, let us first recall again that light is insensitive to any global factor of the metric, because of the conformal invariance of electromagnetism in 4 dimensions, Eq. (3.1). Therefore, instead of the metric (3.21), one may equivalently consider $\bar{g}_{\mu \nu} \equiv g_{\mu \nu}^{*}+\left(B / A^{2}\right) \partial_{\mu} \varphi \partial_{\nu} \varphi$. Let us then assume that the coordinates have been chosen so that the Einstein metric $g_{\mu \nu}^{*}$ takes the Schwarzschild form (3.12). Since, in a static and spherically symmetric situation, the disformal contribution $\left(B / A^{2}\right) \partial_{\mu} \varphi \partial_{\nu} \varphi$ only contributes to the radial component $\bar{g}_{r r}$, the metric $\bar{g}_{\mu \nu}$ is also of the Schwarzschild form, and one may thus use integral (3.15) to compute the light deflection angle. The only difference with $\mathrm{GR}$ is that the radial metric component now reads $\mathcal{A}=$ $\mathcal{A}_{\mathrm{GR}}+\left(B / A^{2}\right)\left(\partial_{r} \varphi\right)^{2}$. [Beware not to confuse the metric components $\mathcal{A} \equiv \bar{g}_{r r}$ and $\mathcal{B} \equiv-\bar{g}_{00}$ defined in (3.12) with the scalar-field functionals $A(s, \varphi)$ and $B(s, \varphi)$ entering (3.21).] At first post-Newtonian order, all contributions coming from $g_{\mu \nu}^{*}$ reproduce the general relativistic result, and the only extra contribution is thus the one proportional to $\left(B / A^{2}\right)\left(\partial_{r} \varphi\right)^{2}$, assumed to be of order $\mathcal{O}\left(1 / c^{2}\right)$ too:

$$
\Delta \theta=\Delta \theta_{\mathrm{GR}}+\int_{r_{0}}^{\infty} \frac{d r / r}{\sqrt{r^{2} / r_{0}^{2}-1}} \frac{B\left(\partial_{r} \varphi\right)^{2}}{A^{2}}+\mathcal{O}\left(\frac{1}{c^{4}}\right) .
$$

Even without performing the explicit integration in particular models, it is thus clear that a positive value of $B(s, \varphi)$ always gives a positive contribution to light bending, since the extra term in (3.23) is the integral of a positive function.

For instance, if one tunes the model such that $\left(B / A^{2}\right)\left(\partial_{r} \varphi\right)^{2}=4 \sqrt{G M a_{0}} / c^{2}$ is independent of $r$ (as we will do in Sec. $\mathrm{V}$ below), then

$$
\Delta \theta=\Delta \theta_{\mathrm{GR}}+\frac{2 \pi \sqrt{G M a_{0}}}{c^{2}}+\mathcal{O}\left(\frac{1}{c^{4}}\right)
$$

and the second term reproduces exactly the deflection GR would have predicted in presence of dark matter (which is independent of the impact parameter, ${ }^{10}$ as if there were a

10 Note that although the deflection angle is constant, the direction of deflection is radial with respect to the deflecting body, so that distant objects are deformed and weak lensing observations can be performed. A constant deflection angle in a constant direction would have just shifted globally the image of the distant objects, without any deformation. 
deficit angle in a flat conical space). Indeed, it is straightforward to prove that a dark matter density $T_{00} / c^{2}=\sqrt{G M a_{0}} / 4 \pi G r^{2}$ generates a logarithmic MOND-like potential in the time component of the metric, $-\tilde{g}_{00}=1-2 G M / r c^{2}+2 \sqrt{G M a_{0}} \ln r / c^{2}+\mathcal{O}\left(1 / c^{4}\right)$, and that the corresponding radial metric reads $\tilde{g}_{r r}=1+2 G M / r c^{2}+2 \sqrt{G M a_{0}} / c^{2}+\mathcal{O}\left(1 / c^{4}\right)$ in Schwarzschild coordinates. A similar calculation as above then yields the same light deflection angle (3.24) [see Eq. (3.32) below for an explicit derivation]. Note in passing that $\tilde{g}_{r r} \neq-1 / \tilde{g}_{00}$ in such coordinates, contrary to the standard Schwarzschild solution in vacuum. On the other hand, if one rewrites this same metric in isotropic coordinates, $d \tilde{s}^{2}=-\tilde{g}_{00} c^{2} d t^{2}+\tilde{g}_{\rho \rho}\left(d \rho^{2}+\rho^{2} d \theta^{2}+\rho^{2} \sin ^{2} \theta d \phi^{2}\right)$, one indeed recovers the usual relation $\tilde{g}_{\rho \rho}=-1 / \tilde{g}_{00}+\mathcal{O}\left(1 / c^{4}\right)=1+2 G M / \rho c^{2}-2 \sqrt{G M a_{0}} \ln \rho / c^{2}+\mathcal{O}\left(1 / c^{4}\right)$. This remark recalls us that a metric is coordinate-dependent, and that observable quantities cannot be read naively in its coefficients. Even the signs of the predicted effects are not obvious. For instance, if one had naively tried to impose $A^{2}(s, \varphi)=\left(1+2 \sqrt{G M a_{0}} \ln r / c^{2}\right)$ but $\tilde{g}_{r r}=-1 / \tilde{g}_{00}$ in Eq. (3.21), then one would have needed a negative $B(s, \varphi)$, and the predicted deflection angle would have been negative (and diverging), $\Delta \theta=\Delta \theta_{\mathrm{GR}}-2 \pi \sqrt{G M a_{0}} \ln 2 b / c^{2}$.

Equation (3.23) proves that a positive value of $B(s, \varphi)$ always increases light deflection. However, light rays satisfy then $\tilde{g}_{\mu \nu} d x^{\mu} d x^{\nu}=0 \Leftrightarrow g_{\mu \nu}^{*} d x^{\mu} d x^{\nu}=-B / A^{2}\left(\partial_{\mu} \varphi d x^{\mu}\right)^{2} \leq 0$, and photons are thus timelike with respect to the Einstein metric $g_{\mu \nu}^{*}$. This has been considered as a serious flaw by the authors of Refs. [28, 29]. Indeed, this means that gravitons (i.e., perturbations of the Einstein metric $g_{\mu \nu}^{*}$ ) are superluminal, and it was believed that this implied causality violations. References [29] even present the reasoning the other way: If one imposes from the beginning that no field (even gravity) can propagate faster than light, then a disformal coupling (3.21) needs $B \leq 0$, therefore light deflection is even smaller that the one predicted in standard scalar-tensor or RAQUAL models, see Sec. IIIA. This sufficed for the authors of these references to discard such models. Actually, as already discussed in Sec. IIE, such a superluminal propagation does not threaten causality. This is even clearer in the present case, since one is used to write the field equations in terms of the Einstein metric $g_{\mu \nu}^{*}$ (i.e., in what is usually called the "Einstein frame"), in order to diagonalize the kinetic terms and prove that the Cauchy problem is well posed. To guarantee causality, like in GR, one needs to assume that the topology of spacetime does not involve any closed timelike curve with respect to $g_{\mu \nu}^{*}$. Since the causal cone of matter, defined by the physical metric $\tilde{g}_{\mu \nu}$, is always interior to the one defined by $g_{\mu \nu}^{*}$ if $B>0$, there cannot exist any CTC for any field. In conclusion, the nice trick of disformal coupling, Eq. (3.21), is consistent from the point of view of causality.

It remains however to study its consistency within matter. Indeed, the physical metric $\tilde{g}_{\mu \nu}$ is involved in many places of the matter action, to contract indices, to define covariant derivatives, and in the volume element $\sqrt{-\tilde{g}} d^{4} x$. One should thus take into account the matter contribution $\delta S_{\text {matter }}\left[\psi ; \tilde{g}_{\mu \nu}\right] / \delta \varphi$ in the Euler-Lagrange equation (2.14) for $\varphi$, and study simultaneously the matter field equations $\delta S_{\text {matter }}\left[\psi ; \tilde{g}_{\mu \nu}\right] / \delta \psi=0$ (where as before $\psi$ denotes any matter field). One should then identify any second derivative of $\varphi$ which appears in them, and prove that the full set of field equations, for matter, the scalar field and the Einstein metric $g_{\mu \nu}^{*}$, has a well-posed Cauchy problem. This is usually done by diagonalizing the kinetic terms, i.e., by separating all second derivatives in the left-hand side of the field equations, and by proving that their differential operator is hyperbolic. In the general case of a theory involving any kind of matter, this is quite involved and we postpone this crucial study to a future work. Indeed, if one considers an action of the form (2.13) with a physical 
metric (3.21), the scalar field equation reads

$$
\begin{aligned}
& \sqrt{-g^{*}} \nabla_{\mu}^{*}\left[\frac{\partial f}{\partial s} \nabla_{*}^{\mu} \varphi\right] \\
& -\frac{4 \pi G}{c^{4}} \partial_{\mu}\left\{\sqrt{-\tilde{g}}\left[B \tilde{T}^{\mu \nu}+2 \frac{\partial \ln A}{\partial s} \tilde{T} g_{*}^{\mu \nu}+\left(\frac{\partial B}{\partial s}-2 B \frac{\partial \ln A}{\partial s}\right) \tilde{T}^{\rho \sigma} \partial_{\rho} \varphi \partial_{\sigma} \varphi g_{*}^{\mu \nu}\right] \partial_{\nu} \varphi\right\} \\
& =\sqrt{-g^{*}}\left(\frac{1}{2} \frac{\partial f}{\partial \varphi}+\frac{\partial V}{\partial \varphi}\right)-\frac{4 \pi G}{c^{4}} \sqrt{-\tilde{g}}\left[\frac{\partial \ln A}{\partial \varphi} \tilde{T}+\left(\frac{1}{2} \frac{\partial B}{\partial \varphi}-B \frac{\partial \ln A}{\partial \varphi}\right) \tilde{T}^{\rho \sigma} \partial_{\rho} \varphi \partial_{\sigma} \varphi\right]
\end{aligned}
$$

where $\tilde{T}^{\mu \nu} \equiv(2 c / \sqrt{-\tilde{g}}) \delta S_{\text {matter }} / \delta \tilde{g}_{\mu \nu}$ is the physical stress-energy tensor, $\tilde{T} \equiv \tilde{T}^{\mu \nu} \tilde{g}_{\mu \nu}$, and $\tilde{g}=A^{8}\left(1+s B / A^{2}\right) g_{*}$ from definition (3.21). Note that Eq. (3.25) reduces to Eq. (2.15) for $A=e^{\alpha \varphi}$ and $B=0$. The right hand side of the above equation does not involve any second derivative and is merely a source term [if $\partial A / \partial \varphi$ were vanishing, $\partial_{\mu} \varphi=0$ would always be a solution]. The left hand side however contains second derivatives of the scalar field and of matter fields, through terms like $\partial \tilde{T}$. The equation is not diagonal and, moreover, the effective metric multiplying the second derivative of $\varphi$ has a rather complicated expression that notably involves terms like $\tilde{T}^{\mu \nu}, \tilde{T}^{\rho \sigma} \partial_{\rho} \varphi \partial_{\sigma} \varphi$. The Lorentzian signature of the latter is therefore a quite involved question, even if we expect that it may depend on generic energy conditions in the matter sector. Note that the literature on such MOND-like field theories did not discuss the consistency of the field equations within matter. We will not examine either the fully general case in the present paper, but we wish to underline that this should be done. We will however analyze below the particular case where matter is described by a pressureless and isentropic perfect fluid. Although much simpler than the general case, it is relevant for most matter in the universe, and exhibits the subtle conditions which must be imposed on the coupling constants $A$ and $B$ for the scalar field equation to remain always consistent. As far as we are aware, this is the first time such a discussion is presented.

The action defining a pressureless and isentropic perfect fluid reads $S_{\text {matter }}=$ $-\int d^{4} x \sqrt{-\tilde{g}} \tilde{\rho} c$, where $\tilde{\rho}$ is the conserved matter density as observed in the Jordan frame. If $\tilde{u}^{\lambda} \equiv d x^{\lambda} / \sqrt{-\tilde{g}_{\mu \nu} d x^{\mu} d x^{\nu}}$ denotes the matter unit 4-velocity, one has thus $\tilde{\nabla}_{\lambda}\left(\tilde{\rho} \tilde{u}^{\lambda}\right)=0$, which can be equivalently written in terms of a partial derivative as $\partial_{\lambda}\left(\sqrt{-\tilde{g}} \tilde{\rho} \tilde{u}^{\lambda}\right)=0$. This equation implies that the matter current density $\sqrt{-\tilde{g}} \tilde{\rho} \tilde{u}^{\lambda}$ is determined everywhere once initial conditions are given at a point of the flowlines, and it is therefore unchanged if the metric $\tilde{g}_{\mu \nu}$ is varied: $\delta\left(\sqrt{-\tilde{g}} \tilde{\rho} \tilde{u}^{\lambda}\right) / \delta \tilde{g}_{\mu \nu}=0$. In particular, the coordinate-conserved density $^{11} \bar{\rho} \equiv \sqrt{-\tilde{g}} \tilde{\rho} \tilde{u}^{0}$ is independent of the Jordan metric $\tilde{g}_{\mu \nu}$, and thereby independent of both the Einstein metric $g_{\mu \nu}^{*}$ and the scalar field $\varphi$ (as well as its derivatives $\partial_{\mu} \varphi$ ). The pressureless perfect fluid action may thus be written as $S_{\text {matter }}=-\int d^{4} x \bar{\rho} c / \tilde{u}^{0}=$ $-\int d^{3} x \bar{\rho} c \sqrt{-\tilde{g}_{\mu \nu} d x^{\mu} d x^{\nu}}$, which does depend explicitly on the scalar field via the Jordan metric (3.21), and therefore contributes to the scalar-field dynamics within matter. Introducing the Einstein-frame unit 4-velocity $u_{*}^{\lambda} \equiv d x^{\lambda} / \sqrt{-g_{\mu \nu}^{*} d x^{\mu} d x^{\nu}}$ (independent of $\varphi$ and

11 The coordinate-conserved density $\bar{\rho}$ is often denoted as $\rho^{*}$ in the general relativistic literature, but this star index would be confusing in our present context where Einstein-frame quantities already bear such an index. 
its derivatives), one may thus write the action of the scalar field as

$$
S_{\varphi}=-\frac{c^{4}}{8 \pi G} \int \frac{d^{4} x}{c} \sqrt{-g_{*}}[f(s, \varphi)+2 V(\varphi)]-\int \frac{d^{4} x}{c} \frac{\bar{\rho} c^{2}}{u_{*}^{0}} \sqrt{A^{2}(\varphi, s)-\left(u_{*}^{\mu} \partial_{\mu} \varphi\right)^{2} B(\varphi, s)},
$$

where all the scalar-field dependences are now explicitly exhibited (neither $\sqrt{-g_{*}}$ nor $\bar{\rho} c^{2} / u_{*}^{0}$ depend on it). In conclusion, the dynamics of the scalar field within matter is described by an action generalizing the RAQUAL form (2.13), in which the function $f(\varphi, s)$ is replaced by

$$
\tilde{f}\left(\varphi, s, u_{*}^{\mu} \partial_{\mu} \varphi\right)=f(\varphi, s)+\frac{8 \pi G \bar{\rho}}{\sqrt{-g_{*}} u_{*}^{0} c^{2}} A \sqrt{1-\left(u_{*}^{\mu} \partial_{\mu} \varphi\right)^{2} B / A^{2}},
$$

where $A(\varphi, s)$ and $B(\varphi, s)$ are themselves functions of the scalar field and $s \equiv g_{*}^{\mu \nu} \partial_{\mu} \varphi \partial_{\nu} \varphi$. Since the derivatives of the scalar field do not enter only via $s$ but also via $u_{*}^{\mu} \partial_{\mu} \varphi$, the conditions that such a function $\tilde{f}$ must satisfy to define a consistent scalar-field equation (hyperbolicity and Hamiltonian bounded by below) are significantly more complicated than (a) and (b) of Sec. IIE above [that $f$ itself must still satisfy to define a consistent scalar-field equation in vacuum]. Using as before a prime to denote partial derivation with respect to $s$, i.e., $\tilde{f}^{\prime} \equiv \partial \tilde{f}\left(\varphi, s, u_{*}^{\mu} \partial_{\mu} \varphi\right) / \partial s$, and introducing the shorthand notation $\hat{\rho} \equiv 2 \pi G \bar{\rho} / \sqrt{-g_{*}} u_{*}^{0} c^{2}$, $C \equiv B / \sqrt{A^{2}-\left(u_{*}^{\mu} \partial_{\mu} \varphi\right)^{2} B}$, and $D \equiv C+\left(u_{*}^{\mu} \partial_{\mu} \varphi\right)^{2} C^{3} / B$, the hyperbolicity conditions for the scalar field equation within matter may be written as

$$
\begin{aligned}
& \text { (a1) } \tilde{f}^{\prime}>0, \\
& \text { (b1) } s \tilde{f}^{\prime \prime}+\tilde{f}^{\prime}+\hat{\rho} D-4 \hat{\rho}\left(u_{*}^{\mu} \partial_{\mu} \varphi\right)^{2} C^{\prime} \\
& \quad \pm\left\{\left(s \tilde{f}^{\prime \prime}-\hat{\rho} D\right)^{2}-4 \hat{\rho}\left(u_{*}^{\mu} \partial_{\mu} \varphi\right)^{2}\left(\tilde{f}^{\prime \prime}+2 \hat{\rho} C^{\prime}\right)\left(2 s C^{\prime}+D\right)\right\}^{1 / 2}>0 .
\end{aligned}
$$

Note that in condition (b1), the inequality must be satisfied for both signs of the square root, and it suffices thus to check its lowest value, involving a minus sign. At the lowest post-Newtonian level, in the limit $\left(u_{*}^{\mu} \partial_{\mu} \varphi\right)^{2} \ll|s|$, one may simplify these conditions as

(a2) $\tilde{f}^{\prime}>0$,

(b2) $2 s \tilde{f}^{\prime \prime}+\tilde{f}^{\prime}>0$

(c2) $\tilde{f}^{\prime}+2 \hat{\rho} C>0$.

If $B$ is chosen positive in Eq. (3.21), in order to increase light deflection in the MOND regime, then $\hat{\rho} C>0$ and condition (c2) is implied by (a2). On the other hand, in the ultrarelativistic regime, or in the cosmological one where $\left|\partial_{0} \varphi\right| \gg\left|\partial_{i} \varphi\right|$, conditions (a1) and (b1) reduce to

(a3) $\tilde{f}^{\prime}>0$

(b3) $2 s \tilde{f}^{\prime \prime}+\tilde{f}^{\prime}+2 \hat{\rho}\left(4 s C^{\prime}+D\right)>0$. 
Note however that conditions (a1) and (b1) should in principle be satisfied in any situation (within matter), in order for the model to always remain a well-defined field theory.

In addition to (a1) and (b1), the contribution of the scalar field to the Hamiltonian should also be bounded by below. In the rest frame of the perfect fluid, such that $u_{*}^{\mu}=(1,0,0,0)$, and simultaneously with coordinates which diagonalize locally the Einstein metric $g_{\mu \nu}^{*}=$ $\operatorname{diag}(-1,1,1,1)$, this contribution is proportional to $\tilde{f}+2\left(\partial_{0} \varphi\right)^{2}\left(\tilde{f}^{\prime}+2 \hat{\rho} C\right)$. The general conditions needed to ensure that it is bounded by below are more involved. It suffices to check that this is indeed the case for each particular model that one may consider.

Let us finally mention that no extra condition is necessary to ensure that the argument of the square root is positive in Eqs. (3.26)-(3.27). Indeed, since matter is assumed to be metrically coupled to the Jordan metric $\tilde{g}_{\mu \nu}$, we know that $\tilde{g}_{\mu \nu} \tilde{u}^{\mu} \tilde{u}^{\nu}=-1$, i.e., that the matter 4 -velocity must be timelike with respect to $\tilde{g}_{\mu \nu}$. This implies $1-\left(u_{*}^{\mu} \partial_{\mu} \varphi\right)^{2} B / A^{2}>0$, which should be understood as a condition (automatically) satisfied by the Einstein-frame 4 -velocity $u_{*}^{\mu}$, but not as a constraint on the functions $A(\varphi, s)$ and $B(\varphi, s)$.

Besides this important question of the consistency of the scalar field equation within matter, it also remains to study the precise predictions of this class of models. Specific examples have been analyzed in Refs. [28, 29, 119, 120]. We will examine a very different one in Sec. VB below, but still within this class of disformally coupled scalar-tensor theories. We will notably show that the predicted light deflection can be consistent both with weaklensing determinations of dark matter and with post-Newtonian tests in the solar system.

\section{Stratified theories}

Because the above superluminal propagation of gravity was considered as deadly, Sanders proposed in [32, 33] another kind of disformal coupling, where the physical metric to which matter is universally coupled takes the form

$$
\tilde{g}_{\mu \nu}=A^{2}(\varphi) g_{\mu \nu}^{*}+B(\varphi) U_{\mu} U_{\nu}
$$

This is thus strictly the same form as Eq. (3.21) above, but the gradient $\partial_{\mu} \varphi$ has been replaced by a vector field $U_{\mu}$ (not to be confused with the matter unitary velocities $\tilde{u}^{\mu}$ and $u_{*}^{\mu}$ introduced in Sec. IIIC above). Bekenstein's theory TeVeS [30, 31] also assumes the above disformal relation. The dynamics of the vector field is defined by a specific kinetic term of the Proca form $\left(\partial_{[\mu} U_{\nu]}\right)^{2}$, and its norm is imposed to be $g_{*}^{\mu \nu} U_{\mu} U_{\nu}=-1$ thanks to a Lagrange parameter. The reason why it is necessary to fix its norm is because the magnitude of scalar-field effects would otherwise depend on the background norm of the vector field. Since backgrounds where $U_{\mu}=0$ would be possible, one would not predict any extra light deflection caused by the scalar field in such situations. Moreover the Lagrange multiplier ensures that the vector field equation is consistent with any type of matter one considers. Indeed, without the Lagrange multiplier, the equation of motion reads

$$
\nabla_{\mu}^{*}\left(\nabla_{*}^{[\mu} U_{*}^{\nu]}\right) \propto \frac{\sqrt{-\tilde{g}}}{\sqrt{-g_{*}}} \tilde{T}^{\rho \nu} U_{\rho}
$$

where $U_{*}^{\nu} \equiv g_{*}^{\mu \nu} U_{\mu}$, and its divergence imposes that the stress-energy tensor of matter must

satisfy the non-trivial property $\nabla_{\nu}^{*}\left(\sqrt{-\tilde{g}} / \sqrt{-g_{*}} \tilde{T}^{\rho \nu} U_{\rho}\right)=0$, that may be satisfied only by very particular type of matter. On the other hand, a term like $\lambda\left(U_{\mu} U_{*}^{\mu}+1\right)$ in the action, where $\lambda$ is a Lagrange multiplier, gives rise to a term proportional to $\lambda U_{*}^{\nu}$ in the left hand 
side of Eq. (3.29), whose divergence equals the a priori non vanishing divergence of the right hand side, thus giving its value to $\lambda$. Note finally that the consistency of the field equations requires that the above matter metric Eq. (3.28) has a Lorentzian signature. This condition reads $A^{2}>0$ and $A^{2}>B$ for all $\varphi$, where we used $U_{\mu} U_{*}^{\mu}=-1$.

One could think that the vector field is chosen timelike (negative norm), contrary to the gradient $\partial_{\mu} \varphi$ in the vicinity of clustered matter, in order to preserve isotropy of space which is very well tested experimentally. However, this is not the case, since a timelike vector does define a preferred frame (i.e., an "ether"), namely the one where it takes the canonical form $U_{\mu}=( \pm 1,0,0,0)$. We will briefly discuss the related experimental difficulties in Sec. IVB below.

The bonus added by this vector field $U_{\mu}$, with respect to the purely scalar case (3.21), is threefold: (i) it allows us to increase light deflection without necessitating superluminal propagation of gravity; (ii) it can also be used to tune a specific kinetic term for the scalar field, in order to avoid its own superluminal propagation identified in Sec. IIF above; (iii) the dynamics of the various fields is easier to analyze even within matter, and there does not seem to exist any generic difficulty with the Cauchy problem. Of course, the third point is actually the only useful one, since we know that superluminal propagation does not threaten causality provided there always exists a nonvanishing exterior to the union of all causal cones, as discussed in Sec. IIE above and in Ref. [103]. However, the real reason why this vector was considered in Refs. [30, 31, 32, 33] is indeed to avoid any superluminal propagation. Point (ii) is easy to understand: If the kinetic term of the scalar field is defined as $\left(g_{*}^{\mu \nu}-U_{*}^{\mu} U_{*}^{\nu}\right) \partial_{\mu} \varphi \partial_{\nu} \varphi$, instead of the standard $s=g_{*}^{\mu \nu} \partial_{\mu} \varphi \partial_{\nu} \varphi$, then a violation of condition (c) of Sec. IIE only implies that the scalar causal cone is exterior to the one defined by the inverse of $g_{*}^{\mu \nu}-U_{*}^{\mu} U_{*}^{\nu}$, namely $g_{\mu \nu}^{*}+\frac{1}{2} U_{\mu} U_{\nu}=\operatorname{diag}\left(-\frac{1}{2}, 1,1,1\right)$ in the preferred frame and in locally inertial coordinates. It becomes thus possible to reproduce the MOND dynamics while avoiding a propagation of $\varphi$ outside the causal cone defined by $g_{\mu \nu}^{*}=\operatorname{diag}(-1,1,1,1)$. Since point (i) is subtler, we discuss it now in more detail.

It is actually quite simple to understand why a coupling of matter (including electromagnetism) to the disformal metric (3.28) suffices to increase light deflection. The basic idea is similar to the case of the previous disformal coupling (3.21): Photons are now directly coupled to the scalar field, whereas they do not feel any global factor of the metric. More precisely, in the preferred frame where $U_{\mu}=( \pm 1,0,0,0)$, and in locally inertial coordinates such that $g_{\mu \nu}^{*}=\operatorname{diag}(-1,1,1,1)$ at a given spacetime point, one notices that $-U_{\mu} U_{\nu}=\operatorname{diag}(-1,0,0,0)$ behaves like the $g_{00}^{*}$ component of the metric, and conversely that $g_{\mu \nu}^{*}+U_{\mu} U_{\nu}=\operatorname{diag}(0,1,1,1)$ behaves like its spatial components $g_{i j}^{*}$. The vector field is thus a mere tool to separate by hand the space and time components of a tensor (implying obviously a violation of Lorentz invariance, namely the existence of an ether). Its interest is that one may now multiply $g_{00}^{*}$ and $g_{i j}^{*}$ by different functions of the scalar field. Inverse factors $e^{2 \alpha \varphi}$ and $e^{-2 \alpha \varphi}$ have been chosen in Refs. [30, 31, 32, 33], in order to mimic GR in isotropic coordinates. One could consider more generally inverse factors $A^{2}(\varphi)$ and $A^{-2}(\varphi)$, but an exponential (Brans-Dicke-like) coupling $A(\varphi)=e^{\alpha \varphi}$ suffices to predict the right phenomenology. The physical metric is thus chosen as

$$
\tilde{g}_{00}=e^{2 \alpha \varphi} g_{00}^{*} ; \quad \tilde{g}_{i j}=e^{-2 \alpha \varphi} g_{i j}^{*} .
$$

This can be rewritten covariantly thanks to the vector field $U_{\mu}$ defining the preferred frame:

$$
\begin{aligned}
\tilde{g}_{\mu \nu} & =-e^{2 \alpha \varphi} U_{\mu} U_{\nu}+e^{-2 \alpha \varphi}\left(g_{\mu \nu}^{*}+U_{\mu} U_{\nu}\right) \\
& =e^{-2 \alpha \varphi} g_{\mu \nu}^{*}-2 U_{\mu} U_{\nu} \sinh (2 \alpha \varphi) .
\end{aligned}
$$


This matter metric has a Lorentzian signature, as can be easily checked. Beware that our notations differ slightly from those of Refs. [30, 31]. In these references, the matter-scalar coupling constant $\alpha$ reads $\sqrt{k / 4 \pi}$ where $k$ is a dimensionless parameter.

In order to reproduce the MOND dynamics at large distances, the model must be tuned so that $e^{2 \alpha \varphi}=1+2 \sqrt{G M a_{0}} \ln r / c^{2}$ in this regime. Moreover, if one insists on the fact that photons should propagate faster than gravitons (even if it is actually not necessary), one must have $\alpha \varphi \geq 0$ at every spacetime point. The logarithm entering this MOND potential should therefore be understood as $\ln (r / \ell)$, where $\ell$ is a length scale significantly smaller than the size of the considered galaxy (either imposed dynamically by the model, or simply a universal constant much smaller than the size of any galaxy). Indeed, $d \tilde{s}^{2}=0 \Leftrightarrow d s_{*}^{2}=$ $2 e^{2 \alpha \varphi} \sinh (2 \alpha \varphi)\left(U_{\mu} d x^{\mu}\right)^{2} \geq 0$ because $\alpha \varphi \geq 0$, and the cone defined by $\tilde{g}_{\mu \nu}$ is thus wider than the one defined by $g_{\mu \nu}^{*}$.

Note that the character spacelike of $\partial_{\mu} \varphi$ or timelike of $U_{\mu}$ does not change the sign of the square $\left(\partial_{\mu} \varphi d x^{\mu}\right)^{2}$ or $\left(U_{\mu} d x^{\mu}\right)^{2}$. The crucial difference is that we now consider a negative factor for the term $U_{\mu} U_{\nu}$ entering the disformal metric (3.31), whereas we needed a positive value of $B(s, \varphi)$ to increase light bending with metric (3.21). In fact, it may seem paradoxical to increase light bending with the negative term entering Eq. (3.31), since photons now propagate faster than in GR (i.e., faster than gravitons), if $\alpha \varphi>0$. Intuitively, the trajectories of fast particles are almost straight lines, whereas slower ones are significantly curved by the Newtonian potential. This reasoning just happens to be erroneous, ${ }^{12}$ because light bending depends very differently on the temporal $\left(\tilde{g}_{00}\right)$ and spatial $\left(\tilde{g}_{i j}\right)$ components of the metric, as illustrated by Eq. (3.15) in Schwarzschild coordinates. At linear order, Ref. [29] actually proves that this integral involves the radial derivative $\partial_{r} \tilde{g}_{00}$ of the time component, thanks to a partial integration, whereas the radial component $\tilde{g}_{r r}$ enters it without any derivation. ${ }^{13}$ In other words, one needs an additional positive term in $\tilde{g}_{r r}$ to increase light bending, as proven in Eq. (3.23) above, but one needs a term whose radial derivative is negative if it enters $\tilde{g}_{00}$, as in Eq. (3.31) above. This is the reason why the Newtonian and MOND potentials enter so differently in the metric predicted by GR in presence of dark matter $\tilde{g}_{00}=-1+2 G M / r c^{2}-2 \sqrt{G M a_{0}} \ln r / c^{2}$ and $\tilde{g}_{r r}=1+2 G M / r c^{2}+2 \sqrt{G M a_{0}} / c^{2}$ [see below Eq. (3.24) ]. In spite of their relatives signs, all the above terms contribute positively to light bending, because those entering $\tilde{g}_{r r}$ are positive, and because the derivatives of those entering $\tilde{g}_{00}$ are negative.

The above discussion illustrates once again that observable quantities, or even their signs, can be subtle to identify in the components of a metric tensor. The only way to be sure of an observable prediction is to compute it explicitly. We already mentioned, below Eq. (3.24), that the GR metric in presence of dark matter takes precisely the form (3.30) in isotropic coordinates. Let us however derive now the light deflection angle it predicts. First of all, since light is insensitive to any global factor, let us multiply it by $e^{2 \alpha \varphi}$, i.e., consider instead

12 As an illustration of that point, let us recall that the scalar field surrounding a body of mass $M$ is not only given by $\varphi_{\text {local }}=-\alpha G M / r c^{2}$ (or $\sqrt{G M a_{0}} \ln r / \alpha c^{2}$, depending on the regime), but more generally by $\varphi_{0}+\varphi_{\text {local }}$ when one takes into account the asymptotic value of the scalar field $\varphi_{0}$ which evolves with cosmic time. Since the "speed" of photons (or, more precisely, their character timelike, null or spacelike with respect to the Einstein metric $g_{\mu \nu}^{*}$ ) depends on the sign of $\varphi_{0}+\varphi_{\text {local }}$, one may expect that light bending would be affected by $\varphi_{0}$. Actually this is not the case, as shown by the first line of Eq. (3.32).

13 This also explains why the time component $k_{\mathcal{B}}$ enters multiplied by a factor $-p$ in Eq. (3.18a), contrary to the radial component $k_{\mathcal{A}}$. 
the metric $\bar{g}_{00} \equiv e^{4 \alpha \varphi} g_{00}^{*}$ and $\bar{g}_{i j} \equiv g_{i j}^{*}$. If the coordinates have been chosen so that the Einstein metric $g_{\mu \nu}^{*}$ takes the Schwarzschild form (3.12), this is also the case for $\bar{g}_{\mu \nu}$ and one may thus use the integral expression (3.15). At first post-Newtonian order, all contributions coming from $g_{\mu \nu}^{*}$ reproduce the general relativistic result, ${ }^{14}$ and the only extra contribution is proportional to the scalar field, namely $\alpha \varphi=\alpha \varphi_{0}+\sqrt{G M a_{0}} \ln r / c^{2}+\mathcal{O}\left(1 / c^{4}\right)$ in the MOND regime. One thus gets straightforwardly

$$
\begin{aligned}
\Delta \theta & =\Delta \theta_{\mathrm{GR}}+4 \alpha \int_{r_{0}}^{\infty} \frac{\varphi(r)-\varphi\left(r_{0}\right)}{\left(1-r_{0}^{2} / r^{2}\right)^{3 / 2}} \frac{r_{0} d r}{r^{2}}+\mathcal{O}\left(\frac{1}{c^{4}}\right) \\
& =\Delta \theta_{\mathrm{GR}}+\frac{4 \sqrt{G M a_{0}}}{c^{2}} \int_{r_{0}}^{\infty} \frac{\ln \left(r / r_{0}\right)}{\left(1-r_{0}^{2} / r^{2}\right)^{3 / 2}} \frac{r_{0} d r}{r^{2}}+\mathcal{O}\left(\frac{1}{c^{4}}\right) \\
& =\Delta \theta_{\mathrm{GR}}+\frac{4 \sqrt{G M a_{0}}}{c^{2}}\left[\arcsin \left(\frac{r_{0}}{r}\right)-\frac{\left(r_{0} / r\right) \ln \left(r_{0} / r\right)}{\sqrt{1-r_{0}^{2} / r^{2}}}\right]_{r_{0}}^{\infty}+\mathcal{O}\left(\frac{1}{c^{4}}\right) \\
& =\Delta \theta_{\mathrm{GR}}+\frac{2 \pi \sqrt{G M a_{0}}}{c^{2}}+\mathcal{O}\left(\frac{1}{c^{4}}\right),
\end{aligned}
$$

which confirms that the light deflection angle is positive although photons are faster than in GR (with respect to the Einstein metric $g_{\mu \nu}^{*}$ ). This angle is exactly the one predicted by GR, when a spherical halo of dark matter is assumed to generate a Newtonian potential $\sqrt{G M a_{0}} \ln r$. Moreover, the first line makes it clear that the background value of $\varphi$ does not contribute to the integral. It must be stressed that the $\operatorname{sign}$ of $\varphi$ is not crucial in itself; what is important is that its derivative is positive. It is therefore possible to have a cosmological background such that $\alpha \varphi<0$ locally whereas the amount of light deflection remains the same. This remark will be of great importance in Sec. IVC, since we will show that an empirical fact strongly supports that gravitons should propagate at a speed greater (or equal) than the speed of photons (i.e., that $\alpha \varphi$ should be negative).

Although the above construction seems natural (apart from the existence of a preferred frame), let us however underline that it is actually quite ad hoc. Indeed, the relation (3.30) has been imposed by hand precisely to mimic GR's metric, but any other relation between $g_{00}^{*}$ and $g_{i j}^{*}$ would have predicted another light deflection angle. Even without considering the general case (3.28), where $A(\varphi)$ and $B(\varphi)$ are fully independent functions of the scalar field, it is instructive to analyze a disformal coupling slightly generalizing Eq. (3.31), namely

$$
\tilde{g}_{\mu \nu}=-e^{2 \alpha \varphi} U_{\mu} U_{\nu}+e^{-2 \kappa \alpha \varphi}\left(g_{\mu \nu}^{*}+U_{\mu} U_{\nu}\right),
$$

where $\kappa$ and the matter-scalar coupling constant $\alpha$ are dimensionless numbers to be constrained by experiments. The tightest experimental constraints come from solar-system tests, i.e., in the Newtonian regime of such theories, where one must have $\varphi=\varphi_{0}-\alpha G M / r c^{2}$. A nonvanishing background value $\varphi_{0}$ of the scalar field is necessary to respect the inequality $\alpha \varphi \geq 0$, imposed in Ref. [30] to avoid superluminal gravitons. Since $G M / R c^{2} \leq \frac{1}{2}$ for any body of mass $M$ and radius $R$, it suffices to impose $\varphi_{0} / \alpha \geq \frac{1}{2}$. (Let us however recall that superluminal gravitons are actually not a problem, as discussed in

14 Actually, there is a subtle modification of the Einstein metric $g_{\mu \nu}^{*}$ itself in TeVeS, because of the contribution of the energy-momentum tensor of the unit vector $U_{\mu}$; see Eq. (58) of [30] and Eq. (6.2) of [31]. However, this correction is chosen to be negligible in TeVeS. 
Sec. IIE above and in Ref. [103].) In this Newtonian regime, metric (3.33) reads thus $\tilde{g}_{00}=\exp \left(2 \alpha \varphi_{0}\right)\left[-1+2\left(1+\alpha^{2}\right) G M / r c^{2}\right]+\mathcal{O}\left(1 / c^{4}\right)$ and $\tilde{g}_{i j}=\exp \left(-2 \kappa \alpha \varphi_{0}\right) \delta_{i j}[1+2(1+$ $\left.\left.\kappa \alpha^{2}\right) G M / r c^{2}\right]+\mathcal{O}\left(1 / c^{4}\right)$, where the constant exponential factors may be eliminated by a redefinition of (physical) time and length units. The Eddington post-Newtonian parameter $\gamma^{\mathrm{PPN}}$ is thus given by

$$
\gamma^{\mathrm{PPN}}=\frac{1+\kappa \alpha^{2}}{1+\alpha^{2}}=1+\frac{(\kappa-1) \alpha^{2}}{1+\alpha^{2}} .
$$

The Cassini constraint [88] on this parameter imposes therefore $|\kappa-1| \alpha^{2} /\left(1+\alpha^{2}\right)<2 \times 10^{-5}$. In conclusion, experiment forces us to fine tune $\kappa \approx 1$, otherwise the matter-scalar coupling constant $\alpha$ needs to be small and we get back the serious problem pointed out in Sec. IIF above: The MOND logarithmic potential starts manifesting at too small distances, and one needs unnatural RAQUAL kinetic terms like the one of Fig. 3 to pass solar-system tests. [Note that $\kappa=1 \pm 0.1$ suffices for the Newton-MOND transition to be much smoother that in this Figure, allowing a Newtonian regime up to distances $\sim 100 \mathrm{AU}$ instead of 30 AU, but this remains nevertheless a quite fine-tuned behavior.] On the other hand, if $\kappa=1$ strictly, as chosen in Refs. [30, 31, 32, 33], there is no longer any post-Newtonian solar-system constraint on the matter-scalar coupling constant $\alpha$, and one may thus a priori choose $\alpha=1$ and a RAQUAL kinetic term of the natural form of Fig. 2. [The fact that $\kappa=1$ implies $\gamma^{\mathrm{PPN}}=1$ has been noticed in Ref. [30]. However, its author did not choose $\alpha=1$ (i.e., $k=4 \pi$ in the TeVeS notation) but a smaller value $\alpha \approx 5 \times 10^{-2}$ (i.e., $k \approx 0.03$ ). The reason was that he wished to neglect the energy-momentum tensor of the scalar field $\propto \alpha^{2}$, but it is actually negligible even when $\alpha=1$ since it is of second post-Newtonian order $\mathcal{O}\left(1 / c^{4}\right)$.]

This discussion illustrates that several experimental constraints have already been used to construct the model proposed in Refs. [30, 31, 32, 33]. Moreover, one cannot claim that it predicts the right light deflection, but rather that it has been tuned in order to do so. A vivid way to illustrate the fine tuning hidden in the choice (3.30)-(3.31) is to exhibit different metrics of the form (3.28), reproducing GR's light deflection in the Newtonian regime of the solar system but a different one in the MOND regime. For instance, if one writes this physical metric as ${ }^{15}$

$$
\tilde{g}_{\mu \nu}=-f_{0}(\varphi) U_{\mu} U_{\nu}+f_{r}(\varphi)\left(g_{\mu \nu}^{*}+U_{\mu} U_{\nu}\right),
$$

then the choice $f_{0}(\varphi)=e^{2 \varphi}$ and $f_{r}(\varphi)=e^{-2 \varphi /(1+\varepsilon \varphi)}$ reproduces the above model when $\varphi \ll 1 / \varepsilon$, but predicts a smaller light deflection when $\varphi>1 / \varepsilon$, notably when $\varphi \propto \ln r \rightarrow \infty$ in the MOND regime. On the contrary, the choice $f_{0}(\varphi)=e^{2 \varphi}$ and $f_{r}(\varphi)=e^{-2 \varphi(1+\varepsilon \varphi)}$ would also mimic the above model for $\varphi \ll 1 / \varepsilon$, but predict a much larger light deflection when $\varphi>1 / \varepsilon$, in the MOND regime. Therefore the specific choice (3.30)-(3.31), mimicking GR, may almost be considered as a fit of some experimental data. It is nevertheless rather natural, and the fact that it allows to reproduce GR's predictions in presence of dark matter is anyway a great achievement, since light deflection was a crucial difficulty of previous constructions.

Further analysis of such stratified theories however undermines this conclusion, and we will see that they cannot survive other phenomenological constraints, unless they are much more fine-tuned than they already are.

15 To simplify the discussion, we assume here that the asymptotic value $\varphi_{0}$ vanishes, or, stated differently, we merely denote here $\varphi-\varphi_{0}$ as $\varphi$. 


\section{DIFFICULTIES WITH STRATIFIED THEORIES}

\section{A. Stability issues}

One of the great advantages of the stratified TeVeS metric (3.28), over the disformally coupled scalar field (3.21), is the simplicity of the field equations within matter. However, as we have seen, the vector field needs to have a constant norm unless consistency problems occur in its field equation, and this has two serious drawbacks. First, this leads to a mixing of the vector and the spin-2 degrees of freedom and thereby to a nondiagonal system of differential equations. This means that the actual degrees of freedom have to be computed carefully (as done in [107] in the specific case $\tilde{g}_{\mu \nu}=g_{\mu \nu}^{*}$ ). Second, the fact that the vector field must be of constant norm spoils its stability. Indeed, as was shown in Ref. [34], the Hamiltonian of the vector field is not bounded by below, and the theory is unstable. The dynamics of the vector field derives from the action

$$
S=\int \mathcal{L} d^{4} x=-\frac{K c^{3}}{32 \pi G} \int \sqrt{-g^{*}} d^{4} x\left[g_{*}^{\mu \rho} g_{*}^{\nu \sigma} F_{\mu \nu} F_{\rho \sigma}-2 \lambda\left(g_{*}^{\mu \nu} U_{\mu} U_{\nu}+1\right)\right],
$$

where the stress $F_{\mu \nu}$ is given by $\partial_{\mu} U_{\nu}-\partial_{\nu} U_{\mu}, \mathcal{L}$ is the Lagrangian density, and $K$ is a positive dimensionless number. The Hamiltonian can be computed in the standard way, noticing that both $\lambda$ and $U_{0}$ must be treated as Lagrange multiplier since the action does not involve their time derivatives. The momenta read then

$$
\pi^{i}=-\frac{K c^{3}}{8 \pi G} F^{0 i}
$$

where latin indices run from 1 to 3 , and indices are raised using the inverse Einstein metric $g_{*}^{\mu \nu}$. In the limit of decoupling gravity $\left(g_{\mu \nu}^{*} \rightarrow \eta_{\mu \nu}\right)$, the Hamiltonian reads

$$
\begin{aligned}
H & =\int d^{3} x\left(\pi^{i} \partial_{0} U_{i}-\mathcal{L}\right) \\
& =\frac{K c^{3}}{8 \pi G} \int d^{3} x\left(\frac{F_{\mu \nu} F^{\mu \nu}}{4}-F^{0 i} \partial_{0} U_{i}\right),
\end{aligned}
$$

where we used that $U_{\mu} U_{*}^{\mu}=-1$ which derives from the equation $\delta S / \delta \lambda=0$. One then write $\partial_{0} U_{i}$ as $F_{0 i}+\partial_{i} U_{0}$ and integrates by parts the last term. The Hamiltonian, as a functional of the independent field configurations $U_{i}$ and $\pi^{i}$, then reads

$$
H=\int d^{3} x\left[\frac{4 \pi G}{K c^{3}} \boldsymbol{\pi}^{2}+\frac{K c^{3}}{4 \pi G}(\boldsymbol{\nabla} \times \mathbf{U})^{2}+\sqrt{1+\mathbf{U}^{2}} \nabla \boldsymbol{\pi}\right],
$$

where bold-faced symbol denotes three-vectors. The first two terms are just proportional to $E^{2}+B^{2}$ where $E$ and $B$ are the usual electric and magnetic fields associated to $U_{\mu}$. Contrary to the Maxwell case where the $\boldsymbol{\nabla} \boldsymbol{\pi} \propto \boldsymbol{\nabla} \mathbf{E}=0$ in vacuum, the last term does not vanish here (even in vacuum), and can take arbitrarily large and negative values. This can be made more explicit, for instance, by considering the special case where the vector field derives from a static potential $\phi$, i.e., $U_{i}=\partial_{i} \phi(x)$; see Ref. [34]. The Hamiltonian is thus not positive definite, and the theory is unstable.

We stressed in Sec. IID that a competing model (phase coupling gravitation) also reproduces the MOND dynamics. The theory is however unstable because of the presence of a 
negative potential. Recently, an improved version of this theory (called BSTV) was given in Ref. [33]. It involves, besides the two scalar fields, a dynamical unit vector field in order to reproduce the right amount of light deflection using a disformal metric of type Eq. (3.31).

This improved theory is however still unstable. Three different terms of the full Hamiltonian can take it to arbitrary large and negative values. To begin with, there is the instability driven by the vector field that we described above, if its kinetic term if of the Proca form (like in TeVeS). The dynamics of two scalar fields $q$ and $\varphi$ derives from the action ${ }^{16}$

$$
S=-\int \sqrt{-g^{*}} d^{4} x\left(\frac{1}{2}\left(\partial_{\mu} q\right)^{2}+\left[h(q) g_{*}^{\mu \nu}+(f(q)-h(q)) U_{*}^{\mu} U_{*}^{\nu}\right] \partial_{\mu} \varphi \partial_{\nu} \varphi+V(q)\right) .
$$

Because the author of Ref. [33] wrote a quadratic potential $V(q)=-A q^{2}$ with $A>0$, the field $q$ is tachyonic and thus unstable. Note that this potential is not crucial to obtain MOND dynamics, but is merely added to produce oscillations of the scalar field that may mimic the cosmic microwave background (CMB) predicted in cold dark matter theories. Curing the instability by changing the sign of $A$ may however spoil this interesting phenomenology. The computation of the full Hamiltonian reveals another instability. The momenta read $p_{q}=\partial_{0} q$ and $p_{\varphi}=2 h \partial_{0} \varphi-2\left(U_{\mu} \partial^{\mu} \varphi\right)(h-f) U_{0}$. This last equality can be inverted to find $\partial_{0} \varphi$ as a function of (notably) $p_{\varphi}$. Since the resulting Hamiltonian has a quite complicated expression, we only report its value on the particular field configuration such that $U_{i}=0$. Up to the contribution of the vector field, we have:

$$
H=\int d^{3} x\left(\frac{p_{q}^{2}+\left(\partial_{i} q\right)^{2}}{2}-A q^{2}+\frac{p_{\varphi}^{2}}{4[2 h(q)-f(q)]}+h(q)\left(\partial_{i} \varphi\right)^{2}\right) .
$$

Changing the sign of $A$ would not be sufficient to guarantee the stability of the theory. Indeed, the functions $h$ and $f$ must behave as $h(q)=q^{2}$ and $f(q)=q^{6}$ (at least in some regime) in order to obtain MOND dynamics. Therefore the term $p_{\varphi}^{2} / 4(2 h-f)$ can be made arbitrarily large and negative.

\section{B. Preferred-frame effects}

Ni's stratified theory [118] is a particular case of disformal coupling (3.31) where the Einstein metric is no longer dynamical, and assumed to be flat: $g_{\mu \nu}^{*}=\eta_{\mu \nu}$. Although it was built to reproduce the Schwarzschild solution at linear order, by imposing $e^{2 \alpha \varphi}=1-$ $2 G M / r c^{2}$, it is nevertheless known to be inconsistent with preferred-frame tests. Indeed, it predicts a post-Newtonian parameter $\alpha_{1} \approx-8$, whereas the present experimental constraint is $\left|\alpha_{1}\right|<10^{-4}[1,[2]$. One may thus wonder if the more general models of Sec. IIID present the same difficulty.

Their experimental constraints have been discussed in Ref. [32], as well in Ref. [121] in a more general context. In the case where the preferred frame defined by $U_{\mu}$ is assumed to be the CMB rest frame (as is usually done when analyzing the weak-field predictions of vector-tensor theories), Ref. [32] has proven that the post-Newtonian parameter $\alpha_{1} \approx-16 \alpha^{2}$ (where $\alpha$ without any index denotes as before the matter-scalar coupling constant, related

${ }^{16}$ Beware that Ref. 33] uses the opposite (mostly -) signature. We have translated its action in our mostly + convention. 
to the parameter $\eta$ of Ref. [32] by $\eta=4 \alpha^{2}$ ). The other PPN parameters characterizing preferred-frame effects vanish identically, like in general relativity $\left(\alpha_{2}=\alpha_{3}=0\right)$. The present experimental bound on $\alpha_{1}$ would thus impose $\alpha^{2}<6 \times 10^{-6}$, even tighter than the light-deflection constraint $\alpha^{2}<10^{-5}$ on conformally-coupled scalar-tensor theories. The fine-tuning problem discussed in Sec. IIF above would thus be even more serious in the present disformal framework.

However, the vector field $U_{\mu}$ is dynamical, and Refs. [30, 31] claim that its field equation drives it to be parallel to the matter proper time, i.e., to read $U_{\mu}=( \pm 1,0,0,0)$ in the matter rest frame. In such a case, there would be no preferred-frame effect due to the motion at $370 \mathrm{~km} . \mathrm{s}^{-1}$ of the solar-system with respect to the CMB, but there could still exist observable effects due to the planets' velocities around the Sun. The Earth's velocity at about $30 \mathrm{~km} \cdot \mathrm{s}^{-1}$ would a priori induce effects constrained by the tracking of artificial satellites [122], and although the bound on $\alpha^{2}$ might be increased by a factor 10, the finetuning described in Sec. IIF would still be necessary. It remains possible that the vector field is also driven to be parallel to the Earth's proper time in its vicinity, but there would always exist a transition region between the barycentric and geocentric frames in which test particle should undergo observable effects due to the variation of $U_{\mu}$.

Anyway, the derivation proposed in [30, 31] actually only proves that $U_{\mu}=( \pm 1,0,0,0)$ is $a$ solution, but not the solution. In other words, it just tells us that a situation where matter is at rest with respect to the preferred frame is possible. This obviously does not prove that the model is consistent with high-precision tests of local Lorentz invariance of gravity. There remains to perform a careful analysis of the specific models proposed in [30, 31, 33] in order to draw definite conclusions about this problem of preferred-frame effects. We will not discuss it any longer in the present paper, because stratified theories anyway present several other serious difficulties.

\section{Cerenkov radiation and high-energy cosmic rays}

Throughout this paper, we insisted on the fact that superluminal fields do not necessarily threaten causality, provided they propagate along cones in spacetime, i.e., if their equations of motion remain hyperbolic. We even announced in Sec. IIF that experiment strongly suggests that no field should propagate slower than light. Indeed highly relativistic matter coupled to a subluminal field emits Cerenkov radiation and thereby loses energy. In particular, the various gravitational fields one may consider should not propagate slower than light, otherwise ultra high energy cosmic rays would emit "gravi-Cerenkov" radiation (following the term used in [35, 36]), and those that we detect should thus have been produced near the Earth. However, no source has been identified within a range of the order of the kiloparsec, and this yields a tight bound on how much photons (and hence relativistic matter particles) are allowed to travel faster than gravity.

Cerenkov radiation occurs in electrodynamics when charged particles propagate faster than light in a medium with refractive index $n>1$. The emission of a real photon through the vertex of QED is kinematically allowed in that case because photons do not follow the usual dispersion law $p_{\mu} p^{\mu}=0$, but satisfy the law $p_{0}=n|\mathbf{p}|$ in a refractive medium and carry thus more energy than momentum if $n>1$; see [35, 36].

If light propagates faster than gravitational waves, as is the case in the above stratified theory with $\alpha \varphi>0$, a similar phenomenon occurs: (ultra relativistic) matter particles propagating faster than gravity emit gravitational waves. The same reasoning as above 
shows that this tree process is kinematically allowed. We may however stress a subtlety here. Since spacetime is endowed with two different metrics of Lorentzian signature, one can define two different sets of locally inertial coordinates (for which one of the two metrics $g_{\mu \nu}^{*}$ or $\tilde{g}_{\mu \nu}$, but not both, is written in its canonical form). There exists therefore two (equivalent) formulations of conservation laws. Gravitational waves follow the null geodesic of the gravitational metric ${ }^{17}$ and satisfy thus the equation $g_{\mu \nu}^{*} p^{\mu} p^{\nu}=0$, where $p^{\mu}$ denotes their four-momentum. Their dispersion relation in the matter (Jordan) frame reads thus $\tilde{g}_{\mu \nu} p^{\mu} p^{\nu}=B\left(U_{\mu} p^{\mu}\right)^{2}$, and takes the form

$$
p^{0}=|\mathbf{p}| / \sqrt{1-2 \sinh (2 \alpha \varphi)}
$$

in coordinates such that $\tilde{g}_{\mu \nu}=\eta_{\mu \nu}$ and simultaneously $U_{\mu}=( \pm 1,0,0,0)$ locally (which can always be imposed by a local Lorentz transformation keeping $\left.\tilde{g}_{\mu \nu}=\eta_{\mu \nu}\right)$. Using $\alpha \varphi \ll 1$, we get $p^{0}=|\mathbf{p}|(1+2 \alpha \varphi)$. In other words, everything behaves as if gravitons were travelling within a refractive medium of index $n \approx 1+2 \alpha \varphi$. The emission of real gravitational waves by ultra relativistic matter particles is thus allowed once $\alpha \varphi>0$. The authors of Ref. [35] calculated the rate of energy loss by this process and found:

$$
\frac{d E}{d t} \approx \frac{G p^{4}(n-1)^{2}}{\hbar^{2} c_{\text {light }}}
$$

for a particle of momentum $p$. Here we approximate pure numbers by 1 , which is correct in view of the exact results derived in 35]. [This result is actually obtained for a scalar particle of matter; the case of a proton is more involved but leads to similar formulae; see Ref. [36]]. This equation is easily integrated for an ultra-relativistic particle $E \approx p c_{\text {light }}$. Assuming that the initial value of the momentum $p_{i}$ is much larger than the final one $p_{f}$ and that $n$ is approximatively constant along the particle's path, one finds that it can travel only over a distance of order

$$
L \sim \frac{\hbar^{2} c_{\text {light }}^{3}}{G p_{f}^{3}(n-1)^{2}},
$$

whose value reads

$$
L_{\max } \sim \frac{2 \times 10^{-11}}{(n-1)^{2}} \mathrm{~m}
$$

for $p_{f} \sim 10^{20} \mathrm{eV}$. Therefore, cosmic rays detected on Earth with such a high momenta as $10^{20} \mathrm{eV}$ cannot have been produced further than $L_{\max }$. Assuming that these cosmic rays have at least a galactic origin, we find $L_{\max } \gtrsim 10^{21} \mathrm{~m}$ and therefore $n-1 \lesssim 10^{-16}$. Note of course that gravi-Cerenkov radiation can only be emitted by matter particles propagating at a speed greater than $c_{\text {gravitons }}=c_{\text {light }} / n$, where $n-1 \lesssim 10^{-16}$. Therefore only ultrarelativistic particles with a Lorentz-Fitzgerald factor of order $\gamma \geq 1 / \sqrt{2(n-1)} \sim 10^{8}$ can emit gravitational waves in this way. Assuming that the observed high-energy cosmic rays with momenta $p \sim 10^{20} \mathrm{eV}$ are protons, we find that their $\gamma$ reads $10^{11}$, so that our analysis is self-consistent.

17 Beware that the spectrum of gravitational waves may be affected by the presence of the vector field. However, when the kinetic term of the vector field is of the Proca type, there is no such modification; see Ref. [123]. 
Since the gravitational field produced by the Milky Way is approximatively MONDian in the vicinity of the Sun, we have $n-1 \approx 2 \alpha \varphi \approx 2 \sqrt{G M_{\text {Milky Way }} a_{0}} \ln (r / \ell) / c^{2}$, where $\ell$ is a fixed length scale and where, numerically, $\sqrt{G M_{\text {Milky Way }} a_{0}} / c^{2} \sim 10^{-7}$. If one insists on the fact that $\alpha \varphi$ should be positive (so that gravity propagates slower than photons), the only way inequality $n-1 \lesssim 10^{-16}$ could be satisfied would be to fine tune the length scale $\ell$ to the galactocentric distance of the Sun up to nine decimals! In other words, such a theory predicts that high energy cosmic rays should not be seen unless they are produced very close to the Earth, or if the Sun were very specifically located within our galaxy. This is clearly unacceptable, and $\alpha \varphi$ would anyway become negative for slightly smaller galactocentric distances. In other words, gravity must propagate faster than light at least in some regions.

The only reasonable way to avoid the above fine tuning problem is simply to have $\alpha \varphi<$ 0 . In such a case, light propagates slower than gravitons and there is no gravi-Cerenkov radiation at all. ${ }^{18}$ Let us emphasize that it is however in great disagreement with the whole spirit of the TeVeS theory. Indeed the vector field was precisely introduced to produce the right amount of light deflection while avoiding any superluminal field. Actually, the above discussion shows that subluminal fields coupled to the matter sector are generically ruled out by the existence of ultra high-energy cosmic rays. Therefore MOND-like stratified theories reproducing the right light deflection must either be based on the disformal relation (3.21) (where the vector field assumes the form $U_{\mu}=\partial_{\mu} \varphi$ ) with $B<0$, or on the disformal metric Eq. (3.31) with $\alpha \varphi<0$. In conclusion, light should in any case travel slower than gravitons. Let us recall that it does not imply a violation of causality, provided all field equations remain hyperbolic.

\section{Binary-pulsar constraints}

The fourth and probably most serious difficulty of stratified theories is their consistency with binary-pulsar observations. Indeed, even if the choice $\kappa=1$, in Eq. (3.34), releases the constraint on $\alpha$ arising from precise measurement of the Eddington parameter $\gamma^{\mathrm{PPN}}$ in the solar system, binary-pulsar data a priori impose a tight constraint on it too. The literature on binary-pulsar tests has studied wide classes of alternative theories of gravity $[1,89,191,92,124,125,126]$ but not the disformal coupling (3.28). A specific analysis should thus be performed, but it goes beyond the scope of the present article. Let us just mention here that a strong matter-scalar coupling constant $\alpha \approx 1$ means that the spin- $2\left(g_{\mu \nu}^{*}-\eta_{\mu \nu}\right)$ and spin- $0\left(\varphi-\varphi_{0}\right)$ fields are of the same order of magnitude in the vicinity of massive bodies, i.e., in the Newtonian regime of the model. Near a binary system, these fields cannot be static because they are driven by the orbital motion. This causes the emission of gravitational and scalar waves (and even waves of the vector field $U_{\mu}$, in the present TeVeS model), which extract energy from the system and make the orbit shrink. Because of the specific form of the kinetic terms (i.e., of the helicities of the degrees of freedom), the energy loss via spin-2 waves start at the quadrupolar order $\mathcal{O}\left(1 / c^{5}\right)$, but it is of the much larger order $\mathcal{O}\left(1 / c^{3}\right)$

18 Note that one may also consider Cerenkov radiation made of waves of the vector field. Indeed, depending on the sign of the parameters, such "Einstein-aether" waves [123] may propagate slower than light. In any case, however, it would be possible to require that $\alpha \varphi$ is negative enough so that light (and hence matter) propagates slowly enough, to ensure that matter particles do not emit such Cerenkov radiation at all. 
for the dipolar spin-0 waves [monopolar scalar waves also exist but cause an energy loss of order $\mathcal{O}\left(1 / c^{5}\right)$ instead of $\mathcal{O}(1 / c)$ if the two orbiting bodies are at equilibrium]. The orbit should thus shrink much more quickly in the present model than in GR, and this is known to be inconsistent with several binary-pulsar observations [89, 91, 92, 124, 125, 126, 127, 128]. Since this reasoning only depends on the local dynamics of the scalar field, its subtle MONDlike behavior at larger distances should not change the conclusion. The scalar waves will obviously behave in a non-standard way at distances $r \gtrsim \sqrt{G M / a_{0}}$, but they have anyway extracted too much energy from the binary system in the near zone. Note also that the peculiar matter-scalar coupling (3.30)-(3.31) should not change either the above conclusion. It tells us that $\varphi$ does not feel the matter current and pressure $T_{i j}^{* \text { matter }}$ as in standard scalartensor theories, but the dominant source remains $\alpha T_{00}^{* \text { matter }} \propto \alpha M c^{2}$ and generates a scalar field $\varphi-\varphi_{0}=\mathcal{O}\left(\alpha G M / r c^{2}\right)$. Therefore, we expect present binary-pulsar data to impose $\alpha^{2}<4 \times 10^{-4}$, as in Brans-Dicke theory [124, 125, 126]. Although this is less constraining than the $10^{-5}$ bound imposed by solar system [88] on conformally-coupled scalar fields (2.12)-(2.13), this binary-pulsar constraint on the matter-scalar coupling constant is thus $a$ priori also valid for the disformally coupled ones (3.21)-(3.28) ${ }^{19}$

Note that this constraint is one order of magnitude smaller than the value $\alpha^{2} \approx 2 \times 10^{-3}$ chosen in TeVeS [31] (where $\alpha^{2}=k / 4 \pi$ in Bekenstein's notation). This reference needed to choose $\alpha^{2}>1.4 \times 10^{-3}$ in order to predict negligible MONDian effects at distances smaller than Saturn's orbit, assuming a natural RAQUAL kinetic term like the one displayed in Fig. 2 above. Since this is inconsistent with binary-pulsar tests, we recover again the problem discussed in Sec. IIF: $\alpha$ needs to be small and we need an unnatural kinetic term $f(s)$. In particular, the acceleration cannot be purely Newtonian beyond $r \sim 150$ AU (if $\alpha^{2}=4 \times 10^{-4}$, and an approximately constant extra contribution $a_{0}$ must exist between this distance and $\sqrt{G M / a_{0}} \approx 7000 \mathrm{AU}$, where it becomes of the $1 / r$ MOND form (see Fig. 3). Although this is still allowed experimentally, this behavior seems anyway too fine-tuned.

\section{E. Discussion}

As Secs. IIID and IV] above underline, the TeVeS model [30, 31, 33] is certainly the best field theory reproducing the MOND dynamics in the literature, but it has still some drawbacks. One of them is probably unavoidable: It is fine tuned to obtain the wanted phenomenology. A second one seems generic but hopefully curable in some specific models: It presents some instabilities. Other theoretical problems should be solved, and have already received some answers: It involves discontinuities, too many different fields, and the various arbitrary functions it contains look quite complicated. But the most serious difficulties seem to be experimental, as discussed in the present section. In particular, it very probably does not pass binary-pulsar tests in its present form (or needs to be even more fine-tuned in a quite unnatural way). We will also mention in Sec. VI other generic problems that any MOND-like field theory should address. Therefore, in spite of the great achievements of Refs. [30, 31, 33], it remains interesting to look for other theoretical frameworks able to

19 This discussion is reminiscent of a particular tensor-bi-scalar theory constructed in 89] as a contrasting alternative to GR. It depends on two free parameters characterizing how the two scalar fields are coupled to matter. Even for strong couplings, the model was fine tuned to pass anyway solar-system tests. The combination of various binary-pulsar tests however severely constrains both of the parameters [89, 126]. 
reproduce the MOND dynamics in a consistent way. We will examine a rather new one in the following section, although it is still in the class of RAQUAL and disformal models. Its original feature is that the theory will be particularly simple in vacuum (outside matter sources), namely pure general relativity in a first model, and pure Brans-Dicke theory in a second one. However, we will see that the consistency of the field equations within matter exhibits subtle but crucial difficulties.

\section{NONMINIMAL METRIC COUPLINGS}

\section{A. Changing the matter-spin 2 coupling}

In the present section, we adopt a mixing of the modified-inertia and modified-gravity viewpoints discussed in Sec. II A above. Like in the modified-inertia framework, the dynamics of gravity in vacuum will be defined by the Einstein-Hilbert action (2.1). On the other hand, like in modified gravity, the matter action will be assumed to depend only on the matter fields and their first derivatives, or, in the case of test particles, on their positions and velocities only. Moreover, in order to preserve the weak equivalence principle, we will assume that all matter fields are coupled to a single second rank symmetric tensor $\tilde{g}_{\mu \nu}$. In other words, the action of the theory reads

$$
S=\frac{c^{4}}{16 \pi G} \int \frac{d^{4} x}{c} \sqrt{-g_{*}} R^{*}+S_{\text {matter }}\left[\psi ; \tilde{g}_{\mu \nu}\right]
$$

where as before $\psi$ denotes globally any matter field. Therefore, we do not seem to have changed anything with respect to GR, and this looks like a model in which neither inertia nor gravity are modified! However, the crucial difference with GR is that we will assume the physical metric $\tilde{g}_{\mu \nu}$ to depend not only on the Einstein (spin-2) metric $g_{\mu \nu}^{*}$, but also on the curvature tensors one may construct from it and their covariant derivatives. Several definitions of $\tilde{g}_{\mu \nu}$ yield the right MOND phenomenology at leading order, and differ only at the post-Newtonian level, i.e., for relativistic corrections of order $\mathcal{O}\left(1 / c^{2}\right)$ with respect to the Newtonian and MOND accelerations. Let us choose a particular example:

$$
\tilde{g}_{\mu \nu} \equiv g_{\mu \nu}^{*}+\frac{\sqrt{a_{0} / 3}}{4 c} \frac{\left(\partial_{\lambda} \mathrm{GB}\right)^{2} h(X) g_{\mu \nu}^{*}+2 \partial_{\mu} \mathrm{GB} \partial_{\nu} \mathrm{GB}}{(\square * \mathrm{~GB} / 10)^{7 / 4}},
$$

where GB $\equiv R_{\mu \nu \rho \sigma}^{* 2}-4 R_{\mu \nu}^{* 2}+R_{*}^{2}$ is the Gauss-Bonnet topological invariant (which may be replaced by $R_{\mu \nu \rho \sigma}^{* 2}$ or the square of the Weyl tensor $C_{\mu \nu \rho \sigma}^{* 2}$ without changing the results at first order in $\sqrt{a_{0}}$ ), where $\square^{*}$ denotes the d'Alembertian operator (covariant with respect to the Einstein metric $g_{\mu \nu}^{*}$ ), and where

$$
X \equiv \frac{1}{\ell_{0}} \sqrt{\frac{30 \mathrm{~GB}}{\square * \mathrm{~GB}}} .
$$

The fixed length scale $\ell_{0}$ is introduced to make this quantity dimensionless. The numerical factors entering Eqs. (5.2)-(5.3) have been chosen to simplify the expression of the function $h(X)$, which will be specified below. Note that $\tilde{g}_{\mu \nu}$ is indeed a second-rank symmetric tensor, since it is constructed only from tensors, their covariant derivatives and their contractions. The matter action $S_{\text {matter }}\left[\psi ; \tilde{g}_{\mu \nu}\right]$ thereby satisfies exactly Lorentz invariance. Moreover, this 
is still a local action since the physical metric (5.2) involves a finite number of derivatives of $g_{\mu \nu}^{*}$. It should be noted that Eq. (5.2) does not make sense in flat spacetime, because it would involve ratios of vanishing expressions, but our Universe is never strictly flat since massive bodies do exist.

Since in Eq. (5.1), the physical metric $\tilde{g}_{\mu \nu}$ only enters the matter action, the curvature tensors it involves do not contribute to the dynamics of gravity in vacuum. This implies notably that the Birkhoff theorem is satisfied, like in GR. The metric generated by a spherically symmetric distribution of matter is thus of the Schwarzschild type, i.e., reads in Schwarzschild coordinates

$$
g_{\mu \nu}^{*} d x^{\mu} d x^{\nu}=-\left(1-\frac{2 G M}{r c^{2}}\right) c^{2} d t^{2}+\left(1-\frac{2 G M}{r c^{2}}\right)^{-1} d r^{2}+r^{2}\left(d \theta^{2}+\sin ^{2} \theta d \phi^{2}\right),
$$

where $M$ is a constant, very close to the one that GR would have defined (but slightly modified because of the nonminimal coupling of matter to curvature). It is then straightforward to compute the gravitational potential $V=-\frac{1}{2}\left(1+\tilde{g}_{00}\right) c^{2}$ felt by a test particle slowly moving in such a spacetime. The Schwarzschild solution (5.4) implies

$$
\begin{aligned}
\mathrm{GB} & =R_{\mu \nu \rho \sigma}^{* 2}=C_{\mu \nu \rho \sigma}^{* 2}=48\left(\frac{G M}{r^{3} c^{2}}\right)^{2}, \\
\square^{*} \mathrm{~GB} & =1440\left(\frac{G M}{r^{4} c^{2}}\right)^{2}\left(1-\frac{12}{5} \frac{G M}{r c^{2}}\right),
\end{aligned}
$$

so that the time-time component of the physical metric reads

$$
\tilde{g}_{00}=-1+\frac{2 G M}{r c^{2}}-2 \frac{\sqrt{G M a_{0}}}{c^{2}} h\left(\frac{r}{\ell_{0}}\right)+\mathcal{O}\left(\frac{1}{c^{4}}\right) .
$$

One thus recovers the MOND potential if one chooses $h(X)=\ln X$, and the length scale $\ell_{0}$ is then arbitrary since it does not enter any observable prediction. However, we know that the MOND potential should not be felt at small distances, because it would be inconsistent with solar-system tests of Kepler's third law [109]. One should thus choose a more finetuned function $h(X)$, such that its derivative gives a $1 / r$ force at large distances $r \gg \ell_{0}$ but a vanishing one when $r \ll \ell_{0}$. One may choose for instance $h^{\prime}(X)=X /(1+X)^{2}$, i.e., after integration,

$$
h(X)=(1+X)^{-1}+\ln (1+X) .
$$

Now the constant $\ell_{0}$ does enter the physical predictions, and in order to obtain the correct phenomenology, it should be chosen much larger than the solar-system size but small with respect to the radius of any galaxy. This underlines that the present model is also quite fine tuned, in a similar way as the RAQUAL models discussed in Secs. IIF and IID above. They needed specific aquadratic kinetic terms for the scalar field, and the transition between the Newtonian and MOND regimes then occurred at a radius $\sim \alpha^{2} \sqrt{G M / a_{0}}$, generically much too small to be consistent with solar-system tests. Here the transition scale $\ell_{0}$ is fixed by hand in Eq. (5.3), which may be considered as a bonus or a drawback. Note anyway that it would be easy to impose $\ell_{0}=\sqrt{G M / a_{0}}$, for instance, merely by using again Eqs. (5.5) and (5.6) to define this quantity and replace it in (5.3). However, the corresponding definition of $X \approx\left(\square^{*} \mathrm{~GB}\right)^{5 / 4} \sqrt{a_{0} \mathrm{~GB}} /\left(\partial_{\lambda} \mathrm{GB}\right)^{2} c$ would be even more unnatural! 
The light deflection can also be derived straightforwardly from the physical metric (5.2) expanded at first order in $1 / c^{2}$. Since photons do not feel any global factor of the metric, we may actually discard the term proportional to $\sqrt{a_{0}} g_{\mu \nu}^{*}$ in Eq. (5.2), modulo higher corrections $\mathcal{O}\left(a_{0} / c^{4}\right)$. Let us denote as $\bar{g}_{\mu \nu}$ the resulting metric, which suffices to derive the light path. The only remaining modification with respect to general relativity is the final term of Eq. (5.2), proportional to $\partial_{\mu} \mathrm{GB} \partial_{\nu} \mathrm{GB}$. In the case of the Schwarzschild solution (5.4), this term only contributes to the radial-radial component of the metric

$$
\bar{g}_{r r}=1+\frac{2 G M}{r c^{2}}+\frac{4 \sqrt{G M a_{0}}}{c^{2}}+\mathcal{O}\left(\frac{1}{c^{4}}\right) .
$$

We thus recover the disformal metric constructed in Sec. IIIC above, and the corresponding light deflection, Eq. (3.24), reproduces exactly (by construction) the result predicted by GR in presence of dark matter. Note that the extra (constant) deflection angle $2 \pi \sqrt{G M a_{0}} / c^{2}$ is also predicted in the solar system. It would be possible to suppress it by introducing another fine-tuned function $b(X)$ multiplying the $\partial_{\mu} \mathrm{GB} \partial_{\nu} \mathrm{GB}$ term in Eq. (5.2). However, this is not necessary, since this extra deflection angle is $\approx 2 \times 10^{-6} \operatorname{arcsec}$ for $M=M_{\odot}$, i.e., negligible with respect to present and foreseen experimental accuracy.

Therefore, the above model reproduces both the MOND dynamics and the correct light deflection in a particularly simple way. It does not introduce any superfluous degree of freedom in the vacuum, outside matter sources: It has the same spectrum as GR, namely a massless spin-2 field carrying positive energy, and does not contain any ghost nor tachyon. Moreover, it satisfies general covariance, Lorentz invariance (no preferred frame), locality, and the weak equivalence principle. Binary-pulsar tests are also obviously passed, since the metric $g_{\mu \nu}^{*}$ generated by a binary system takes exactly the same form as in GR. The only difference is that the masses slightly differ from those GR would have predicted for a given amount of matter. However, since these masses are not directly measured, but determined from the combination of various orbital observables, the analysis of binary-pulsar data is actually strictly the same as in GR.

It should also be underlined that nonminimal couplings to curvature, such as those introduced in (5.2), do appear in scalar-tensor theories and in GR itself when describing the motion of extended bodies [92, 129, 130]. Indeed, when tidal effects can be neglected (i.e., up to order $1 / c^{9}$ included, in GR), compact bodies are characterized only by their mass besides their position and velocity, and their action can thus be written $S_{\mathrm{pp}}=-\int m c \sqrt{-g_{\mu \nu}^{*} d x^{\mu} d x^{\nu}}$. As soon as tidal deformations start influencing their motion (from order $1 / c^{10}$ in GR), they must be described by more "form factors" characterizing how they are coupled to derivatives of the metric. Phenomenological couplings to $R_{\mu \nu \rho \sigma}^{* 2}$ and higher derivatives are thus expected to occur even within GR. Therefore, those entering Eq. (5.2) are rather natural, and define a consistent dynamics for massive point particles.

However, the above model also presents several difficulties. First of all, it has been finetuned to yield the right phenomenology, but other functions of the curvature tensors would obviously give a fully different physics. For instance, it suffices to change the numerical coefficient of the disformal contribution $\partial_{\mu} \mathrm{GB} \partial_{\nu} \mathrm{GB}$ in Eq. (5.2) to change the prediction for light bending by any amount. Therefore, this model is unpredictive, and just manages to fit some experimental data. Actually, this is probably one of its main interests: It illustrates, in a very simple framework, all the hypotheses which are also needed in other models of the literature. As discussed at the end of Sec. IIID above, the best present MOND-like field theory [30, 31, 33] could also predict a fully different light bending than GR if one had 
chosen different functions of the scalar field in Eq. (3.30).

A related problem is that there exist many different realizations of the physical metric $\tilde{g}_{\mu \nu}$ yielding the same phenomenology at lowest order. Indeed, Eq. (5.2) is actually a mere rewriting of the needed metric (5.7)-(5.9) in a covariant way. Any other combination of the Riemann curvature tensor and its covariant derivatives, reducing to the same metric at order $\mathcal{O}\left(1 / c^{2}\right)$, is thus equally valid. For instance, the crucial factor $\left(2 \sqrt{G M a_{0}} / c^{2}\right) g_{\mu \nu}^{*}$ may equivalently be written in terms of $\left(\square^{*} \mathrm{~GB}\right)^{-3 / 4} R_{\mu \rho \sigma \tau}^{*} R_{\nu}^{* \rho \sigma \tau}$. Any other nonvanishing contraction, like $\left(\nabla_{\mu}^{*} R_{\nu \rho \sigma \tau}^{*}\right)^{2}$ or $R_{*}^{\mu \nu}{ }_{0 \sigma} R_{* \kappa \lambda}^{\rho \sigma} R_{* \mu \nu}^{\kappa \lambda}$, may also be used to build the appropriate MOND potential. [On the other hand, neither the Ricci tensor $R_{\mu \nu}^{*}$, the scalar curvature $R^{*}$, nor any of their covariant derivatives, can be used to construct the physical metric $\tilde{g}_{\mu \nu}$, since they vanish in vacuum and thereby cannot be felt by a test particle far from the gravitational source.] The physical metric (5.2) that we chose above is convenient to write because it involves very few explicit indices, but it has no special property which distinguishes it from other possible choices. This freedom actually teaches us another important lesson: There is no reason, for a model reproducing the right phenomenology at lowest order, to remain valid at higher orders. In the present case, there is an infinity of ways to complete it at higher post-Newtonian orders, by adding arbitrary combinations of the curvature tensor and its covariant derivatives. In the case of the TeVeS model [30, 31, 33], there is no reason either to trust its higher post-Newtonian predictions.

Another related problem is that the variable $X$ defined in Eq. (5.3) may become imaginary in particular backgrounds where $\square^{*} \mathrm{~GB}<0$. One may of course take its absolute value in order to always define $X$ consistently, but it would anyway diverge on the hypersurfaces where $\square^{*} \mathrm{~GB}=0$. The full metric (5.2) is actually ill-defined on such hypersurfaces, since our "correcting terms" $\mathcal{O}\left(\sqrt{a_{0}}\right)$ may blow up. This illustrates once again that the specific choice (5.2) is not justified by any symmetry principle nor any fundamental theory. This is just a possible phenomenological realization of the MOND dynamics at lowest order, but not a complete theory remaining consistent in all physical situations. It is always possible to refine the model to avoid such pathological situations, as we did in Sec. IIF to avoid the singularity of RAQUAL models on the hypersurfaces where $s \equiv g_{*}^{\mu \nu} \partial_{\mu} \varphi \partial_{\mu} \varphi$ vanishes. However, such refinements do not make the models more fundamental, and there is still no reason to believe their predictions in situations very different from clustered matter (i.e., solar system and galaxies).

But the most serious difficulty of the nonminimally-coupled model (5.1)-(5.2) is its consistency within matter. First of all, the physical metric $\tilde{g}_{\mu \nu}$ should always remain hyperbolic, in order to define a well-posed Cauchy problem for matter fields. This is clearly the case if the correcting terms $\mathcal{O}\left(\sqrt{a_{0}}\right)$ entering (5.2) remain small with respect to the flat background metric $\eta_{\mu \nu}=\operatorname{diag}(-1,1,1,1)$. In more general situations where they become of order 1 , higher-order corrections are anyway expected to grow too, and the above phenomenological model just stops being predictive. Once again, the lowest-order MOND dynamics does not suffice to fully specify the physics in all regimes. The deadly problem arises in the field equations for gravity within matter. Indeed, since higher derivatives of $g_{\mu \nu}^{*}$ enter the matter action, the full Hamiltonian is not bounded by below, because of Ostrogradski's theorem discussed in Sec. [IC above. Therefore, this model is unstable, in the same way as the one proposed in Ref. [72], that we mentioned in Sec. [IC. Although one cannot identify any ghost degree of freedom around a flat empty background, the model [72] did contain negative-energy modes around curved backgrounds, and the present one (5.1)-(5.2) involves negative-energy modes within matter. This is illustrated in terms of Feynman diagrams in 
(a)

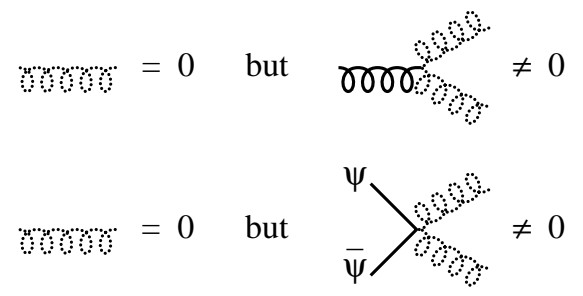

FIG. 6: Diagrammatic illustration of the negative-energy modes spoiling the stability of (a) the model proposed in [72], and (b) the present nonminimal metric coupling (5.1)-(5.2). As before, curly lines represent spin-2 degrees of freedom, namely the usual massless and positive-energy graviton in plain line, and the massive ghost in dotted lines. Contrary to Fig 5 , straight lines represent here matter fields $\psi$ instead of the scalar field $\varphi$. In both cases (a) and (b), no ghost degree of freedom can be identified around a flat or empty background. However, a ghost mode does exist in curved backgrounds in case (a), and within matter in case (b). They use the background gravitons or matter fields as "catalyzers" in order to propagate and develop instabilities.

Fig. 6.

As a simple toy model of such unstable theories, one may consider for instance the Lagrangian $\mathcal{L}=-\left(\partial_{\mu} \psi\right)^{2}\left[1+\lambda\left(\partial_{\nu} \varphi\right)^{2}\right]$ in flat spacetime, where $\lambda$ is a nonzero constant. The scalar $\psi$ is an analogue of the matter fields in our present model (5.1)-(5.2), and of the usual massless graviton in the model [72]. One cannot identify any kinetic term for the second scalar $\varphi$ around a background $\psi=$ const., but it is obvious that it defines a ghost degree of freedom in backgrounds where $\lambda\left(\partial_{\mu} \psi\right)^{2}<0$. More generally, one can easily compute the Hamiltonian $\mathcal{H}=\left[\dot{\psi}^{2}+\left(\partial_{i} \psi\right)^{2}\right]\left[1+\lambda\left(\partial_{j} \varphi\right)^{2}\right]+\lambda \dot{\varphi}^{2}\left[\left(\partial_{i} \psi\right)^{2}-3 \dot{\psi}^{2}\right]$, and prove that there always exist initial conditions such that $\mathcal{H} \rightarrow-\infty$. If $\lambda>0$, it suffices for instance to choose $\partial_{i} \psi=\partial_{i} \varphi=0, \dot{\varphi}^{2}=1 / \lambda, \dot{\psi}^{2} \rightarrow \infty$, and if $\lambda<0$, to choose $\partial_{i} \varphi=0$, $\dot{\psi}=0,\left(\partial_{i} \psi\right)^{2} \dot{\varphi}^{2} \rightarrow \infty$. Both cases can be expressed the same way in terms of the conjugate momenta $\pi_{\psi} \equiv \partial \mathcal{L} / \partial \dot{\psi}$ and $\pi_{\varphi} \equiv \partial \mathcal{L} / \partial \dot{\varphi}$ : Whatever the sign of $\lambda \neq 0$, one gets $\mathcal{H} \rightarrow-\infty$ for $\partial_{i} \varphi=0, \pi_{\psi}=0, \partial_{i} \psi \rightarrow 0$ and $\pi_{\varphi}^{2} \rightarrow \infty$.

In the present model (5.1)-(5.2), the simplest way to understand the existence of negativeenergy modes, confined within matter, is to consider a homogeneous density (either filling the Universe, as in standard cosmological models, or in the interior of a massive body). Then the matter action $S_{\text {matter }}\left[\psi ; \tilde{g}_{\mu \nu}\right]$ actually defines a gravity theory of the form $f\left(R_{\mu \nu \rho \sigma}^{*}, R_{\mu \nu}^{*}, \nabla_{\lambda}^{*} R_{\mu \nu \rho \sigma}^{*}, \ldots\right)$, which is known to contain a massive spin-2 ghost degree of freedom [68, 70, 71], as recalled in Sec. IIC. Of course, one may invoke again higher-order corrections to the phenomenological model (5.2), which may be able to stabilize it. However, it remains that it is unstable without considering such higher-order corrections, and therefore that its lowest-order truncation (5.2) cannot describe consistently massive bodies. At the classical level, one should analyze carefully this instability to determine its characteristic timescale. Naively, one could expect it to be related to the MOND acceleration scale $a_{0}$, i.e., of order $c / a_{0} \approx 6 / H_{0} \approx 8 \times 10^{10} \mathrm{yr}$. Since this is several times larger than the age of the Universe, this would obviously not be a problem for massive stars. However, other dimensionful constants do exist in the model. The length scale $\ell_{0}$ entering (5.3) does not change any physical prediction at small enough distances, but within matter, all masses or coupling constants entering the matter action $S_{\text {matter }}$ may contribute to the instability timescale. Therefore, the analysis of this instability is highly nontrivial. It may actually 
happen that it does not develop at the classical level. Indeed, it is quite difficult to exhibit toy models in which the presence of ghost degrees of freedom (negative kinetic energy) imply classical instabilities, whereas they are obvious in presence of tachyons (maximum of a potential). On the other hand, ghost degrees of freedom are known to be deadly at the quantum level, because the vacuum then disintegrates instantaneously [69].

In conclusion, the nonminimally-coupled model (5.1)-(5.2) has many interesting features, notably the fact that it is rather simple and that its predictions can be easily computed from the general relativistic metric. But it is unstable and therefore one cannot trust its predictions. It may still be useful as a phenomenological framework at lowest order in powers of $\sqrt{a_{0}}$, since it goes beyond the original MOND formulation [17]. For instance, when a test particle is located between two massive bodies, it may happen that its total acceleration is smaller than $a_{0}$ although each individual force happens to be in the standard Newtonian regime $\left(f>m a_{0}\right)$. In such a case, the historical MOND theory does not clearly predict what should happen, whereas the present nonminimally-coupled model unambiguously defines the dynamics from the local curvature tensor and its covariant derivatives. This model also allows us to compute consistent MOND effects for non-spherically symmetric sources. But its main interest is to show that the MOND dynamics is consistent with most of GR's symmetries. It also illustrates in a clear way many generic problems of other MOND-like field theories of the literature, notably their unpredictiveness (because they are just tuned to reproduce the needed physics) and their almost systematic instability.

\section{B. A nonminimal scalar-tensor model}

The most serious problem of the above nonminimally-coupled model is its instability because of the presence of higher derivatives of the metric in the matter action. By introducing back a single scalar field, we will now show that this instability may be avoided (but that a more subtle consistency problem anyway remains, as discussed at the end of the present section). It will not even be necessary to give it an aquadratic kinetic term as in Sec. IIE, A standard scalar-tensor theory of the form (2.12) will suffice for our purpose, but with a more complex physical metric $\tilde{g}_{\mu \nu}$.

The idea is very similar to the previous section VA. We showed in Eqs. (5.5)-(5.6) that a combination of curvature tensors and their covariant derivatives gave us a local access to the baryonic mass $M$ and to the distance $r$, independently from each other. It was thus possible to construct from them the needed MOND potential $\sqrt{G M a_{0}} \ln r$. In standard scalar-tensor theories, with an exponential matter-scalar coupling function $A(\varphi)=e^{\alpha \varphi}$, we know that the scalar field takes the form $\varphi=-\alpha G M / r c^{2}+\mathcal{O}\left(1 / c^{4}\right)$ in the vicinity of a spherical body of mass $M$, if the background value $\varphi_{0}$ vanishes. Then it is obvious that combinations of $\varphi$ and its derivative $\partial_{r} \varphi=\alpha G M / r^{2} c^{2}+\mathcal{O}\left(1 / c^{4}\right)$ also give us access to $M$ and $r$ independently. One may thus imitate the previous model (5.1)-(5.2) by defining

$$
S=\frac{c^{4}}{4 \pi G} \int \frac{d^{4} x}{c} \sqrt{-g_{*}}\left\{\frac{R^{*}}{4}-\frac{s}{2}-\frac{1}{2}\left(\frac{m c}{\hbar}\right)^{2} \varphi^{2}\right\}+S_{\text {matter }}\left[\psi ; \tilde{g}_{\mu \nu}\right],
$$

where $s \equiv g_{*}^{\mu \nu} \partial_{\mu} \varphi \partial_{\nu} \varphi$ denotes as before the standard kinetic term of the scalar field, and 
where $\tilde{g}_{\mu \nu} \equiv A^{2} g_{\mu \nu}^{*}+B \partial_{\mu} \varphi \partial_{\nu} \varphi$ takes the disformal form (3.21) with

$$
\begin{aligned}
A(\varphi, s) & \equiv e^{\alpha \varphi}-\frac{\varphi X}{\alpha} h(X), \\
B(\varphi, s) & \equiv-4 \frac{\varphi X}{\alpha} \frac{1}{s} \\
X & \equiv \frac{\sqrt{\alpha a_{0}}}{c} s^{-1 / 4} .
\end{aligned}
$$

The function $h(X)=(1+X)^{-1}+\ln (1+X)$ was chosen in Eq. (5.8) to reproduce the MOND $1 / r$ force at large distances but a negligible one at small distances. The potential $V(\varphi)=\frac{1}{2}(m c / \hbar)^{2} \varphi^{2}$ introduced in Eq. (5.10) has a negligible influence on local physics (solar system, binary pulsars, galaxies and clusters) if the mass it involves is small enough, say $m c^{2} / \hbar \lesssim H$ where $H$ denotes the Hubble constant. However, it plays a role in the cosmological evolution of the scalar field, driving it to the minimum $\varphi_{0}=0$. This term ensures thus that the background value of the scalar field vanishes. Note however that the above model must be refined to remain consistent in the cosmological regime $(s<0)$, on the hypersurfaces $s=0$ surrounding any galaxy or cluster (see Sec. IIF above), and near the center of any massive body where $s \rightarrow 0$. It suffices, for instance, to replace $s$ by $\sqrt{s^{2}+\varepsilon^{2}}$ in Eqs. (5.11), where $\varepsilon \sim(m c / \hbar)^{2} \lesssim H^{2} / c^{2}$ is small enough to have negligible influence both on Newtonian and MONDian physics. As already underlined below Eq. (2.18) and in Sec. VA, such a refinement just illustrates how to define a consistent field theory, but there is no reason to trust its predictions in the cosmological regime $s \leq 0$. Indeed, many other functions of $s$ could connect smoothly to those entering (5.11) for small values of $s$, and they are not imposed by the MOND phenomenology.

Since the above model defines a standard scalar-tensor theory outside matter (in vacuum), we know that the scalar field takes the form $\varphi \propto-G M / r c^{2}+\mathcal{O}\left(1 / c^{4}\right)$ in the vicinity of a spherical body of mass $M$. The multiplicative factor is just $\alpha$ in scalar-tensor theories, but it may now be modified by the MOND-like corrections we added in the physical metric $\tilde{g}_{\mu \nu}$. One expects it to remain close to $\alpha$ if the matter sources are compact enough to be in the Newtonian regime of the model. This is not only the case for solar-system bodies and binary pulsars, but also for almost all the baryonic mass of galaxies and clusters. We will thus merely assume that $\varphi \approx-\alpha G M / r c^{2}$ in the present discussion. We will come back below to the (crucial) problem of the scalar field equation within matter, and thereby to the actual coupling constant $\alpha$ entering this expression. The value $\varphi \approx-\alpha G M / r c^{2}$ implies that $X=r / \sqrt{G M / a_{0}}$, which not only gives us a direct access to the radial distance $r$, but also involves the MOND characteristic radius $\ell_{0}=\sqrt{G M / a_{0}}$. This is thus already a bonus with respect to the purely metric model (5.1)-(5.2), since one needed to impose by hand that the arbitrary scale $\ell_{0}$ was much larger than the solar-system size but smaller than the radius of any galaxy. Here, this is obtained automatically from a rather simple definition of $X$, Eq. (5.11c). One also finds that $\varphi X / \alpha=-\sqrt{G M a_{0}} / c^{2}$, i.e., precisely (by construction) the needed factor of the MOND logarithmic potential. ${ }^{20}$ Therefore, the physical metric admits

20 This negative value ensures that the matter field equations are well posed, since inequalities (3.22) are satisfied: $A^{2}+s B=A^{2}+4 \sqrt{G M a_{0}} / c^{2}>A^{2}>0$. 
the following post-Newtonian expansion:

$$
\tilde{g}_{\mu \nu}=\left[1-\frac{2 \alpha^{2} G M}{r c^{2}}+\frac{2 \sqrt{G M a_{0}}}{c^{2}} h\left(\frac{r}{\sqrt{G M / a_{0}}}\right)\right] g_{\mu \nu}^{*}+\frac{4 \sqrt{G M a_{0}}}{c^{2}} \delta_{\mu}^{r} \delta_{\nu}^{r}+\mathcal{O}\left(\frac{1}{c^{4}}\right) .
$$

For distances $r \gg \sqrt{G M / a_{0}}$, the function $h(X)$ has been chosen to reproduce the MOND logarithmic potential, and the disformal contribution $\propto \delta_{\mu}^{r} \delta_{\nu}^{r}$ in (5.12) is precisely the one needed to reproduce the same light deflection as GR in presence of dark matter, Eq. (3.24), as already noticed below Eq. (5.9) for the previous nonminimal metric model. On the other hand, for $r \ll \sqrt{G M / a_{0}}$, the function $h(X)$ tends to 1 and does not contribute to any physical observable, whereas the disformal contribution $\propto \delta_{\mu}^{r} \delta_{\nu}^{r}$ still gives the constant anomalous light deflection (3.24), but too small to be of observational significance in the solar system. Therefore, this model does reproduce the right phenomenology. Solar-system tests just impose the tight bound $\alpha^{2}<10^{-5}$, as in standard scalar-tensor theories, because the $-2 \alpha^{2} G M / r c^{2}$ term contributes to our interpretation of light deflection, as explained in Eq. (3.4) above.

Since this model has been devised to reproduce the standard Brans-Dicke predictions for distances $r \ll \sqrt{G M / a_{0}}$, we can also conclude that it passes binary-pulsar tests, which impose the constraint $\alpha^{2}<4 \times 10^{-4}[124,125,126]$, weaker than the above solar-system one. Contrary to our discussion of the TeVeS model in Sec. IVD, the (spin-2 and spin0) gravitational waves are now strictly the same as in Brans-Dicke theory, even at large distances where MOND effects can be observed on material test bodies. Indeed, this model is a pure scalar-tensor theory in vacuum, so that gravitational waves themselves do not feel any MONDian effect. This is an interesting feature of this model, and also its main difference with the previous ones proposed in the literature. In previous models, the helicity0 (scalar) waves undergo MONDian effects at large distances from massive sources, whereas they follow the same geodesics (of the Einstein metric $g_{\mu \nu}^{*}$ ) as the helicity-2 waves in the present model. Unfortunately, this different prediction cannot be used to experimentally discriminate between these models, because the solar-system constraint $\alpha^{2}<10^{-5}$ on the matter-scalar coupling constant implies that gravitational-wave detectors (made of matter) are almost insensitive to scalar waves. On the other hand, let us underline that they could in principle discriminate between such MOND-like field theories and the standard dark matter hypothesis. Indeed, all RAQUAL and disformal models, including TeVeS and the present one, predict that the helicity- 2 waves are not directly coupled to the scalar field, and thereby are not deflected by what we usually interpret as dark matter haloes, contrary to light (coupled to the physical metric $\tilde{g}_{\mu \nu}$ describing MONDian effects). Therefore, when the LISA interferometer will detect gravitational waves emitted from optically known sources, their direction should be slightly different from the optical direction if there exists at least one intermediate gravitational deflector. Unfortunately again, the LISA angular resolution will be about the angular size of the Moon (30 arcmin), much too large to measure such a deflection angle between electromagnetic and gravitational waves. But the time-delay between gravitational waves and optical or neutrino pulses from supernovae would allow us to discriminate between MOND and the dark matter hypothesis, as recently shown in [131].

The deadly problem of the previous model (5.1)-(5.2) was its Ostrogradskian instability. One may thus wonder if the present scalar-tensor variant avoids it. Indeed, since the matter action a priori involves covariant derivatives of the matter fields, it depends on the first derivatives of the physical metric $\tilde{g}_{\mu \nu}$, and thereby of the second derivatives of $\varphi$, see 
Eqs. (5.11). The same instability as before, caused by second and higher derivatives, seems thus to spoil the present model too. However, let us recall that the standard model of particle physics only involves gauge bosons described by 1-forms, and fermions described by Dirac spinors. If is well known that all covariant derivatives may be replaced by ordinary ones in forms, because their antisymmetry cancels all the Christoffel symbols $\tilde{\Gamma}_{\mu \nu}^{\lambda}$. Therefore, there is actually no derivative of $\tilde{g}_{\mu \nu}$ involved in the action describing the dynamics of gauge bosons, and only $\varphi$ and its first gradient $\partial_{\mu} \varphi$ enter it. On the other hand, the action of a spinor in curved spacetime does depend of the first derivative of the metric (via the derivative of a tetrad, cf. Sec. 12.5 of [117]), but only linearly. Schematically, it reads $\bar{\psi} \tilde{g}^{m} \not \partial \psi \bar{\psi} \tilde{g}^{n}(\not \partial \tilde{g}) \psi$. Therefore, $\nabla_{\mu}^{*} \partial_{\nu} \varphi$ enters this action, but if one tries to define a conjugate momentum $p_{2} \equiv \partial \mathcal{L} / \partial \ddot{\varphi}$, then its expression does not depend on any second derivative of $\varphi$. Therefore, one cannot express $\ddot{\varphi}$ as a function of $\varphi, \dot{\varphi}$ and $p_{2}$ as in Eq. (2.4), and we are thus precisely in the case of a degenerate Lagrangian for which the Ostrogradskian instability does not occur. More explicitly, all second time derivatives of the scalar field, $\ddot{\varphi}$, may be eliminated by partial integration. In conclusion, the action of the standard model of particle physics actually does not depend on $\ddot{\varphi}$, and the above model is thus free of Ostrogradskian instability.

However, this absence of generic instability does not suffice to prove that the model is indeed stable. One must also check that the scalar field equation remains consistent within matter. In the most general case of arbitrary matter fields and arbitrary initial conditions, this problem is quite involved because the matter action depends on the derivative of the scalar field. As discussed in Sec. IIIC, one should write all the field equations, including for matter, and prove that the second derivatives of the scalar field define a hyperbolic operator. The simpler case of a pressureless perfect fluid will however suffice to exhibit the subtle difficulties appearing in this class of models. The generalized RAQUAL kinetic term

$$
\tilde{f}\left(\varphi, s, \partial_{0} \varphi\right)=s+\frac{8 \pi G \bar{\rho}}{c^{2}} A \sqrt{1-\left(\partial_{0} \varphi\right)^{2} B / A^{2}},
$$

written here in the rest frame of the perfect fluid and in coordinates locally diagonalizing the Einstein metric $g_{\mu \nu}^{*}=\operatorname{diag}(-1,1,1,1)$, should satisfy inequalities (a1) and (b1) quoted below Eq. (3.27). The contribution of the scalar field to the Hamiltonian should also be bounded by below. Since the model has been constructed to reproduce the MOND dynamics at the lowest (Newtonian) order, without imposing its behavior at higher post-Newtonian orders nor in the cosmological regime, let us focus on the conditions (a2), (b2) and (c2) that $\tilde{f}$ should at least satisfy at the Newtonian level. As mentioned in Sec. IIIC, condition (c2) is actually implied by (a2), because we imposed a positive value of the coupling function $B$ in order to increase light deflection in the MOND regime. Moreover, one can check easily that the terms proportional to $\left(\partial_{0} \varphi\right)^{2}$ may be neglected in (a2) and (b2). On the other hand, note that $A^{\prime} / c^{2}$ and $s A^{\prime \prime} / c^{2}$ are actually of Newtonian order $\mathcal{O}\left(1 / c^{0}\right)$, in spite of the explicit factor $1 / c^{2}$. At this Newtonian order, it suffices thus to check conditions

(a2) $\tilde{f}^{\prime}>0$

(b2) $2 s \tilde{f}^{\prime \prime}+\tilde{f}^{\prime}>0$,

where $\tilde{f}$ may be approximated as $\tilde{f} \approx s+\left(8 \pi G \bar{\rho} / c^{2}\right) A$, as if we were considering a RAQUAL model in vacuum; see conditions (a) and (b) of Sec. IIE. In such a case, these hyperbolicity conditions suffice to ensure that the scalar-field contribution to the Hamiltonian is bounded 
by below. [The coupling function $B$ plays an even more negligible role in the Hamiltonian than in the hyperbolicity conditions, namely of order $\mathcal{O}\left(1 / c^{4}\right)$ smaller than the lowest term involving the matter density $\bar{\rho}$.]

In the Newtonian regime, i.e., when the MONDian correction involving $\varphi X / \alpha$ in Eqs. (5.11) are negligible, it is obvious that the hyperbolicity conditions (a2) and (b2) are satisfied. The model reduces indeed to the standard Brans-Dicke theory, whose consistency is well known. If all matter in the Universe was clustered as compact enough bodies, like stars and planets, then this Newtonian regime of the model would always be reached when considering the scalar field equation within matter, and its Cauchy problem would always be well posed. As mentioned below Eqs. (5.11), there would still remain a difficulty near the center of any body, where the spatial gradient of the scalar field tends to 0 , and therefore where MOND corrections may become large (before they are saturated by our ad hoc replacement of $s$ by $\sqrt{s^{2}+\varepsilon^{2}}$ ). However, it is not even necessary to study such centers of compact bodies to point out an extremely serious difficulty of this model. Indeed, there does exist dilute gas in the outer regions of galaxies where the MOND phenomenology applies. One should therefore check whether conditions (a2) and (b2) hold when the $\varphi X / \alpha$ corrections start dominating even over the Newtonian force. Actually, for the coupling functions (5.11), one finds that condition (b2) is easily satisfied, but it happens that (a2) is not. Indeed, $\tilde{f}^{\prime} \approx 1+\left(8 \pi G \bar{\rho} / c^{2}\right) A^{\prime}$, where $A^{\prime}=(h+X d h / d X) \varphi X / 4 \alpha s$. The coefficient $(h+X d h / d X)$ varies from 1 to 3.3 in the range of distances relevant to the MOND regime, namely $X=r / \sqrt{G M / a_{0}}<10$. On the other hand, $\varphi X / \alpha s=-\left(\sqrt{G M a_{0}} / c^{2}\right)\left(\alpha G M / r^{2} c^{2}\right)^{-2}$ is a huge negative number, because the kinetic term $s$ of the scalar field appears in its denominator. Even multiplied by the small gas density $G \bar{\rho} / c^{2}$ existing in the outer regions of galaxies, this negative contribution to $\tilde{f}^{\prime}$ dominates by several orders of magnitude over the 1 coming from the standard kinetic term in vacuum. In other words, $\tilde{f}^{\prime}<0$ within such a gas, and the scalar perturbations are thus ill defined. In spite of its nice features and its relative simplicity, the model (5.11) is thus inconsistent, because the scalar field equation is not always hyperbolic within matter. Such a difficulty was not discussed in previous works on MOND-like field theories, although it seems generic for disformally-coupled scalar-tensor models (3.21).

Of course, one may try to tune the coupling function $A$, Eq. (5.11a), to cure this inconsistency. For instance, since $\varphi X / \alpha$ takes the constant value $-\sqrt{G M a_{0}} / c^{2}$ outside matter, one may add to $A$ any function of $\varphi X / \alpha$ without changing its spatial gradient, i.e., the force felt by a test mass. However, it is straightforward to show that there is not much freedom for such a function. In order to ensure condition (a2) without spoiling (b2), it must be close to $k(\varphi X / \alpha)^{-2}$, where $k$ is a constant. This precise expression has the peculiarity not to contribute to condition (b2), whereas it adds a positive contribution to $\tilde{f}^{\prime}$. The crucial difficulty is that the proportionality constant $k$ must take a fixed value, imposed in the action of the model, and should not be chosen independently for each galaxy or cluster (cf. our discussion in Sec. IIB above). To guarantee condition (a2) in any situation, $k$ should thus be chosen large enough, and one finds that $k>2\left(G M_{\text {cluster }} a_{0} / c^{4}\right)^{3 / 2}$ would suffice, where $M_{\text {cluster }}$ is the largest cluster mass existing in the Universe. Obviously, this appearance of another dimensionful constant in the model underlines that it would be even more fine tuned than before. [One might be tempted to simply set $k=1$ to make the model more natural, but the coupling constant $A$ would then take large values instead of admitting a post-Newtonian expansion $A=1+\mathcal{O}\left(1 / c^{2}\right)$, and the predicted MONDian effects would then be reduced by the inverse of this large value.] But it happens that such a tuning of the coupling function $A$ actually 
does not work. Indeed, although a term $k(\varphi X / \alpha)^{-2}$ would not contribute to the force felt by a test mass, it does contribute to the scalar field equation $2 \nabla_{\mu}^{*}\left(\tilde{f}^{\prime} \nabla_{*}^{\mu} \varphi\right)=\partial \tilde{f} / \partial \varphi$. In the MOND regime, it even dominates over the other contributions in both sides of this field equation, in spite of its coefficient $\left(8 \pi G \bar{\rho} / c^{2}\right)$ involving the small gas density $\bar{\rho}$. In a region where $\bar{\rho}$ is almost constant, one may thus divide each side by it, and one gets a scalar field equation fully different from the one satisfied in vacuum, ${ }^{21}$ although it does not depend any longer on the precise value of $\bar{\rho}$. Its exact solution is difficult to find, but the signs involved anyway show that $\varphi$ would quickly tend to 0 within matter (in the MOND regime), instead of keeping the form $\varphi \approx-\alpha G M / r c^{2}$ that we assumed from the beginning of this section to construct the present model. Even without invoking the signs on the different terms in this field equation, it is anyway obvious to check that $\varphi \approx-\alpha G M / r c^{2}$ cannot be valid within the gas in outer regions of a galaxy. In other words, the extra force predicted by such a model, if any, would not take the MOND form that we wished. In conclusion, a tuning of the coupling function $A$ to ensure the hyperbolicity of the scalar field equation within matter suffices to ruin the predictions of the model.

The above hyperbolicity problem underlines again how difficult it is to construct a MOND-like field theory, even fine tuned. In spite of the presence of several free functions in the model, which could a priori fit any phenomenology one wishes, the consistency of the field equations within matter is a strong enough condition to rule it out. One should however underline that this conclusion cannot be considered as a no-go theorem. The same framework as Eqs. (5.10)-(5.11) might be able to reproduce consistently the MOND dynamics with different coupling functions $A$ and $B$. If the scalar field does not take the form $-\alpha G M / r c^{2}$ in the MOND regime, then specific combinations of $\varphi$ and its kinetic term $s$ might be able to give us access independently to the crucial factor $\sqrt{G M a_{0}} / c^{2}$ and to the radius $r / \sqrt{G M / a_{0}}$. The MOND metric (5.12) might thus be obtained again, and our discussion above shows that the Ostrogradskian instability would still be avoided. We expect the hyperbolicity conditions (a2) and (b2) to still cause serious problems within matter in the MOND regime, but we do not have any proof. Our investigations indicate anyway that the coupling functions $A$ and $B$ would very probably involve unnaturally complicated expressions (like large powers of $s$ or transcendental functions).

\section{The Pioneer anomaly}

As mentioned in Sec. II the two Pioneer spacecrafts exhibited an anomalous extra acceleration $\delta a \approx 8.5 \times 10^{-10} \mathrm{~m} . \mathrm{s}^{-2}$ towards the Sun, between 30 and 70 AU [10, 132, 133]. From a theoretical point of view, this anomaly is a priori much easier to explain than dark matter by a fine-tuned model modifying Newtonian gravity. Indeed, it has been observed in our single solar system, mainly characterized by the solar mass $M_{\odot}$, whereas galaxy and cluster rotation curves have confirmed the relation $M_{\text {dark }} \propto \sqrt{M_{\text {baryon }}}$ for many different values

21 This problem actually already arises with the initial coupling function $A$, Eq. (5.11a), whose MONDian terms dominate within the gas in outer regions of galaxies. However, one can check that the scalar field and its derivative keep the same orders of magnitude as the vacuum (Brans-Dicke) behavior $\varphi \approx-\alpha G M / r c^{2}$ we assumed, so that the phenomenological predictions remain correct up to factors of order unity. On the other hand, the scalar field behavior is fully changed within this outer gas when the correcting term $k(\varphi X / \alpha)^{-2}$ is included. 
of the masses. A possible explanation (or, rather, description) of the Pioneer acceleration would be to couple matter to a scalar field, whose potential is merely fitted to reproduce observed data. However, in order not to spoil the precision tests of Kepler's third law in the solar system, such a potential should not manifest at distances smaller than Saturn's orbit $(\approx 10 \mathrm{AU})$, and cause an almost constant extra force beyond $30 \mathrm{AU}$. Besides the fact that this would be quite unnatural, one may also wonder if this would correspond to a stable theory. Indeed, the simplest stable potential for a scalar field is a mass term $\propto \varphi^{2}$, and it causes a force which decreases (exponentially) as the distance grows. Even within the a priori simpler case of the Pioneer anomaly, the construction of a stable model does not seem obvious, thus.

A first, important, step has been understood in the series of papers [134, 135, 136] by Reynaud and Jaekel. From a phenomenological viewpoint, it is much more natural to describe the Pioneer anomaly by a modification of the spatial metric $g_{i j}$ rather than the Newtonian potential involved in $g_{00}$. Indeed, if the metric is written in the Schwarzschild form (3.12), then the geodesics equation for a test mass $m_{0}$ reads

$$
\mathcal{E}^{2} / c^{2}-\left(m_{0}^{2} c^{2}+\mathcal{J}^{2} u^{2}\right) \mathcal{B}=\mathcal{J}^{2} \mathcal{A B} u^{\prime 2}
$$

where $\mathcal{E}$ is the particle conserved energy (including rest mass $m_{0} c^{2}$ ), $\mathcal{J}$ its angular momentum, $u \equiv 1 / r$, and a prime denotes here derivation with respect to the angle in polar coordinates. For a circular orbit, $u^{\prime}=0$ so that the spatial metric component $g_{r r}=\mathcal{A}$ does not enter this equation. ${ }^{22}$ More generally, its right-hand side is proportional to the square of the orbit's eccentricity, $e^{2}$. In conclusion, planets on nearly circular orbits are almost insensitive to a small modification of $\mathcal{A}$, whereas they would feel any extra potential entering the time component $\mathcal{B}$. On the other hand, hyperbolic trajectories, ${ }^{23}$ like those of light rays and the Pioneer spacecrafts, are directly sensitive to a modification of $\mathcal{A}$. This is actually the crucial difference between galaxy rotation curves (which exhibit a discrepancy with Newton's law for the circular orbits of outer stars) with the Pioneer anomaly (occurring for hyperbolic trajectories). While it was necessary to modify the time-time metric $g_{00}$ to reproduce the MOND phenomenology (together with the spatial one $g_{i j}$ to predict the correct light deflection), it is possible to account for the Pioneer anomaly by a modification of $g_{r r}$ alone.

However, post-Newtonian tests in the solar system, notably those of light deflection and Shapiro time delay, severely constrain the magnitude of the deviations from general relativity in the spatial component $\mathcal{A}$. In particular, it is impossible to account for the Pioneer anomaly

${ }^{22}$ Beware that in the Schwarzschild coordinates we are using, the post-Newtonian term in the time component $g_{00}$ of the metric is proportional to the combination $\beta-\gamma$ of the Eddington parameters, whereas it is proportional to $\gamma$ in the radial component. It would thus be misleading to claim that circular orbits are merely independent of $\gamma$. The correct statement should underline that this is only true for a fixed value of $\beta-\gamma$.

${ }^{23}$ Recall that the virial theorem implies $v^{2} / c^{2}=\mathcal{O}\left(G M / r c^{2}\right)$ for bound orbits, whereas hyperbolic ones reach a nonzero asymptotic velocity $v^{2} / c^{2} \gg G M / r c^{2}$ for large distances $r$. As compared to the timetime contribution $g_{00} c^{2} d t^{2}$ to the line element $d s^{2}$, the spatial one $g_{i j} d x^{i} d x^{j}=g_{i j} v^{i} v^{j} d t^{2}$ is thus of post-Newtonian order for bound orbits, but much larger for hyperbolic ones (and actually of the same order as the time-time contribution for light rays, yielding the famous $(1+\gamma)$ factor of the light deflection formula). 
within the parametrized post-Newtonian formalism [1, 137, 138, 139, 140, 141, 142], which describes all metrically-coupled theories of gravity at order $\mathcal{O}\left(1 / c^{2}\right)$ with respect to the Newtonian force, under the hypothesis that the gravitational field does not involve any characteristic length scale. This hypothesis implies that all post-Newtonian parameters, including the famous Eddington parameters $\beta$ and $\gamma$, must be independent of the radial distance $r$. Within this wide class of theories, the Pioneer anomaly is merely inconsistent with other precision tests in the solar system.

The lesson of Refs. [134, 135, 136] is that a spatial dependence of the Eddington parameter $\gamma$, going thus beyond the PPN formalism, suffices to account for the Pioneer anomaly without spoiling the classic tests. This parameter should of course remain very close to the general relativistic value $\gamma=1$ at small radii, so that light-deflection and time-delay predictions are consistent with observation, but it may change at distances $\gtrsim 30 \mathrm{AU}$ to fit Pioneer data. In their first analysis, Refs. [134, 135] showed that an expression $\gamma=1+\kappa r^{2}$ would a priori suffice, for an appropriate constant $\kappa$ tuned to reproduce the Pioneer extra acceleration. In other words, the radial component of the metric should take the form $g_{r r}=1+2 \gamma G M_{\odot} / r c^{2}+$ $\mathcal{O}\left(1 / c^{4}\right)=1+\left(2 G M_{\odot} / c^{2}\right)(1 / r+\kappa r)+\mathcal{O}\left(1 / c^{4}\right)$, in which a small term linear in $r$ corrects the general relativistic $1 / r$ potential. The refined discussion of Ref. [136] actually leads to a more complex radial dependence of the parameter $\gamma$, cubic in $r$, but the qualitative conclusion remains the same.

Let us just summarize here the simpler result of [134, 135]. The extra acceleration $\delta \mathbf{a}$ caused by a term $k_{n} r^{n}$ added to $g_{r r}=\mathcal{A}$ is a straightforward consequence of the geodesics equation (5.14). One finds

$$
\delta \mathbf{a}=-\frac{1}{2} k_{n} n r^{n-1} v^{2} \mathbf{n},
$$

where $v$ denotes the particle's velocity and $\mathbf{n} \equiv \mathbf{x} / r$ the radial unit vector (pointing away from the Sun). Since the velocities of the Pioneer spacecrafts were almost constant on the range of distances where their anomalous acceleration was observed, one thus needs $n=1$ and a positive value of $k_{1}$ to obtain a constant $\delta \mathbf{a}$ directed towards the Sun. Actually, one does not directly observe the acceleration of the spacecrafts, but deduce it from Doppler tracking. One should thus also take into account the fact that electromagnetic waves travel in the same metric, and feel the anomalous potential too. Reference [134, 135] shows that this leads to a factor 2 difference in the prediction of the recorded anomalous acceleration, i.e., $\delta a=k_{1} v^{2}$. Numerically, for the Pioneer spacecrafts, $v \approx 1.2 \times 10^{4} \mathrm{~m} . \mathrm{s}^{-1}$ and $\delta a \approx$ $8.5 \times 10^{-10} \mathrm{~m} \cdot \mathrm{s}^{-2}$, therefore

$$
k_{1} \approx \frac{0.5 \mathrm{~m} \cdot \mathrm{s}^{-2}}{c^{2}} \approx\left(10^{6} \mathrm{AU}\right)^{-1}
$$

If interpreted as a varying Eddington parameter $\gamma=1+\kappa r^{2}$, with $2 \kappa G M_{\odot} / c^{2}=k_{1}$, this gives $\kappa \approx 45 \mathrm{AU}^{-2}$. The refined analysis of Ref. [136] yields a quadratic extra potential $k_{2} r^{2}$ with $k_{2} \approx 8 \times 10^{-8} \mathrm{AU}^{-2}$, i.e., a varying Eddington parameter $\gamma \approx 1+4(\mathrm{r} / \mathrm{AU})^{3}$. It further tunes this extra potential in the form $k_{2}\left(r-r_{\oplus}\right)^{2}+k_{2}^{\prime}\left(r-r_{\oplus}\right)$, where $r_{\oplus}$ is the radius of the Earth's orbit and $k_{2}^{\prime}$ another constant characterizing the anomalous acceleration at $r=r_{\oplus}$. We shall not take into account such refinements in the following, but just mention that they would not change our conclusion.

The last difficulty is to exhibit a consistent field theory reproducing such a radial dependence of $\gamma$, both stable and admitting a well-posed Cauchy problem. Because of the generic difficulties with modified gravity models recalled in Sec. III, and notably the stability issues 
of higher-order gravity discussed in Sec. IIC, it is a priori not obvious that such a theory exists, and Refs. [134, 135] actually write nonlocal field equations without discussing these crucial mathematical requirements. But it happens that the scalar-tensor framework of Sec. $\mathrm{VB}$ above suffices to reproduce consistently a varying Eddington parameter $\gamma$. Indeed, the disformal contribution $B \partial_{\mu} \varphi \partial_{\nu} \varphi$ to the physical metric (3.21) is precisely what is needed to change only the radial component $g_{r r}$, i.e., what is denoted as $\mathcal{A}$ in the geodesics equation (5.14). As in Sec. VB, one may thus consider a scalar field weakly coupled to matter via a Brans-Dicke conformal factor $A^{2}(\varphi)=\exp (2 \alpha \varphi)$, and build the appropriate correction ${ }^{24}$ to the radial metric by using $\varphi \approx-\alpha G M_{\odot} / r c^{2}$ and $\sqrt{s}=\partial_{r} \varphi \approx \alpha G M_{\odot} / r^{2} c^{2}$. Since we do not have any experimental evidence that the Pioneer anomalous acceleration is related to the solar mass (nor its square root nor any function of it), several expressions can actually be used to build an extra gravitational potential linear in $r$, for instance $1 / \varphi$ or $s^{-1 / 4}$, or any function like $\varphi^{-1} f\left(\varphi^{4} / s\right)$. The simplest choice would be to impose $B=-\lambda_{5} / \varphi^{5}$, where $\lambda_{5}$ is a positive constant, i.e., a physical metric of the form

$$
\tilde{g}_{\mu \nu}=e^{2 \alpha \varphi} g_{\mu \nu}^{*}-\frac{\lambda_{5}}{\varphi^{5}} \partial_{\mu} \varphi \partial_{\nu} \varphi
$$

so that $\tilde{g}_{r r}=e^{2 \alpha \varphi} g_{r r}^{*}-\left(\lambda_{5} / \varphi\right)\left(s / \varphi^{4}\right)$ involves a positive correction proportional to $r$. [The more complex, quadratic, potential derived in Ref. [136] can be obtained in the same way, by choosing for instance $B=\mu_{6} / \varphi^{6}+\mu_{5} / \varphi^{5}+\mu_{4} / \varphi^{4}$, where $\mu_{4,5,6}$ are three constants such that $B>0$, in order to predict an extra acceleration directed towards the Sun.] Of course, expression (5.17) is valid only in the vicinity of the Sun, where $s>0$ and $\varphi<0$. It should be refined to remain valid in other regimes, but the Pioneer data do not tell us how. Since the condition $A^{2}+s B>0$ must be satisfied to ensure the hyperbolicity of the matter field equations, see Eq. (3.22), it might be better to consider coupling functions $B=\lambda_{9} s /|\varphi|^{9}$ or $B=\lambda_{1} /(|\varphi| s)$. However, it would also suffice to multiply the above $B=-\lambda_{5} / \varphi^{5}$ by a function $h(s)$, such that $h(s)=0$ for $s<0$ and $h(s)=1$ for $s>\left(\alpha G M_{\odot} / r_{\max }^{2} c^{2}\right)^{2}$, where $r_{\max } \approx 70 \mathrm{AU}$ is the largest distance for recorded Pioneer data. The above metric (5.17) should also be refined to avoid divergences when $\varphi$ passes through zero with a nonvanishing derivative. A possible solution would be to replace $-\varphi$ by $\sqrt{\varphi^{2}+\varepsilon^{2}}$, where $\varepsilon \ll \alpha G M_{\odot} / r_{\max } c^{2}$ is a tiny dimensionless number, negligible for the Pioneer phenomenology but eliminating our anomalous potential at larger distances. Numerically, one would need $\varepsilon \ll 10^{-10} \alpha<3 \times 10^{-13}$, if one takes into account the solar-system constraints on the matter-scalar coupling constant $\alpha<3 \times 10^{-3}$. On the other hand, to reproduce the observed Pioneer anomaly, the constant $\lambda_{5}$ entering Eq. (5.17) should take the numerical value

$$
\lambda_{5} \approx \alpha^{3}\left(10^{-4} \mathrm{~m}\right)^{2}<\left(2 \times 10^{-8} \mathrm{~m}\right)^{2},
$$

the upper limit corresponding to the largest allowed value for $\alpha$. [The other possible expressions for $B$ quoted above would need factors $\mu_{6} \approx \alpha^{4}\left(4 \times 10^{-9} \mathrm{~m}\right)^{2}<\left(4 \times 10^{-14} \mathrm{~m}\right)^{2}$, $\lambda_{9} \approx \alpha^{5}(0.4 \mathrm{~m})^{4}<\left(3 \times 10^{-4} \mathrm{~m}\right)^{4}$ and $\lambda_{1} \approx 10^{-14} \alpha<3 \times 10^{-17}$ respectively.]

Such numerical constants underline that we are merely fine-tuning a model to account for experimental data. However, the crucial point that we wish to stress is that the model

\footnotetext{
${ }^{24}$ A correction to the time-time metric $\tilde{g}_{00}$ would also be easy to define by using $\varphi$ and $\partial_{r} \varphi$. This might be necessary to avoid too large modifications of the planets' perihelion shifts, which depend on both of the PPN parameters $\beta$ and $\gamma$.
} 
(5.10) with a physical metric (5.17) is stable and admits a well-posed Cauchy problem. The hyperbolicity conditions (a1) and (b1) of Sec. IIIC are indeed trivially satisfied, because both coupling functions $A=\exp (\alpha \varphi)$ and $B=-\lambda_{5} / \varphi^{5}$ are independent of the kinetic term $s$, and because $B>0$ in the regime where this model is defined. Actually, for such a simple case, one may even check the hyperbolicity of the fully general scalar field equation (3.25) within any kind of matter. The fact that the Hamiltonian is bounded by below is also an obvious consequence of the positivity of $B$. [Note that all these consistency conditions are also obvious for the more complex expression $B=\mu_{6} / \varphi^{6}+\mu_{5} / \varphi^{5}+\mu_{4} / \varphi^{4}$ mentioned above to reproduce the quadratic potential derived in Ref. [136]. On the other hand, they are less trivial for the other possible expressions for $B$ quoted above, such as $B=\lambda_{9} s /|\varphi|^{9}$ or $B=\lambda_{1} /(|\varphi| s)$. However, they may still be easily checked at the lowest post-Newtonian level, where the hyperbolicity conditions reduce to (a2), (b2) and (c2) of Sec. IIIC, and where all terms proportional to $u_{*}^{\mu} \partial_{\mu} \varphi$ may be neglected.]

The final consistency check is to show that the scalar field takes indeed the form $\varphi \approx$ $-\alpha G M_{\odot} / r c^{2}$ in the solar system, since we assumed this expression to build our anomalous potential. One just writes the scalar field equation within matter, $2 \nabla_{\mu}^{*}\left(\tilde{f}^{\prime} \nabla_{*}^{\mu} \varphi\right)=\partial \tilde{f} / \partial \varphi$, where $\tilde{f}$ is defined in Eq. (3.27). If the Sun were an isolated body, the scalar field would be generated by it only, and one would have $u_{*}^{\mu} \partial_{\mu} \varphi=0$ strictly (up to a negligible time variation of the scalar field possibly imposed by the cosmological evolution). In such a case, the model reduces to Brans-Dicke theory, and one gets thus obviously $\varphi \approx-\alpha G M_{\odot} / r c^{2}$. When considering other bodies in the solar system, the largest effects due to the disformal coupling function $B$ occur at large distances from the Sun. One finds that the coupling constant $\alpha$ is then renormalized into $\alpha+\mathcal{O}\left(r v^{2} / G M_{\odot}\right) k_{1} r / \alpha$, where $k_{1} \approx\left(10^{6} \mathrm{AU}\right)^{-1}$ is the coefficient (5.16) of the linear anomalous potential we added to the radial metric. The coefficient $\mathcal{O}\left(r v^{2} / G M_{\odot}\right)$ is of order unity for planets because of the virial theorem, and solarsystem tests impose $\alpha^{2}<10^{-5}$. For the largest allowed value of $\alpha$, we can thus conclude that our anomalous potential does not contribute significantly to the scalar field equation up to $r \sim 10 \mathrm{AU}$ (i.e., Saturn's orbit). For outer planets, $\alpha$ may be increased by a factor $\lesssim 4$, but since their masses are negligible with respect to that of the Sun, the approximate value of the scalar field near the Pioneer spacecrafts remains anyway $\varphi \approx-\alpha G M_{\odot} / r c^{2}$. The largest modification of the matter-scalar coupling constant occurs for the Pioneer spacecrafts themselves (or any object on a hyperbolic orbit at similar distances from the Sun), since $r v^{2} / G M_{\odot} \approx 10$ for $r=70 \mathrm{AU}$ and the spacecrafts' velocity $v \approx 1.2 \times 10^{4} \mathrm{~m} . \mathrm{s}^{-1}$. This means that this coupling constant is increased by a factor $\sim 80$ at such distances, explaining incidentally why these spacecrafts are sensitive to the scalar field. But the local perturbation of $\varphi$ they induce does not act on their own trajectory, and it remains negligibly small for other bodies (as if we were considering 80 spacecrafts instead of one, but weakly coupled to the scalar field as in Brans-Dicke theory).

In conclusion, although the above model (5.10)-(5.17) is not justified by any underlying symmetry principle, and should be considered more as a fit of Pioneer data than as a predictive theory, it proves that it is possible to account for this anomalous acceleration in a consistent field theory, while satisfying all other solar-system data (and binary-pulsar tests too, since the model reduces to Brans-Dicke theory in vacuum). Let us recall that there is no reason to trust Eq. (5.17) in a different regime than the one relevant to the Pioneer spacecrafts, and notably in cosmology. Note also that there exist many different ways to reproduce the same phenomenology, as exhibited by various expressions of the disformal coupling function $B$ above. Our only addition with respect to Refs. [134, 135, 136] is that 
a varying post-Newtonian parameter $\gamma$ is indeed possible within a stable model admitting a well-posed Cauchy problem.

\section{CONCLUSIONS}

One of the aims of this article was to clarify the mathematical consistency of various field theories proposed in the literature to reproduce the MOND phenomenology. We underlined that besides experimental constraints which need to be satisfied, including new ones that we discussed (notably binary-pulsar tests), there are also several basic requirements that a field theory needs to meet: stability (i.e., boundedness by below of its Hamiltonian) and well-posedness of its Cauchy problem (i.e., notably the fact that all field equations must be hyperbolic). In our opinion, the TeVeS model [30, 31] is presently the most promising, but it also presents several serious difficulties discussed in Sec. IV, and cannot be considered yet as a fully consistent theory. We examined another possible route to reproduce the MOND phenomenology, within the class of the already studied RAQUAL models [27], but with a different spirit. Its originality is that the theory is particularly simple in vacuum (pure general relativity or Brans-Dicke theory). However, the analysis of the field equations within matter exhibited a deadly inconsistency. Although this framework did not provide any serious alternative to TeVeS yet, we anyway believe further work along its line might do so. Its interest also lies in its relative simplicity. The model proposed in Sec. VA, assuming a nonminimal coupling of matter to the spacetime curvature, is for instance an excellent toy model of other MOND-like field theories, as its exhibits their generic difficulties: nonpredictiveness, fine-tuning, and above all instability.

It should be underlined that other experimental problems also need to be addressed by any field theory of MOND, although we did not discuss them in the present article. First of all, weak lensing observations have already exhibited several evidences of dark matter not located around clusters of baryonic matter, notably the famous bullet cluster [143] and the very recent dark matter ring [144]. The basic idea of the MOND dynamics seems therefore to be contradicted. However, several studies [145, 146, 147, 148] have argued that such observations do not rule out MOND-like field theories, whose predictions can differ significantly from the original MOND proposal in non-spherical and dynamical situations. Another generic problem of models avoiding the dark matter hypothesis is to predict the right relative heights of the second and third acoustic peaks of the CMB spectrum. Indeed, in absence of collisionless dark matter, their heights should decrease monotonically by Silk damping, whereas WMAP data [3, 4] confirm that the second and third peaks have similar heights (consistent with the dark matter paradigm). Reference [149] analyzed the CMB spectrum within the TeVeS model, and found that it can fit experimental data provided their is a present density $\Omega_{\nu} \approx 0.17$ of massive neutrinos. This number is not far from the needed dark matter density $\Omega_{\mathrm{DM}} \approx 0.24$ in the standard $\Lambda \mathrm{CDM}$ (cosmological constant plus cold dark matter) cosmological model, and illustrates that the existence of dark matter (as massive neutrinos) would anyway be needed in the TeVeS model, although the MOND dynamics was initially devised to avoid the dark matter hypothesis. However, it is already known that some amount of dark matter is anyway needed to account for the cluster rotation curves, within the MOND model, and massive neutrinos would be perfect candidates since they can cluster on such scales although they are too light to cluster on galaxy scales. Therefore, a MOND-like field theory remains a possibility, provided there is a large enough density of massive neutrinos — or any other dark matter candidate with similar properties. 
In the model [33], closely related to TeVeS, one of the two scalar degrees of freedom is assumed to play the role of this dark matter. A third difficulty of MOND-like field theories is also to explain the numerical coincidence $a_{0} \approx \mathrm{cH}_{0} / 6$ between the MOND acceleration constant and the Hubble constant (i.e., the present expansion rate of the Universe) [17]. Some very promising ideas have been developed notably in [33], but it cannot be considered as a prediction. For instance, it may happen that $a_{0}$ is actually related to the cosmological constant instead of the Hubble expansion rate, via a relation like $a_{0} \approx \sqrt{\Lambda c^{2} / 72}$. In such a case, it would not vary with time, and one would "only" have to explain why it happens to take this precise value. On the other hand, a constant value of $a_{0}$ would probably be ruled out by the observed CMB spectrum, although this obviously depends on the precise features of the model. A fourth difficulty is more theoretical than experimental. The action-reaction principle implies that if a mass $m_{1}$ feels a force $\left(\sqrt{G m_{2} a_{0}}\right) m_{1} / r$ caused by a second mass $m_{2}$, in the MOND regime, then the latter must feel the opposite force and thereby undergo an acceleration $\left(\sqrt{G a_{0} / m_{2}}\right) m_{1} / r$. Since this expression diverges as $m_{2} \rightarrow 0$, any light enough particle should thus be infinitely accelerated by distant objects feeling its MOND potential. Actually, the self-energy of such particles must be properly renormalized, and it has been proven in [27] that the RAQUAL model does not suffer from any inconsistency due to the action-reaction principle. Indeed, the force felt by mass $m_{1}$ takes the MOND form $\left(\sqrt{G m_{2} a_{0}}\right) m_{1} / r$ only when $m_{2} \gg m_{1}$. The exact expression of the forces has been derived in [150], $m_{1} a_{1}=m_{2} a_{2}=\left(2 \sqrt{G a_{0}} / 3 r\right)\left[\left(m_{1}+m_{2}\right)^{3 / 2}-m_{1}^{3 / 2}-m_{2}^{3 / 2}\right]$, so that the acceleration $a_{2}=\sqrt{G m_{1} a_{0}} / r+\mathcal{O}\left(\sqrt{m_{2}}\right)$ remains finite even when $m_{2} \rightarrow 0$. Although we expect that similar arguments would apply for any field theory deriving from an action principle, it remains to explicitly prove so for new models that one may consider, for instance for the class of nonminimally coupled fields studied in Sec. $[\mathrm{V}$ above.

All these difficulties, in addition to those discussed in the present paper, underline that the construction of a consistent field theory reproducing the MOND dynamics and the right light deflection is far from being obvious. Although the considered actions depend on several free functions allowing us to fit different kinds of data, there are so many theoretical and experimental constraints that no model passes all of them at present. The conclusion of our analysis seems thus to be in favor of the dark matter paradigm. On the other hand, the Tully-Fisher law, i.e., the existence of a universal acceleration scale $a_{0}$, has not yet been derived in dark matter models. More generally, dark matter profiles seem to be tightly correlated to baryonic ones [151] (up to apparent counterexamples such as the bullet cluster [143, 144]), and this lacks any explanation in CDM whereas this is an obvious prediction of MOND. Therefore, the dark and baryonic matter clustering still needs to be understood in more detail, and the analysis of modified gravity theories remains an interesting alternative to the standard $\Lambda$ CDM paradigm.

Identifying theoretical difficulties and finding ways to solve them often provides new possible interpretations of experimental data. For instance, we saw in Sec. IIF that the RAQUAL kinetic term needed to be slightly modified for vanishingly small accelerations, in order for the field theory to remain consistent. The resulting model predicts that gravity becomes Newtonian again at very large distances (after a transition by the MOND regime), but with a much larger gravitational constant. Both the cosmological predictions and our interpretation of cosmological data would be fully changed within such a model. This may lead to a new understanding of both the "local" mass discrepancies (within galaxies and clusters) and of the cosmological dark matter. Another bonus of our study was also to provide an example of a consistent field theory reproducing the Pioneer anomaly without 
spoiling other predictions of general relativity. But although it passes theoretical and experimental constraints, it does not pass "esthetical" ones (as defined in the Introduction), i.e., it is actually tuned to account for Pioneer data. It would remain to find an underlying symmetry principle which could predict this phenomenology.

\section{Acknowledgments}

We wish to thank many colleagues for discussions about dark matter and MOND, notably L. Blanchet, T. Damour, C. Deffayet, É. Flanagan, B. Fort, A. Lue, G. Mamon, J. Moffat, Y. Mellier, M. Milgrom, R. Sanders, J.-P. Uzan and R. Woodard.

[1] C. M. Will, Theory and experiment in gravitational physics (Cambridge University Press, Cambridge, 1993).

[2] C. M. Will, Living Rev. Rel. 9, 3 (2006), http://www.livingreviews.org/lrr-2006-3, gr-qc/0510072.

[3] D. N. Spergel et al. (WMAP), Astrophys. J. Suppl. 148, 175 (2003), astro-ph/0302209.

[4] D. N. Spergel et al. (WMAP) (2006), astro-ph/0603449.

[5] J. L. Tonry et al. (Supernova Search Team), Astrophys. J. 594, 1 (2003), astro-ph/0305008.

[6] R. A. Knop et al. (Supernova Cosmology Project), Astrophys. J. 598, 102 (2003), astro$\mathrm{ph} / 0309368$.

[7] A. G. Riess et al. (Supernova Search Team), Astrophys. J. 607, 665 (2004), astro-ph/0402512.

[8] P. Astier et al. (The SNLS), Astron. Astrophys. 447, 31 (2006), astro-ph/0510447.

[9] V. C. Rubin, N. Thonnard, and J. Ford, W. K., Astrophys. J. 225, L107 (1978).

[10] J. D. Anderson et al., Phys. Rev. D65, 082004 (2002), gr-qc/0104064.

[11] C. Lämmerzahl, O. Preuss, and H. Dittus (2006), gr-qc/0604052.

[12] B. Ratra and P. J. E. Peebles, Phys. Rev. D37, 3406 (1988).

[13] R. R. Caldwell, R. Dave, and P. J. Steinhardt, Phys. Rev. Lett. 80, 1582 (1998), astro$\mathrm{ph} / 9708069$.

[14] I. Zlatev, L.-M. Wang, and P. J. Steinhardt, Phys. Rev. Lett. 82, 896 (1999), astro$\mathrm{ph} / 9807002$.

[15] C. Boehm, P. Fayet, and J. Silk, Phys. Rev. D69, 101302 (2004), hep-ph/0311143.

[16] E. Hayashi et al., Mon. Not. Roy. Astron. Soc. 355, 794 (2004).

[17] M. Milgrom, Astrophys. J. 270, 365 (1983).

[18] R. H. Sanders and S. S. McGaugh, Ann. Rev. Astron. Astrophys. 40, 263 (2002), astro$\mathrm{ph} / 0204521$.

[19] R. B. Tully and J. R. Fisher, Astron. Astrophys. 54, 661 (1977).

[20] G. R. Dvali, G. Gabadadze, and M. Porrati, Phys. Lett. B485, 208 (2000), hep-th/0005016.

[21] G. R. Dvali and G. Gabadadze, Phys. Rev. D63, 065007 (2001), hep-th/0008054.

[22] C. Deffayet, Phys. Lett. B502, 199 (2001), hep-th/0010186.

[23] C. Deffayet, G. R. Dvali, and G. Gabadadze, Phys. Rev. D65, 044023 (2002), astro$\mathrm{ph} / 0105068$.

[24] C. Armendáriz-Picón, T. Damour, and V. F. Mukhanov, Phys. Lett. B458, 209 (1999), hep-th/9904075. 
[25] T. Chiba, T. Okabe, and M. Yamaguchi, Phys. Rev. D62, 023511 (2000), astro-ph/9912463.

[26] C. Armendáriz-Picón, V. F. Mukhanov, and P. J. Steinhardt, Phys. Rev. D63, 103510 (2001), astro-ph/0006373.

[27] J. Bekenstein and M. Milgrom, Astrophys. J. 286, 7 (1984).

[28] J. D. Bekenstein, Phys. Rev. D48, 3641 (1993), gr-qc/9211017.

[29] J. D. Bekenstein and R. H. Sanders, Astrophys. J. 429, 480 (1994), astro-ph/9311062.

[30] J. D. Bekenstein, Phys. Rev. D70, 083509 (2004), astro-ph/0403694.

[31] J. D. Bekenstein, PoS JHW2004, 012 (2005), astro-ph/0412652.

[32] R. H. Sanders, Astrophys. J. 480, 492 (1997), astro-ph/9612099.

[33] R. H. Sanders, Mon. Not. Roy. Astron. Soc. 363, 459 (2005), astro-ph/0502222.

[34] M. A. Clayton (2001), gr-qc/0104103.

[35] G. D. Moore and A. E. Nelson, JHEP 109, 023 (2001), hep-ph/0106220.

[36] J. W. Elliott, G. D. Moore, and H. Stoica, JHEP 08, 066 (2005), hep-ph/0505211.

[37] S. Baessler et al., Phys. Rev. Lett. 83, 003585 (1999).

[38] J. G. Williams, S. G. Turyshev, and D. H. Boggs, Phys. Rev. Lett. 93, 261101 (2004), gr-qc/0411113.

[39] C. W. Misner, K. S. Thorne, and J. A. Wheeler, Gravitation (Freeman, San Francisco, 1973).

[40] M. Milgrom, Ann. Phys. 229, 384 (1994), astro-ph/9303012.

[41] M. Milgrom, Phys. Lett. A253, 273 (1999), astro-ph/9805346.

[42] M. E. Soussa and R. P. Woodard, Class. Quant. Grav. 20, 2737 (2003), astro-ph/0302030.

[43] L. Blanchet (2006), astro-ph/0605637.

[44] L. Blanchet (2006), gr-qc/0609121.

[45] F. Piazza and C. Marinoni, Phys. Rev. Lett. 91, 141301 (2003), hep-ph/0304228.

[46] T. Damour, G. W. Gibbons, and C. Gundlach, Phys. Rev. Lett. 64, 123 (1990).

[47] T. Damour and C. Gundlach, Phys. Rev. D43, 3873 (1991).

[48] J. A. Casas, J. Garcia-Bellido, and M. Quiros, Class. Quant. Grav. 9, 1371 (1992), hep$\mathrm{ph} / 9204213$.

[49] G. W. Anderson and S. M. Carroll (1997), astro-ph/9711288.

[50] L. Amendola, Phys. Rev. D62, 043511 (2000), astro-ph/9908023.

[51] D. Comelli, M. Pietroni, and A. Riotto, Phys. Lett. B571, 115 (2003), hep-ph/0302080.

[52] G. R. Farrar and P. J. E. Peebles, Astrophys. J. 604, 1 (2004), astro-ph/0307316.

[53] L. Amendola, G. Camargo Campos, and R. Rosenfeld, Phys. Rev. D75, 083506 (2007), astro-ph/0610806.

[54] M. Cadoni, Gen. Rel. Grav. 36, 2681 (2004), gr-qc/0312054.

[55] Y. Sobouti (2006), astro-ph/0603302.

[56] S. Capozziello, V. F. Cardone, and A. Troisi, Mon. Not. Roy. Astron. Soc. 375, 1423 (2007), astro-ph/0603522.

[57] W. F. Kao (2005), gr-qc/0512062.

[58] A. Einstein, Sitz. Preuss. Akad. Wiss. p. 414 (1925).

[59] A. Einstein and E. G. Straus, Ann. Math. 47, 731 (1946).

[60] J. W. Moffat, Phys. Rev. D19, 3554 (1979).

[61] T. Damour, S. Deser, and J. G. McCarthy, Phys. Rev. D47, 1541 (1993), gr-qc/9207003.

[62] J. W. Moffat (2004), astro-ph/0403266.

[63] J. W. Moffat (2004), gr-qc/0404076.

[64] J. W. Moffat, JCAP 0505, 003 (2005), astro-ph/0412195.

[65] J. W. Moffat, JCAP 0603, 004 (2006), gr-qc/0506021. 
[66] J. R. Brownstein and J. W. Moffat, Astrophys. J. 636, 721 (2006), astro-ph/0506370.

[67] G. 't Hooft and M. J. G. Veltman, Annales Inst. H. Poincaré, Phys. Théor., A20, 69 (1974).

[68] K. S. Stelle, Phys. Rev. D16, 953 (1977).

[69] R. P. Woodard (2006), astro-ph/0601672.

[70] A. Hindawi, B. A. Ovrut, and D. Waldram, Phys. Rev. D53, 5597 (1996), hep-th/9509147.

[71] E. T. Tomboulis, Phys. Lett. B389, 225 (1996), hep-th/9601082.

[72] I. Navarro and K. Van Acoleyen, JCAP 0603, 008 (2006), gr-qc/0511045.

[73] G. Cognola, E. Elizalde, S. Nojiri, S. D. Odintsov, and S. Zerbini, Phys. Rev. D73, 084007 (2006), hep-th/0601008.

[74] M. Ostrogradski, Mem. Ac. St. Petersbourg Rev. VI 4, 385 (1850).

[75] J. Z. Simon, Phys. Rev. D41, 3720 (1990).

[76] P. W. Higgs, Nuovo Cimento 11, 816 (1959).

[77] G. Bicknell, J. Phys. A 7, 1061 (1974).

[78] P. Teyssandier and P. Tourrenc, J. Math. Phys. 24, 2793 (1983).

[79] B. Whitt, Phys. Lett. B145, 176 (1984).

[80] L. Bel and H. Sirousse Zia, Phys. Rev. D 32, 3128 (1985).

[81] H.-J. Schmidt, Astron. Nachr. 308, 183 (1987), gr-qc/0106035.

[82] D. Wands, Class. Quant. Grav. 11, 269 (1994), gr-qc/9307034.

[83] É. É. Flanagan, Phys. Rev. Lett. 92, 071101 (2004), astro-ph/0308111.

[84] É. É. Flanagan, Class. Quant. Grav. 21, 417 (2003), gr-qc/0309015.

[85] C. Brans and R. H. Dicke, Phys. Rev. 124, 925 (1961).

[86] P. Jordan, Z. Phys. 157, 112 (1959).

[87] M. Fierz, Helv. Phys. Acta 29, 128 (1956).

[88] B. Bertotti, L. Iess, and P. Tortora, Nature 425, 374 (2003).

[89] T. Damour and G. Esposito-Farèse, Class. Quant. Grav. 9, 2093 (1992).

[90] T. Damour and G. Esposito-Farèse, Phys. Rev. Lett. 70, 2220 (1993).

[91] T. Damour and G. Esposito-Farèse, Phys. Rev. D54, 1474 (1996), gr-qc/9602056.

[92] T. Damour and G. Esposito-Farèse, Phys. Rev. D58, 042001 (1998), gr-qc/9803031.

[93] S. Gottlöber, H. J. Schmidt, and A. A. Starobinsky, Class. Quant. Grav. 7, 893 (1990).

[94] J. D. Bekenstein, Phys. Lett. B202, 497 (1988).

[95] R. H. Sanders, Mon. Not. Roy. Astron. Soc. 235, 105 (1988).

[96] D. G. Boulware and S. Deser, Phys. Lett. B175, 409 (1986).

[97] G. Esposito-Farèse (2003), in Proceedings of the XXXVIIIth Rencontres de Moriond, March 2003, Thê' Giói Publishers (Vietnam), p. 427, gr-qc/0306018; E. Semboloni, laurea thesis (2002), unpublished.

[98] G. Esposito-Farèse, AIP Conf. Proc. 736, 35 (2004), gr-qc/0409081.

[99] L. Amendola, C. Charmousis, and S. C. Davis, JCAP 0612, 020 (2006), hep-th/0506137.

[100] L. Amendola, C. Charmousis, and S. C. Davis (2007), arXiv:0704.0175 [astro-ph].

[101] Y. Aharonov, A. Komar, and L. Susskind, Phys. Rev. 182, 1400 (1969).

[102] A. Adams, N. Arkani-Hamed, S. Dubovsky, A. Nicolis, and R. Rattazzi, JHEP 10, 014 (2006), hep-th/0602178.

[103] J.-P. Bruneton, Phys. Rev. D75, 085013 (2007), gr-qc/0607055.

[104] G. Ellis, R. Maartens, and M. A. H. MacCallum (2007), gr-qc/0703121.

[105] G. Gentile, P. Salucci, U. Klein, and G. L. Granato, Mon. Not. Roy. Astron. Soc. 375, 199 (2007), astro-ph/0611355.

[106] T. Jacobson and D. Mattingly, Phys. Rev. D64, 024028 (2001), gr-qc/0007031. 
[107] C. Eling, T. Jacobson, and D. Mattingly (2004), gr-qc/0410001.

[108] S. M. Carroll and E. A. Lim, Phys. Rev. D70, 123525 (2004), hep-th/0407149.

[109] C. Talmadge, J. P. Berthias, R. W. Hellings, and E. M. Standish, Phys. Rev. Lett. 61, 1159 (1988).

[110] L. Lindegren and M. A. C. Perryman, Astron. Astrophys. Suppl. Ser. 116, 579 (1996), see also www.rssd.esa.int/gaia/.

[111] S. G. Turyshev, M. Shao, and J. Nordtvedt, Kenneth, Class. Quant. Grav. 21, 2773 (2004), gr-qc/0311020.

[112] S. G. Turyshev, M. Shao, and J. Nordtvedt, Kenneth L., Int. J. Mod. Phys. D13, 2035 (2004), gr-qc/0410044.

[113] Y. Fujii and M. Sasaki, Phys. Rev. D75, 064028 (2007), astro-ph/0608508.

[114] B. Fort and Y. Mellier, Astron. Astrophys. Rev. 5, 239 (1994).

[115] Y. Mellier, Ann. Rev. Astron. Astrophys. 37, 127 (1999), astro-ph/9812172.

[116] M. Bartelmann and P. Schneider, Phys. Rept. 340, 291 (2001), astro-ph/9912508.

[117] S. Weinberg, Gravitation and cosmology (John Wiley and Sons, New-York, 1972).

[118] W.-T. Ni, Phys. Rev. D7, 2880 (1973).

[119] M. A. Clayton and J. W. Moffat, Phys. Lett. B477, 269 (2000), gr-qc/9910112.

[120] B. A. Bassett, S. Liberati, C. Molina-Paris, and M. Visser, Phys. Rev. D62, 103518 (2000), astro-ph/0001441.

[121] B. Z. Foster and T. Jacobson, Phys. Rev. D73, 064015 (2006), gr-qc/0509083.

[122] T. Damour and G. Esposito-Farèse, Phys. Rev. D49, 1693 (1994), gr-qc/9311034.

[123] T. Jacobson and D. Mattingly, Phys. Rev. D70, 024003 (2004), gr-qc/0402005.

[124] G. Esposito-Farèse (2005), in Proceedings of the tenth Marcel Grossmann Meeting, July 2003, World Scientific, p. 647, gr-qc/0402007.

[125] T. Damour (2007), arXiv:0704.0749 [gr-qc].

[126] T. Damour and G. Esposito-Farèse (2007), in preparation.

[127] D. Eardley, Astrophys. J. 196, L59 (1975).

[128] C. Will and D. Eardley, Astrophys. J. 212, L91 (1977).

[129] K. Nordtvedt, Phys. Rev. D49, 5165 (1994).

[130] W. D. Goldberger and I. Z. Rothstein, Phys. Rev. D73, 104029 (2006), hep-th/0409156.

[131] E. O. Kahya and R. P. Woodard (2007), arXiv:0705.0153 [astro-ph].

[132] M. M. Nieto and S. G. Turyshev, Class. Quant. Grav. 21, 4005 (2004), gr-qc/0308017.

[133] S. G. Turyshev, V. T. Toth, L. R. Kellogg, E. L. Lau, and K. J. Lee, Int. J. Mod. Phys. D15, 1 (2006), gr-qc/0512121.

[134] M.-T. Jaekel and S. Reynaud, Class. Quant. Grav. 22, 2135 (2005), gr-qc/0502007.

[135] M.-T. Jaekel and S. Reynaud, Class. Quant. Grav. 23, 777 (2006), gr-qc/0510068.

[136] M.-T. Jaekel and S. Reynaud, Class. Quant. Grav. 23, 7561 (2006), gr-qc/0610155.

[137] A. Eddington, The Mathematical Theory of Relativity (Cambridge University Press, London, 1923).

[138] L. I. Schiff, Am. J. Phys. 28, 340 (1960).

[139] R. Baierlein, Phys. Rev. 162, 1275 (1967).

[140] K. Nordtvedt, Phys. Rev. 169, 1017 (1968).

[141] C. M. Will, Astrophys. J. 163, 611 (1971).

[142] C. M. Will and K. Nordtvedt, Astrophys. J. 177, 757 (1972).

[143] D. Clowe, A. Gonzalez, and M. Markevitch, Astrophys. J. 604, 596 (2004), astro-ph/0312273.

[144] M. J. Jee et al. (2007), arXiv:0705.2171 [astro-ph]. 
[145] G. W. Angus, B. Famaey, and H. Zhao, Mon. Not. Roy. Astron. Soc. 371, 138 (2006), astro-ph/0606216.

[146] G. W. Angus, H. Shan, H. Zhao, and B. Famaey, Astrophys. J. 654, L13 (2007), astroph/0609125.

[147] J. R. Brownstein and J. W. Moffat (2007), astro-ph/0702146.

[148] G. W. Angus and S. S. McGaugh (2007), arXiv:0704.0381 [astro-ph].

[149] C. Skordis, D. F. Mota, P. G. Ferreira, and C. Boehm, Phys. Rev. Lett. 96, 011301 (2006), astro-ph/0505519.

[150] M. Milgrom, Phys. Rev. E56, 1148 (1997), gr-qc/9705003.

[151] S. McGaugh, Phys. Rev. Lett. 95, 171302 (2005), astro-ph/0509305. 